\title{
The skin environment controls local dendritic cell differentiation and function through innate IL-13
}

Johannes U Mayer ${ }^{1 *}$, Olivier Lamiable ${ }^{1 *}$, Kerry L Hilligan ${ }^{1,2}$, Jodie S Chandler ${ }^{1}$, Samuel I Old ${ }^{1}$, David A Eccles ${ }^{1}$, Jianping Yang ${ }^{1}$, Greta R Webb ${ }^{1}$, Rita G Domingues ${ }^{3}$, Luis Munoz-Erazo ${ }^{1}$, Kirsty A Wakelin ${ }^{1}$, Evelyn J Hyde ${ }^{1}$, Shiau-Choot Tang ${ }^{1}$, Sally C Chappell ${ }^{1}$, Charles R Mackay ${ }^{4}$, Frank Brombacher ${ }^{5}$, Alan Sher ${ }^{2}$, Roxane Tussiwand ${ }^{6,7}$, Lisa M Connor ${ }^{1,8}$, Dragana Jankovic ${ }^{9}$, Matthew R Hepworth ${ }^{3}$, Graham Le Gros ${ }^{1}$, Franca Ronchese ${ }^{1, * *}$

${ }^{1}$ Malaghan Institute of Medical Research, Wellington 6012, New Zealand.

${ }^{2}$ Immunobiology Section, Laboratory of Parasitic Diseases, National Institute of Allergy and Infectious Diseases, National Institutes of Health, Bethesda 20892 MD, USA.

${ }^{3}$ Lydia Becker Institute of Immunology and Inflammation, Manchester Collaborative Centre for Inflammation Research, Faculty of Biology, Medicine and Health, University of Manchester, Manchester Academic Health Science Centre, Manchester, UK.

${ }^{4}$ Infection and Immunity Program, Monash Biomedicine Discovery Institute, Monash University, Melbourne, Australia.

${ }^{5}$ International Centre for Genetic Engineering and Biotechnology (ICGEB), Cape Town component \& Institute of Infectious Diseases and Molecular Medicine (IDM), Division of Immunology, Health Science Faculty, University of Cape Town, South Africa.

${ }^{6}$ Department of Biomedicine, University of Basel, 4058 Basel, Switzerland

${ }^{7}$ Immune Regulation Unit, National Institute of Dental and Craniofacial Research, National Institutes of Health, Bethesda 20892 MD, USA.

8 Present address: School of Biological Sciences, Victoria University of Wellington, Wellington 6012, New Zealand.

${ }^{9}$ Immunoparasitology Unit, Laboratory of Parasitic Diseases, National Institute of Allergy and Infectious Diseases, National Institutes of Health, Bethesda 20892 MD, USA.

* These authors contributed equally_** Lead contact.

Correspondence: Dr Franca Ronchese fronchese@malaghan.org.nz

Keywords: Dendritic cells; Skin; IL-13; STAT6; ILC; T cell responses; Immune regulation. 


\section{ABSTRACT}

The signals driving the adaptation of type-2 dendritic cells (DC2s) to diverse peripheral environments are not well understood. We show that the development of CD11 blow migratory DC2s, a DC2 population unique to the dermis, requires STAT6- and KLF4dependent IL-13 signaling, whereas DC2s in lung and small intestine are STAT6-independent. Dermal IL-13 is mostly derived from innate lymphoid cells expressing a resting ICOS+ KLRG1ST2- phenotype. Analysis of public datasets indicates that human skin DC2s also express an IL-4/IL-13 gene signature compared to blood or spleen, suggesting a similar developmental pathway in mice and humans. In the absence of IL-13 signaling, dermal DC2s are stable in number but remain $C D 11 b^{\text {hi }}$ and show defective activation in response to allergen with diminished ability to support IL-4+ GATA3+ Th development, whereas anti-fungal IL-17+ ROR $\gamma \mathrm{t}+$ responses are increased. Thus, steady-state IL-13 fosters a non-inflammatory and pro-allergic environment in healthy skin via conditioning of local DC2s. 


\section{INTRODUCTION}

DCs play a crucial role in informing the adaptive immune system about infectious, innocuous or self antigens and directing $T$ cell responses towards effector activation, ignorance or tolerance (Eisenbarth, 2019). Two developmentally distinct lineages of DCs termed 'type 1' and 'type 2' (DC1 and DC2, respectively) were initially described in the bone marrow (Schlitzer et al., 2015) and are now identified in all tissues in humans and mice (Guilliams et al., 2016). In general terms, DC1s are characterised by the expression of XCR1 and predominantly interact with CD8 T cells to cross-present cell-associated antigens and initiate immune responses against intracellular pathogens. DC2s, on the other hand, interact with CD4 T cells to promote T helper (Th) and T regulatory cell responses (Dudziak et al., 2007; Hilligan and Ronchese, 2020). Whilst these broad DC functions are relevant to, and conserved in, all areas of the body, the specific requirements of each tissue may also demand that these properties are appropriately modulated to promote homeostasis and optimal tissue function.

DC2s share several common characteristics that differentiate them from DC1s, including the expression of CD172a/Sirp $\alpha$ and being mostly depleted from the lymph nodes (LNs) of full or conditional IRF4 knockout (KO) mice (Bajana et al., 2016; Persson et al., 2013; Schlitzer et al., 2013) and humans (Guerin et al., 2018), thus validating their classification into different developmental lineages (Guilliams et al., 2014). Nonetheless, tissue DC2s are also considerably heterogeneous in phenotype, a feature that is especially notable in the migratory DC2 population that migrates from non-lymphoid tissues to secondary lymphoid organs (Alcantara-Hernandez et al., 2017; Heidkamp et al., 2016). Migratory DC2s from different tissues express unique markers that are largely conserved in mice and humans (Guilliams et al., 2016), suggesting that such heterogeneity may be acquired in response to the diverse homeostatic and functional requirements of each tissue (Sichien et al., 2017). Evidence that specific tissue-derived signals can influence the phenotype and function of local DC2s has been reported for the small intestine (SI), where retinoic acid and TGF $\beta$ have been identified as unique factors driving the development and functional maturation of intestinal $C D 103^{+}$DC2s (Bain et al., 2017; Zeng et al., 2016). It remains unclear whether and which tissue-derived signals guide DC2 development at other sites.

Unlike the intestinal tract and airway, which are specialized for nutrient absorption and gas exchange and as such must tolerate the resulting exposure to many antigenic stimuli, the skin's function is to block exchange thereby reducing water and nutrient loss to maintain the body's internal environment. The skin's barrier function is collectively carried out by several layers of tightly packed epithelial cells and sebaceous gland secretions forming an impermeable barrier. In addition, local immune cells and neural circuits perceive external threats via sensing of tissue damage, IgE-dependent mast cell activation and IL-31dependent itch responses to control biting insects, remove ectoparasites, and limit contact 
with plant irritants (Bagci and Ruzicka, 2018; Olomski et al., 2020; Perner et al., 2020; Prussin and Metcalfe, 2003). Concomitantly, the skin's continued exposure to pathogens and commensals must be managed to ensure that local invasion is prevented, tissue is repaired, and barrier function is quickly restored (Harrison et al., 2019). It is plausible that these specialized skin functions are supported by unique skin networks that are distinct from those in tissues such as lung and gut (Ronchese et al., 2020).

The skin harbours four subsets of migratory dendritic and dendritic-like cells each expressing distinct transcriptomic signatures (Miller et al., 2012). Langerhans cells represent a unique epidermal DC-like population which is developmentally distinct from conventional DCs and specialises in tolerance induction (Shklovskaya et al., 2011). Dermal DCs include DC1 and DC2 populations, with the latter being further divided into CD11 bi $\mathrm{b}^{\text {hi }}$ and $11 \mathrm{~b}^{\text {low }}$ expressing DC2s (Henri et al., 2010). DC2s represent the most abundant dermal DC population in both mice and humans (Henri et al., 2010; Segura et al., 2012). They are mostly found in the vicinity of hair follicles but also in the deeper layer of the skin, where they form specialised niches with other immune cells including innate lymphoid cells (ILCS) (Dahlgren et al., 2019). Dermal CD11 b ${ }^{\text {hi }}$ DC2s have been shown to induce T regulatory and anti-microbial immune responses (Naik et al., 2015; Tordesillas et al., 2018), whereas CD11b $b^{\text {low }}$ DC2s are highly responsive to Thymic Stromal Lymphopoietin (TSLP) and have been associated with driving type 2 immunity and allergic responses (Ochiai et al., 2014; Tussiwand et al., 2015). As the CD11b low DC2 phenotype is unique to skin, their study may provide insight into the signals shaping the skin immune environment.

Here we report that the development of $C D 11 b^{\text {low }}$ dermal $D C 2 s$ requires canonical signalling through the type II IL-4 and IL-13 receptor and signal transducer and activator of transcription 6 (STAT6), and is associated with the steady-state expression of Interleukin-13 (IL-13) by ICOS ${ }^{+}$KLRG1- ST2- ILC2s in naïve skin. No similar steady-state IL-13 expression and signaling in DC2s were observed in naïve lung or SI. In humans, IL-13-regulated genes were preferentially enriched in DC2s from healthy skin, but not lung or blood, suggesting a cytokine environment similar to murine skin. IL-13 signaling in dermal DCs promoted optimal IL-4 responses to a helminth allergen but was inhibitory to IL-17A responses to a fungal pathogen. Thus, our work identifies a skin-specific, IL-13-mediated signalling circuit between an ILC subset and DC2 precursors which is required for the development of the distinctive CD11b ${ }^{\text {low }}$ skin DC2 subset and establishes an anti-inflammatory, pro-Th2 environment in skin. 


\section{RESULTS}

\section{The transcriptomic signature of CD11 $b^{\text {low }}$ migratory DC2s in skin-draining $L N(d L N)$ is enriched in STAT6-binding sites}

To identify factors controlling the tissue-specific programming of DC2s, we developed a flow cytometric staining panel to define distinct migratory DC2 subsets in the dLNs of the skin, lung and SI. Monocytes, macrophages and neutrophils were excluded through their respective expression of Ly6C, CD64 and Ly6G, migratory DCs were identified by their expression of intermediate $\mathrm{CD} 11 \mathrm{c}$ and high $\mathrm{MHCll}$, and DC1s, Langerhans cells and DC2s were distinguished by their respective expression of XCR1, CD326 and Sirpa (Figure S1A) (Guilliams et al., 2016). High-dimensional analysis of concatenated samples confirmed that DC2s from different $L N$ s expressed several unique surface markers with limited overlap and could be defined as CD11 bi and CD11b $b^{\text {low }}$ DC2s in the skin-dLN, CD24+ and CD24- DC2s in the lung-dLN, and CD103+ and CD103- DC2s in the SI-dLN (Figures 1A and 1B). These same phenotypes were also observed in the respective tissues of origin (Figures $\mathbf{1 C}$ and S1B), were present in male and female mice although in significantly different frequencies in skin (Figure 1D), and were observed in both BALB/C and C57BL/6 (C57) mice (Figure 1E), indicating that these surface markers defined conserved tissue-specific DC2 subsets under steady-state conditions. To confirm that all of these subsets were genuine bona-fide DC2s, we assessed $/ r f 4^{f / f} \mathrm{CD} 11 \mathrm{c}$-Cre mice in which DC2 numbers in each of the tissue-dLNs are very low (Bajana et al., 2016; Mayer et al., 2017; Persson et al., 2013; Schlitzer et al., 2013). As expected, DC2 subsets in the skin, lung and SI-dLN of Irf $4^{f / f} \mathrm{CD} 11 \mathrm{c}$-Cre mice were all significantly reduced compared to $\operatorname{lrf} 4^{\mathrm{f} / \mathrm{f}}$ mice (Figure S1C).

To compare the gene expression profile of each tissue-specific DC2 subset, we performed bulk RNA-sequencing of sorted migratory DC2s from the skin, lung and SI-dLN of C57 mice. A Principal Component Analysis (PCA) of all expressed genes revealed three main clusters on PC1 vs. PC2 (Figure 1F). Cluster I included SI-dLN CD103- DC2s and CD24- DC2s from the lung-dLN, cluster II included SI-dLN CD103+ DC2, and cluster III included both skin-dLN DC2 subsets as well as CD24+ DC2 from the lung-dLN. When PC3 was taken into account, cluster III could be further resolved into a heterogeneous cluster of skin-dLN CD11 b ${ }^{\text {hi }}$ DC2s and lung-dLN CD24+ DC2s (cluster III-a) and a separate cluster of skin-dLN CD11b low DC2s (cluster III-b). Using DESeq2 we defined a gene signature for each cluster, which included protein-coding genes that were expressed at least 2-fold higher in that cluster compared to each of the other clusters with a $p$-value $<0.05$. Based on this analysis, 101 genes were associated with cluster I, 86 with cluster II, 37 with cluster III-a, and 78 with cluster III-b (Figure 1G and Table S1).

To determine whether differential gene expression in these different DC2 clusters might be controlled by unique transcription factors, we examined the promoter sequences of the 
cluster-specific signature genes for enriched transcription factor binding sites (TFBS). The majority of the TFBS were shared between multiple clusters, while 2 were uniquely enriched in cluster I, 1 in cluster II and 9 in cluster III-b (Figure $\mathbf{1 H}$ and Table S2). No TFBS were unique to cluster III-b, possibly suggesting that these DCs may represent a 'default' developmental pathway. Of the 9 TFBS enriched in cluster III-b, two were for transcription factors expressed at low levels in DC2 (FOXO4, POU6F1), six were linked to interferon responses, DC2 development or T cell priming (IRF1, IRF2, IRF4, NFATc1, NFATc3, HMGA1), and one was for STAT6, a transcription factor that has no described role in the context of DC2 biology at steady state. Given that STAT6 is essential for signaling by the Th2 cytokines IL-4 and IL-13 (Ansel et al., 2006), the enrichment of STAT6-binding sites in CD11 b ${ }^{\text {low }}$ DC2 signature genes suggested that these DC had been uniquely exposed to IL-4 and/or IL-13 in vivo.

\section{The development of dermal CD11 $b^{\text {low }}$ DC2s is impaired in STAT6-KO mice}

To determine whether STAT6 signalling played a role in DC2 development by affecting DC2 precursors, we first identified the $\mathrm{Lin}^{-} \mathrm{CD} 11 \mathrm{C}^{+} \mathrm{MHCII}^{-} \mathrm{CD} 135^{+}$preDC population in the bone marrow (BM) and ear skin of C57 and STAT6-KO mice (Figure S2A). Within preDCs, the Ly6C SiglecH ${ }^{-}$preDC2 population (Schlitzer et al., 2015) was present at steady state in both mouse strains in similar frequencies (Figures 2A and S2A), suggesting that STAT6 signalling did not affect preDC2 development or egress from the BM. In addition, skin preDC2s expressed similarly high levels of CD11b (Figure 2A), indicating that the downregulation of CD11b in dermal CD11b ${ }^{\text {low }} \mathrm{DC} 2 \mathrm{~s}$ was occurring during maturation in the skin.

Analysis of mature DC2 subsets in skin, lung and SI revealed that STAT6-KO mice exhibited a significant reduction in CD11 bow $D C 2 s$ and a reciprocal increase in CD11 b $b^{\text {hi }}$ DC2s in ear skin, while the distribution of CD24 positive and negative DC2s in the lung and CD103 positive and negative DC2s in the SI were unchanged (Figure 2B and 2C). Within the skin, defective STAT6 signalling did not affect the frequency of DC1s, Langerhans cells (Figure 2C) or other myeloid cell populations (Figure S2B), pointing towards a unique requirement for STAT6 in $C D 11 b^{\text {low }}$ DC2 development. Migratory DCs in the dLNs were similarly affected, with CD11 b ${ }^{\text {low }}$ DC2s in the skin-dLN significantly reduced in STAT6-KO mice compared to C57, whereas DC2 subsets in lung or SI-dLNs, and DC1 and Langerhans cells within the skin-dLN, were present in comparable proportions (Figures 2D and 2E). The findings in ear skin-dLN also applied to LNs draining other areas of the skin (Figure $\mathbf{2 F}$ ), indicating that CD11b low DC2s follow a similar developmental program irrespective of skin region.

To explore the impact of STAT6-dependent signaling on DC2s in further depth, we carried out a transcriptomic characterization of DC2 subsets from the skin, lung and SI-dLNs of C57 and STAT6-KO mice. Analysis of bulk RNAseq data revealed a number of differences between skin-dLN DC2 populations in STAT6-KO and C57 mice. 171 genes were upregulated and 74 downregulated in CD11b ${ }^{\text {low }}$ STAT6-KO DC2s compared to C57 (Figure 2G and Table 
S3); amongst the downregulated transcripts were $\mathrm{Ccl} 17$ and $\mathrm{Ccl} / 5$, two chemokines involved in functional interactions between DCs and T cells (Griffith et al., 2014), whereas transcripts for the DC lineage markers Cd24a and Esam were upregulated. In CD11b ${ }^{\text {hi }}$ DC2s, 386 genes were upregulated and 74 downregulated (Figure 2G), with many of the upregulated genes involved in transcription, motility and immune system processes. Transcripts for Aldh1a2 which encodes Retinaldehyde Dehydrogenase 2, or RALDH2, were notably downregulated, while expression of $\mathrm{CCl} 17$ was not significantly changed in this subset. Only about $10 \%$ of the differentially expressed genes in $C D 11 b^{\text {hi }} D C 2 s$ were similarly regulated in the $C D 11 b^{\text {low }}$ population (Figure S2C). In line with these observations, a PCA comparing all expressed genes in skin-dLN DC2s from C57 and STAT6-KO mice showed that the separation between the two DC2 subsets was maintained across PC1, suggesting that STAT6-KO CD11 $b^{\text {hi }}$ and CD11b low DC2s both maintained their transcriptional identity (Figure S2D), with the separation between C57 and STAT6-KO DC2s becoming apparent along PC2 for the CD11 $b^{\text {hi }}$ population and PC3 for the CD11b ${ }^{\text {lo }}$. In contrast to skin-dLN DC2s, lung- and SI-dLN DC2s were not strongly affected by STAT6 deficiency. In the lung-dLN, only 6 and 3 genes were differentially expressed in CD24+ and CD24- DC2s from STAT6-KO vs. C57 mice, respectively. In the SI-dLN, there were 56 genes differentially expressed in CD103+ DC2s, mostly with high $P$ values, and 5 genes in CD103- DC2s (Figure 2G). PCAs comparing lung- or SI-dLN DC2s from C57 mice to their STAT6-KO counterparts also confirmed the overall similarity of these DC2 subsets in STAT6-KO vs. C57 mice (Figure S2D). Thus, STAT6 controls expression of a large number of genes in skin-derived $C D 11 b^{\text {hi }}$ and $C D 11 b^{\text {low }} D C 2 s$ under steady-state conditions.

Together, these data show that STAT6 signalling is required for the optimal development of dermal CD11b low DC2 in skin, while preDC2s and DC2 subsets in lung and SI and their respective dLNs are not affected.

\section{ILC-derived IL-13 is necessary and sufficient for CD11 $b^{\text {low }}$ DC2 development in vivo}

To establish whether STAT6 signalling is intrinsically required for CD11b bow DC2 development, we generated mixed wild-type and STAT6-KO BM chimeras in STAT6sufficient hosts. Analysis of the skin and skin-dLNs revealed that wild-type-derived DC2s developed into CD11 $b^{\text {hi }}$ and CD11 $b^{\text {low }}$ DC2s, whereas STAT6-KO-derived DC2s were mostly CD11 $b^{\text {hi }}$ (Figures 3A and S3A), illustrating the essential intrinsic role of STAT6 for CD11b $b^{\text {low }}$ development.

The canonical activation of STAT6 is mediated by the cytokines IL-4 or IL-13 that respectively signal through the IL-4R $\alpha /$ common- $\gamma(\gamma \mathrm{c})$ chain type I IL-4 receptor or the IL-4R $\alpha /$ IL-13R $\alpha 1$ type II IL-4 and IL-13 receptor (Wills-Karp and Finkelman, 2008), although alternative modes of STAT6 activation have also been reported (Chen et al., 2011). To address whether IL-4 or 
IL-13 signalling were responsible for STAT6 activation in dermal DC2s, we assessed IL-4-KO, IL-13-KO (Figure S3B), IL-4R $\alpha-K O$ and IL-13R $\alpha 1-K O$ mice. We observed that IL-4-KO mice displayed a normal distribution of CD11 $b^{\text {hi }}$ and CD11 bow $D C 2 s$ in the skin and minimal alterations in skin-dLN, whereas IL-13-KO mice demonstrated a pronounced reduction in CD11b low DC2s that was comparable to the level observed in STAT6-KO mice (Figures 3B, C and S3C, D). CD11 b low DC2s were also greatly reduced in the skin and skin-dLN of IL-4R $\alpha-K O$ and IL-13R $\alpha 1-K O$ mice, indicating that IL-13 signalling via the IL-4R $\alpha / I L-13 R \alpha 1$ type II receptor leads to the activation of STAT6 and is essential for the full development of CD11 b low DC2s in the skin. Transcripts for $/ 12 \mathrm{rg}$, $/ 14 \mathrm{ra}, \| 13 \mathrm{ra1}$ and other members of the IL13 signalling pathway (McCormick and Heller, 2015) were expressed at similar levels by all DC2 subsets from the lung, SI and skin-dLN (Figure 3D), and IL-4R $\alpha$ and IL-13R $\alpha 1$ protein expression was low but detectable by flow cytometry at varying levels in all DC2 populations (Figure 3E). However, defects in STAT6 signalling only affected DC2 development in the skin and skin-dLNs, suggesting that local IL-13 production rather than differential receptor expression was driving the development of CD11 b low $D C 2 s$ at steady state. To establish whether this was the case, we treated STAT6-KO and IL-13-KO mice with recombinant IL-13 fusion protein (IL-13fp) over 4 days. Injection of IL-13fp did not affect the proportion of CD11 bow and CD11b $b^{\text {hi }}$ DC2s in STAT6-KO mice, but rescued the development of CD11b low DC2s in IL-13-KO mice to proportions similar to those in C57 mice (Figure 3F). Thus, IL-13 is both necessary and sufficient to drive the development of dermal CD11b low $D C 2 s$ via the activation of STAT6 under steady-state conditions.

Previous reports have shown that skin ILC2s produce low amounts of IL-13 at steady state (Roediger et al., 2013), although no biological function for such IL-13 was identified. We undertook a thorough characterization of adaptive and innate cell populations in the skin of 4C13R mice that report I/4 expression through AmCyan and I/13 expression through DsRed (Figure S3E, F). We found that almost $30 \%$ of skin ILCs were $/ / 13-$ DsRed $^{+}$, while other innate and adaptive immune cells were less than 5\% //13-DsRed ${ }^{+}$(Figure 3G). Skin ILCs also expressed the highest level of II13-DsRed per cell compared to other popoulations, and were the most abundant /113-DsRed ${ }^{+}$cell population at steady state followed by Dendritic Epidermal T cells (DETCS) (Figure $3 \mathbf{H}$ ). Consistent with the possibility of ILCs being the source of steady-state IL-13, RAG1-KO mice showed no impairment in the development of CD11 $b^{\text {hi }}$ and CD11 blow DC2s in the skin and skin-dLN (Figure S3G), indicating that adaptive and innate-like immune cells including CD4+ T cells, DETCs, MAIT cells and NK-T cells were not essential for the steady-state production of IL-13.

Steady-state I/13-DsRed ${ }^{+}$ILCs were identified only in the skin and not in the lung or SI (Figure $3 \mathrm{I}$ ), which is consistent with our observation that the transcriptome of DC2s in lung and SI was not affected by STAT6 inactivation. //13-DsRed ${ }^{+}$and I/13-DsRed ${ }^{-}$ILCs in skin and lungs did not express //4-AmCyan and were CD44 ${ }^{\text {hi }}$ and ICOS ${ }^{+}$. Compared to lung ILCs, skin ILCs prepared and acquired under the same conditions expressed low levels of the IL-33 
receptor ST2, low CD25 (Figure 3J) and were mostly KLRG1- (Figure S3F). This observation is consistent with the described tissue adaptation and distinct expression of activating receptors on ILC2 from different tissues (Ricardo-Gonzalez et al., 2018). Together, these data are consistent with resting ILCs being the likely source of steady-state IL-13 in skin.

\section{The IL-13-dependent development of CD11 $b^{\text {low }}$ DC2 in vitro and in vivo requires KLF4 expression}

To address whether recombinant IL-13 (rIL-13) was sufficient to drive the development of CD11b bow DC2s in vitro, we generated bone marrow derived DCs (BMDCs) in the presence of FLT3L and added rIL-13 to the culture medium at different time points and concentrations. Under standard culturing conditions, B220- FLT3L BMDCs develop into XCR1 ${ }^{+}$DC1 and Sirpa ${ }^{+}$ CD11 $b^{\text {hi }}$ DC2 populations that resemble splenic DCs at steady state (Naik et al., 2007) (Figure S4A). We observed that increasing concentrations of rIL-13 (ranging from 1 to $50 \mathrm{ng} / \mathrm{ml}$ ) added on day 6 to day 9 of culture resulted in a dose-dependent development of a CD11b bw BMDC2 population (Figure S4B). A time-course experiment revealed that rIL-13 treatment was required for at least 48 hours, with the highest proportion of CD11b low $B M D C 2 s$ observed after the addition of rlL-13 over the last 72 hours (Figure S4C).

Similar to our observations in vivo, IL-13-dependent development of CD11b ${ }^{\text {low }}$ BMDC2s in vitro required the expression of IL-4R $\alpha$ and STAT6 (Figure 4A). To establish whether the similarity between in vitro and in vivo DC2s extended to genes other than CD11b, we selected a small group of genes that are differentially expressed in $C D 11 b^{\text {hi }}$ vs. CD11 $b^{\text {low }}$ DC2s in our RNA-seq data and assessed their expression in BMDCs. CD11 $b^{\text {hi }}$ DC2s from skin$\mathrm{dLN}$ and $\mathrm{CD} 11 \mathrm{~b}^{\text {hi }}$ BMDC2s both expressed higher levels of Itgax, Cc19, Abcg3 and Socs3 transcripts compared to their respective CD11b ${ }^{\text {low }}$ counterparts (Figure 4B), while Sned1, Khdc1a, Efna5 and Clmn were more highly expressed in CD11b low $D C 2 s$ in vivo and in vitro (Figure 4C). Thus, rIL-13 is sufficient to drive the in vitro development of a CD11 blow DC2 population that shares transcriptional features with in vivo dermal CD11b low $D C 2 s$.

Previous reports have shown that the development of dermal CD11b low $D C 2 s$ requires expression of the transcription factor Krüppel-like factor 4 (KLF4) (Tussiwand et al., 2015). To establish the relationship between KLF4 and IL-13 signaling in the development of CD11 $b^{\text {low }}$ DC2s, we assessed the impact of IL-13 supplementation on FLT3L BMDC cultures from either $K I f 4^{f / f}$ or $K I f 4^{f / f}$ Vav-iCre mice. Supplementation of $K I f 4^{f / f}$ cultures with rIL-13 resulted in the development of a $\mathrm{CD} 11 b^{\text {low }}$ population that was similar in frequency to the population observed in BMDC cultures from C57 mice. In contrast, rIL-13 was unable to drive the development of CD11 $b^{\text {low }}$ BMDC2s in KIf4 $4^{\mathrm{f} / \mathrm{f}}$ Vav-iCre BMDC cultures (Figure 4D), suggesting that IL-13 signaling in DC2s requires expression of KLF4. Similarly, in vivo IL-13fp treatment over 4 days failed to rescue the development of CD11b low DC2s in KIf $4^{f / f} \mathrm{Vav}$-iCre 
$\rightarrow$ C57BL/6 chimeric mice (Figure 4E), despite surface expression of IL-4R $\alpha$ and IL-13R $\alpha 1$ proteins being similar in $K I f 4^{f / f}$ and $K I f 4^{f / f}$ Vav-iCre DC2s (Figure S4D). As expected, $K I f 4^{f / f} \rightarrow C 57 B L / 6$ control chimeras displayed a normal distribution of CD11 $b^{\text {hi }}$ and CD $11 b^{\text {low }}$ DC2s in the skin-dLN. To further investigate the mechanism through which KLF4 regulates STAT6-dependent signaling, we carried out phospho-immunofluorescence experiments to compare STAT6 Y641 phosphorylation in $K I f 4^{f / f}$ and $K / f 4^{f / f}$ Vav-iCre FLT3L BMDCs exposed to rIL-13. Phosphorylated STAT6 was readily detectable in the nuclear areas of C57, Klf $4^{\mathrm{f} / \mathrm{f}}$ and KIf4 ${ }^{\mathrm{f} / \mathrm{f}}$ Vav-iCre Sirpa+ BMDCs 30 min after exposure to IL-13, but was not detected in IL4R $\alpha-K O$, STAT6-KO or untreated BMDC cultures (Figure S4E and S4F), indicating that the phosphorylation of STAT6 is functional in KLF4-deficient DC2s.

Together, these results indicate that KLF4 controls DC2 responsiveness to IL-13 in vitro and in vivo, providing a mechanism for the comparable dermal DC2 phenotype observed in mice when the KLF4 and IL-13 signaling pathways are inactivated.

\section{IL-13 signaling in DC2s is required for optimal development of IL-4+ T cells after skin immunization}

Having identified that IL-13-mediated signalling is required for CD11 b ${ }^{\text {low }}$ DC2 development in the skin, we established two models to investigate the impact of this signalling on dermal T cell immune responses. In the first model we generated mixed BM chimeric mice to examine the functional interaction of $D C$ and $T$ cell populations originating from two different donor BMs (Figure 5A). In these chimeras, $C D 4^{+} \mathrm{T}$ cells originated from $\mathrm{CD} 45.1+2+$ MHCII-KO BM and were STAT6-sufficient. In contrast, MHCII-competent DCs originated from CD45.2+ BMs and were either STAT6-KO and unable to respond to IL-13 signaling in the test chimeras, or WT and responsive to IL-13 in the control chimeras (Figures 5B and S5A-C). All chimeras were immunized intradermally with inactivated pathogens that induced either Th1, Th2 or Th17 responses (Blecher-Gonen et al., 2019; Hilligan et al., 2020) and CD4 ${ }^{+}$T cell cytokine production was assessed in the skin-dLN five days later. IFN $\gamma^{+}$responses induced by Mycobacterium smegmatis (Ms) immunization were detectable at similar levels in both C57 and STAT6-KO mixed BM chimeras (Figure $5 \mathrm{C}$ ). In contrast, IL-4+ and IL-13+ ${ }^{+}$Th cells were significantly reduced in STAT6-KO mixed BM chimeras immunized with Nippostrongylus brasiliensis L3 larvae (Nb) (Figure 5C and S5C). IL-17A ${ }^{+}$Th cell responses after Candida albicans $(\mathrm{Ca})$ immunization were trending to an increase in the STAT6-KO mixed BM chimeras, indicating that IL-13 signaling in DCs is important for the induction of Th2 cells but may be limiting the differentiation of Th17 cells (Figure 5C).

To confirm these results in a non-chimeric in vivo mouse model, we generated $/ 14 \mathrm{ra}^{\mathrm{f} / \text { z }} \mathrm{zDC}$ Cre mice in which expression of the IL-4 and IL-13 receptors is specifically deleted in DCS thereby impairing the development of CD11b low DC2s in skin and skin-dLNs (Figure 5D). 
Upon immunization with $\mathrm{Ms}$, we observed similar IFN $\gamma^{+} \mathrm{CD}^{+} \mathrm{T}$ cell responses in $/ 14 \mathrm{ra}^{\mathrm{f} /-}$ zDCCre mice compared to $/ 14 \mathrm{ra}^{\mathrm{f} /-}$, while $\mathrm{IL}-4^{+}$and $\mathrm{IL}-13^{+}$responses to $\mathrm{Nb}$ were reduced and IL$17 \mathrm{~A}^{+}$responses against $C a$ were trending to an increase (Figures 5E and S5D). Expression of the transcription factors GATA3 and ROR $\gamma \mathrm{t}$ in $\mathrm{Nb}$ or $\mathrm{Ca}$ immunized mice, respectively, reproduced the pattern observed with cytokines (Figure 5F). The pronounced reduction of Th2 responses upon selective depletion of IL-4 and IL-13 signalling in DC2s confirmed our observations in STAT6-KO mixed chimeric mice, and recapitulated the findings in $K / f 4^{f / f}$ CD11c-Cre mice (Tussiwand et al., 2015) in which impaired development of CD11b low DC2s resulted in reduced type 2 immunity after skin immunization.

To assess whether the suboptimal induction of Th2 responses in $1 / 4 \mathrm{ra}^{\mathrm{f} /-} \mathrm{zDC}$-Cre mice was due to changes in DC2 number or phenotype, we injected $/ / 4 \mathrm{ra}^{\mathrm{f} /-} \mathrm{zDC}-\mathrm{Cre}$ and $/ / 4 \mathrm{ra}^{\mathrm{f} /-}$ mice with fluorescently-labelled $\mathrm{Nb}$ ( $\mathrm{Nb}$-AF488) to identify antigen-positive cells in dLN. The total number of $\mathrm{AF} 488^{+}$cells was only moderately decreased in $/ 14 \mathrm{raf} /$ zDC-Cre mice compared to $1 / 4 \mathrm{ra}^{\mathrm{f} / \text { - }}$ (Figure 5G), but the composition of the AF488 ${ }^{+} \mathrm{DC2}$ populations (Figure $\mathbf{5 H}$ ) and the total $\mathrm{AF} 488^{+}$populations (Figure 5I) differed between the two strains. The majority of AF488 ${ }^{+}$cells in $/ / 4 \mathrm{ra}^{\mathrm{f} /-}$ mice were CD11b ${ }^{\text {low }} \mathrm{DC} 2 \mathrm{~s}$ followed by CD11 $\mathrm{b}^{\text {hi }} \mathrm{DC} 2 \mathrm{~s}$ and monocytes. Compared to $/ 14 \mathrm{ra}^{\mathrm{f} /-}$ mice, $\mathrm{AF} 488^{+}$cells in $/ / 4 \mathrm{ra}^{\mathrm{f} /-}$ zDC-Cre mice comprised a lower number of DC2s which were mainly CD11 $b^{\text {hi }}$, and an increased number of monocytes (Figure S5E-G). As monocyte depletion does not affect IL-4+ Th response to $N b$ (Hilligan et al., 2020), this increased monocyte response is unlikely to contribute to the Th response in $/ 14 \mathrm{ra}^{\mathrm{f} /-} \mathrm{zDC}$-Cre mice.

The DC signals that drive the development of Th2 responses remain undefined (Lamiable et al., 2020). We therefore examined the expression of several functional activation markers on DCs (Connor et al., 2014; Connor et al., 2017; Harris et al., 1999; Pellefigues et al., 2017). In $/ 14 \mathrm{ra}^{\mathrm{f} /-}$ mice, expression of the costimulatory molecules CD86 and PDL2 was higher on AF488 ${ }^{+}$compared to AF488- DC2s, and on CD11 b ${ }^{\text {low }}$ compared to CD11 b $\mathrm{b}^{\text {hi }} \mathrm{DC} 2 \mathrm{~s}$ (Figure $5 \mathrm{~J}$ ), a pattern of expression similar to that observed in C57 mice (Connor et al., 2017). In I/4 $\mathrm{ra}^{\mathrm{f} / \text { - }}$ zDC-Cre mice, $\mathrm{AF} 488^{+} \mathrm{CD} 11 \mathrm{~b}^{\text {hi }} \mathrm{DC} 2 \mathrm{~s}$ displayed a similar surface marker expression profile as their $\| 4 \mathrm{ra}^{\mathrm{f} / \text { - }}$ counterparts, while the number of $\mathrm{AF} 488^{+} \mathrm{CD} 11 \mathrm{~b}^{\text {low }} \mathrm{DC} 2 \mathrm{~s}$ was very low and insufficient for analysis. Expression of the Interferon response genes BST2 and Ly6A/E on CD11 $b^{\text {hi }}$ DC2s was also comparable in $/ / 4 r a^{f /-} z D C-C r e$ and $/ 14 r a^{f /-}$ mice (Figure 5J). These results suggest that conditional inactivation of IL-4 and IL-13 signaling does not impair the response of $C D 11 b^{\text {hi }}$ DC2s to $N b$ immunization, but prevents the contribution of the highly stimulatory $\mathrm{CD} 11 b^{\text {lo }}$ subset.

To better understand the increased responses to $\mathrm{Ca}$ in $/ / 4 \mathrm{ra}^{\mathrm{f} / \text { - }} \mathrm{zDC}$-Cre mice, we compared expression of relevant transcripts in the $C D 11 b^{\text {hi }}$ and $C D 11 b^{\text {lo }} D C 2$ subsets. As shown in Figure 5K, expression of the Toll-like receptor (TLR) transcripts T/r1, TIr2, TIr6 and TIr11 and the TLR chaperone Unc93b1 was higher in CD11b hi compared to CD11b ${ }^{\text {lo }}$ DC2s. As these 
TLRs are involved in the recognition of bacterial and fungal components and the production of pro-inflammatory cytokines including IL-6 and IL-12p40 upon recognition of fungal ligands (Pietrella et al., 2006), a higher representation of the CD11 bi ${ }^{\text {hi }}$ subset, as in thel/4ra ${ }^{\mathrm{f} /-}$ zDCCre mice, could favour IL-17A responses in these mice.

\section{The transcriptome of human skin DC2s is enriched in IL-4/IL-13 signature genes.}

Although classical DC2s have been identified in humans (Dutertre et al., 2019), a human equivalent of the CD11b ${ }^{\text {low }}$ subset has not been described. To assess whether skin-specific IL-4 and IL-13 signalling pathways could be observed also in human DC2s, we identifed four published transcriptomic datasets that include tissue DC2s from the skin or lung of healthy individuals and compared their transcriptomic signature to the signature of control DC2s from blood or spleen within the same study (GSE35457, GSE85305 and (Chen et al., 2020) skin; GSE43184 lung).

Gene Set Enrichment Analysis (GSEA) (Subramanian et al., 2005) and the Reactome database (Jassal et al., 2020) allow for the unbiased identification of manually curated pathways that are preferentially enriched in datasets of interest. By comparing human tissue DC2s to their respective blood or spleen controls, we found that the majority of enriched pathways were unique to each individual comparison, reflecting inter-study variations (Figure 6A). Only one pathway, the IL-10 signaling pathway, was enriched in all four comparisons. In addition, four pathways were significantly enriched only in the three comparisons of human skin DC2s (or total DCs) to blood or spleen DC2s (or DCs); these included the 'IL-4 and IL-13 signalling', 'Signaling by PDGF', 'TRIF TICAM1 mediated TLR4 signaling' and 'Regulation of insulin like growth factor' pathways. GSEA enrichment plots for 'IL-4 and IL-13 signaling' genes further indicated that skin, but not lung, DC2s showed a significant enrichment for this pathway (Figure 6B). Heatmaps showing expression of the GSEA core-enrichment genes from the 'IL-4 and IL-13 signalling' pathway across the three microarray and one scRNA-seq studies revealed that similar transcripts were enriched in skin DC2 datasets compared to blood, spleen or lung DC2s (Figure 6C and Figure S6A). Interestingly, IRF4 was enriched in human skin DC2s compared to blood, spleen or lung $D C 2 s$, and was also enriched in murine skin-dLN CD11b low $D C 2 s$ compared to other DC2s (Figure 3D), suggesting further similarities between human and murine DC2s in the skin.

Previous studies have detected expression of IL13 transcripts in human skin from healthy indivdiuals (He et al., 2020; Rojahn et al., 2020), although it was unclear whether it was associated with T cells or ILCs due to the transcriptional similarity of these populations. We interrogated the dataset from one of these studies, which includes scRNA-seq data from skin biopsies and suction blisters of healthy individuals (Rojahn et al., 2020), for expression of IL-13 signalling genes in the immune and non-immune skin cell compartments. Similar to 
Rojahn et al, we identified several clusters of myeloid cells including monocytes, CDC1s, CDC2s, LCs and a small cluster of CCR $7^{\text {high }} /$ mature DCs (Figure 6D and S6B-C). Several genes that we identified as important for the IL-13-dependent development of DC2s in murine skin were also expressed in this myeloid cell compartment including III3RA1, which was more highly expressed on myeloid cells than any other skin cell population, IL4R, STAT6 and KLF4 (Figure 6E and S6C). In the lymphocyte compartment, we identified four clusters corresponding to T-cells (CD3+ FOXP3-), T-regs (CD3+ FOXP3+), NK cells (CD3- KLRD1+) and ILCS (CD3-KLRD1- IL7R ${ }^{\text {high }}$ and KLRB1+) (Figure 6F). Cells within the ILC cluster also expressed $K I T$, IL2RA, GATA3, which have been associated with KIT+ ILC3s and IL2RA+ GATA3+ ILC2S (Teunissen et al., 2014). Unlike flow cytometry (Wojno et al., 2015), PTGDR2 encoding the prostaglandin D2 receptor CRTH2 was not sufficient to identify ILC2s by scRNA-seq due to its low expression. IL13 expression was detected at low level in $5.12 \%$ and $2.96 \%$ of the T cell and ILC clusters in healthy skin, respectively. By contrast, only T cells, but not ILCs, expressed IL4 (Figure 6F-G).

Taken together, our reanalysis of five published human datasets shows that human DC2s from healthy adult skin express an IL-4/IL-13 gene signature compared to DC2s from other tissues, suggesting that the transcriptome of human skin DC2s could be influenced by local IL-13 as demonstrated in mice. 


\section{DISCUSSION}

In this paper we report that the steady-state production of IL-13 in murine dermis is necessary and sufficient for the development of a population of dermal DC2s that is unique to skin and is characterized by low expression of CD11b. We show that skin-migratory CD11b ${ }^{\text {low }}$ DC2s express a STAT6-dependent signature and that their development is impaired in mice that are either STAT6 deficient, IL-13 deficient or do not express a functional IL-13 receptor. The inability of DC2s to sense IL-13 in STAT6-KO or IL-4R $\alpha$ conditional $\mathrm{KO}$ mice is functionally consequential and results in impaired IL-4+ and increased IL-17A+ Th cell responses in skin-dLN. By re-analysing published transcriptomic data, we also show that dermal DC2s in healthy human skin express an IL-13-dependent transcriptional signature and skin ILCs express IL-13, thus suggesting that DCs in healthy murine and human skin are exposed to similar IL-13 environments in the steady-state, and might exhibit similar functional properties.

The heterogeneity of the IRF4-dependent DC2 subset across different tissues is well recognised (Alcantara-Hernandez et al., 2017; Guilliams et al., 2016; Heidkamp et al., 2016) and presumably develops in response to tissue-specific signals and growth factors that remain often uncharacterised (Sichien et al., 2017). A notable example of DC2 specialization is the $\mathrm{CD}_{103}{ }^{+} \mathrm{CD} 11 \mathrm{~b}^{+} \mathrm{DC} 2$ subset unique to the SI lamina propria and mesenteric LN (Bain et al., 2017). In the absence of TGF $\beta R$ signalling, SI DC2s remain CD11 $b^{+} C D 103^{-}$and are unable to promote the development of Fox $3^{+} \mathrm{T}$ regulatory cells and mucosal Th17 populations. As cytokines of the TGF $\beta$ family are ubiquitously expressed in epithelia and are essential for the development and function of multiple tissues, they are well suited to a role in the tissue adaptation of DC populations. Unlike TGF $\beta$ s, IL-13 does not have a recognized role in tissue homeostasis. The main known function of IL-13 is its role in allergic inflammation and parasite infection (Wynn, 2003). High IL-13 production in skin, as observed in atopic disease, is associated with fibrosis, decreased expression of filaggrin and filaggrin-like molecules by keratinocytes and loss of skin barrier function (McAleer and Irvine, 2013). However, low levels of IL-13 produced by DETCs at steady state were reported to enhance epidermal integrity (Dalessandri et al., 2016), and I/13 transcripts together with low but measurable protein levels are constitutively expressed by dermal ILC2s in healthy murine skin (Roediger et al., 2013) and freshly explanted human ILC2s (Teunissen et al., 2014), raising the question of a potential homeostatic function for IL-13 (Strid et al., 2016). Here we provide evidence that homeostatic IL-13 production in skin has a clear immunological effect, firstly by supporting dermal DC2 differentiation at steady state, and secondly by regulating the ability of DC2s to induce immune responses after immunization. To our knowledge, this is the first report of a lymphocyte-derived cytokine contributing to tissue-specific DC2 development in the steady state, and the first evidence of skin as an "IL-13-conditioned" tissue in nonpathological conditions. It remains to be established how the function of other DC and nonDC poulations in skin might also be affected by IL-13 signaling. 
The DC2 phenotype in STAT6-KO and IL-4R $\alpha$ conditional KO mice was similar to mice that are total or conditional KO for KLF4, a transcription factor required for the development of $C D 11 b^{\text {low }} C D 24^{\text {low }}$ 'Double Negative' dermal DC2s and for the induction of Th2 responses (Tussiwand et al., 2015). We found that the similarity between STAT6-KO and KLF4-KO DC2s was not due to STAT6 deficiency affecting expression of KIf4 transcripts in DC2s, but to the ability of KLF4 to control the response of DC2s to IL-13 signalling in vitro and in vivo, thus revealing a previously unrecognized link between KLF4 and IL-13 signalling in DCs. The basis of the defective responsiveness to IL-13 remains unclear as expression of IL-4R $\alpha$ and IL13R $\alpha 1$ and phosphorylation of STAT6 were all similar in KLF4-KO and C57 DCs, but might be explained by a transcriptional cooperation between KLF4 and STAT6 as documented in M2 macrophages (Liao et al., 2011). Futher experiments are required to determine whether a cooperation between KLF4 and STAT6 controls DC2 transcriptional responses to IL-13. Additional effects of DC-specific KLF4 deletion, such as the increased DC1/DC2 ratio and decreased DC2 numbers in multiple tissues (Tussiwand et al., 2015), were not recapitulated in STAT6-KO and IL-4R $\alpha$ conditional KO mice thus may be driven by broader functions of KLF4, or its early expression during DC development and prior to preDCs exiting the BM.

Apart from a moderate difference between males and females, which was presumably due to the suppressive activity of male hormones on ILC2 function (Laffont et al., 2017), the ratio of dermal CD11 b ${ }^{\text {low }}$ to $C D 11 b^{\text {hi }}$ DC2s appeared fairly constant in different laboratories and in different mice regardless of anatomical location, implying a regulated mechanism that controls the equilibrium between the CD11b low and CD11b $b^{\text {hi }} D C 2$ subsets. Treatment with recombinant IL-13 was sufficient to rescue the development of CD11b bow DC2s in IL-13-KO mice but did not induce downregulation of CD11b on all dermal DC2s, suggesting that additional factors may influence the differentiation of CD11 b ${ }^{\text {low }} \mathrm{DC} 2 \mathrm{~s}$ such as the positioning within an appropriate niche (Dahlgren et al., 2019) or a pre-existing heterogeneity within the DC2 population (Sichien et al., 2017). The notion that IL-13 is not the only signal controlling the development of dermal CD11b $b^{\text {low }} \mathrm{DC} 2$ is also compatible with the presence of a small population of CD11b bow DC2s in STAT6-KO and IL-4R $\alpha-K O$ mice and the high number of differentially expressed genes in CD11 $b^{\text {hi }}$ DC2s from STAT6-KO compared to C57 mice, with the latter suggesting that the STAT6-KO CD11 bi population might include incompletely differentiated CD11b ${ }^{\text {low }} \mathrm{DC} 2 \mathrm{~s}$.

Our data suggest that steady-state IL-13 production is exclusive to the skin. Skin-specific expression of IL-13 by ILC2s has been reported in mice and humans (Roediger et al., 2013; Salimi et al., 2013), is consistent with the unique transcriptional profile of skin ILC2s (Ricardo-Gonzalez et al., 2018), and appears to be independent of skin microbiota or signaling from innate cytokines including TSLP, IL-33 and IL-25 ((Ricardo-Gonzalez et al., 2018) and our unpublished observations) suggesting a key role of the specific tissue environment in determining ILC function. The mechanism through which ILC-derived IL-13 
conditions skin DC2s at steady state also appears to differ from the mechanisms in lung, where allergen and IL-33-dependent IL-13 production by ILC2s were necessary for DC migration to the dLN (Halim et al., 2014) and for the later recruitment and activation of Th2 cells in non-lymphoid tissue (Halim et al., 2016; Halim et al., 2018; Nieuwenhuizen et al., 2018; Oliphant et al., 2014). While a STAT6 signature was detectable in CD11b low DC2s in naïve mice, our previous transcriptomic analyses of DC2 subsets in PBS and $\mathrm{Nb}$-immunized mice failed to detect an IL-4/IL-13-dependent signature in Nb-treated vs PBS mice (Connor et al., 2017), suggesting that DC2s were either not exposed to, or not further affected by, these cytokines after immunization. Together, these observations suggest that, compared to lung, the skin environment is intrinsically predisposed to Th2 induction, although both skin and lung can sustain Th2 priming in the appropriate conditions. Consistent with this view, IL13 signaling enabled the increased expression of costimulatory molecules on CD11blow $D C 2 s$, both at steady state and after $\mathrm{Nb}$-AF488 uptake. The increased responsiveness of CD $11 b^{\text {low }}$ DC2s to TSLP (Ochiai et al., 2014), an epithelial cytokine with a known role in promoting Th2 responses (Ziegler, 2012) may underpin this observation. In addition, the higher expression of several T/r transcripts in CD11 $b^{\text {hi }}$ compared to CD11 blow DC2s suggests a lower responsiveness of $C D 11 b^{\text {low }} \mathrm{DC} 2 \mathrm{~s}$ to microbial stimuli, which was observed in our previous study (Hilligan et al., 2020) and is a likely consequence of IL-13 signaling (Leyva-Castillo et al., 2020), providing a plausible mechanism for the increased IL-17A and ROR $\gamma \mathrm{t}+$ responses in IL-4R $\alpha$ conditional KO mice.

The observation of a skewed ability of skin DC2 to induce immune responses raises the question of how the low-dose, steady-state IL-13 that imparts a pro-Th2 environment in naïve skin could be advantageous for skin homeostasis given that it appears to be conserved across mice and humans. The skin environment plays an important role in the response to commensals such as S. epidermidis (Harrison et al., 2019; Naik et al., 2015) in which specific T cells produce IL-17A under homeostatic conditions for antimicrobial defense and also express Th2 potential for tissue repair. Within the context of a response to commensals, IL13-dependent DC conditioning might promote equilibrium by preventing excessive IL-17A production and consequent neutrophil infiltration, thereby avoiding damaging responses to commensals while supporting expression of repair-promoting cytokines and growth factors upon tissue injury (Harrison et al., 2019).

In addition to the potentially beneficial regulation of Th17 responses in skin, our data also reveal a mechanism for the preferential priming of Th2 responses after skin exposure. In skin, Th2 responses are reported to curb entry of helminth parasites (Obata-Ninomiya et al., 2013), or promote itch and scratch responses to insect bites or ticks to favour their removal and repair the resulting damage (Allen and Wynn, 2011; Karasuyama et al., 2020). However, Th2 responses can also cause pathology and develop into damaging allergic responses. While a preferred route for allergic sensitization in humans has not been established, epidemiological studies suggest that delayed introduction of some foods to infants may 
increase the likelihood of allergy development by providing greater opportunity for contact through skin (Du Toit et al., 2008). Similarly, genetic variations that decrease the barrier function of skin are associated with increased incidence of allergic disease that is not limited to skin but extends to respiratory and food allergy (Irvine et al., 2011; McAleer and Irvine, 2013). Our study provides a potential mechanism for these observations by documenting an IL-13-dependent and tissue-specific conditioning of dermal DC2s that leads to Th2-biased functional properties.

In conclusion, steady-state IL-13 production by dermal ILC2s underpins the development of a CD11 $b^{\text {low }} \mathrm{DC} 2$ subset that is unique to skin, and preferentially directs mouse $\mathrm{CD} 4^{+} \mathrm{T}$ cell differentiation to an IL-4+ Th2 phenotype while inhibiting Th17 responses. The functional consequences of this circuit in humans, in which an IL-13-dependent signature in skin DC2 is also apparent, are yet to be characterised. However, genome-wide association studies showing an opposing impact of IL13 gene polymorphisms in Atopic dermatitis vs Psoriasis (Baurecht et al., 2015), two skin diseases respectively dominated by dysregulated activation of Th2 and Th17 cells, respectively, suggest a similar regulation in humans and an important role of this mechanisms in the propensity to disease. 


\section{ACKNOWLEDGEMENTS}

The authors wish to thank Prof Graham Ogg, Oxford University, for sharing raw data of the scRNA-seq analysis of human skin blister (Chen et al, 2020); Dr. Patrick M. Brunner and Dr. Vera Vorstandlechner, Department of Dermatology, Medical University of Vienna, for their advice on single cell QC filtering and data analysis of scRNA-seq data of human skin biopsies and blister cells (GSE153760, Rojahn et al, 2020); Yueqi Wang, Google, for developing the Shiny browser for single cell RNAseq data, and providing debugging help, and all colleagues at the Malaghan Institute of Medical Research for discussion and suggestions. We also thank Dr. Olivier Gasser and the NIH Tetramer Facility for providing the 5-OP-RU-loaded tetramers for MAIT cell identification. The MR1 tetramer technology was developed jointly by Dr. James McCluskey, Dr. Jamie Rossjohn and Dr. David Fairlie, and the material was produced by the NIH Tetramer Core Facility as permitted to be distributed by the University of Melbourne, Australia. Finaly, we gratefully acknowledge the flow cytometry support of the members of the Hugh Green Cytometry Centre and the expert animal husbandry of the Biomedical Research Unit.

This work was funded by an Independent Research Organization grant from the Health Research Council of New Zealand (HRC) to the Malaghan Institute, a Health Research Council of NZ project grant to FR, a Malaghan Institute Postdoctoral Fellowship to KLH, the Marjorie Barclay Trust, and the Intramural Research Program of the NIAID, NIH, USA.

\section{AUTHOR CONTRIBUTIONS}

Conceptualization: JUM, OL, FR; software: SIO, DAE; formal analysis: JUM, SIO, DAE, LM-E; Investigation: JUM, OL, KLH, JSC, RGD, JY, GRW, LME, KAW, EJH, SCT, SCC, LMC; Resources: CRM, FB, AS, RT, DJ, MRH, GLG; data curation: SIO, DAE; writing the original draft: JUM, FR; reviewing \& editing the original draft: JUM, OL, FR with input from all authors; visualization JUM, OL, KLH, SIO, DAE; supervision: MRH, FR; funding acquisition: AS, FR.

\section{DECLARATION OF INTEREST}

The authors declare no competing interests. 


\section{MATERIALS AND METHODS}

\section{Mice}

Mice were bred and housed under specific pathogen-free conditions at the Malaghan Institute of Medical Research and provided with water and feed ad libitum. All mice were between 6 and 14 weeks of age and were age- and sex-matched within experiments. C57BL/6J, BALB/cByJ, B6-SJ ptprca, CD11C-Cre (B6.Cg-Tg(Itgax-cre)1-1Reiz/J), IRF4-flox (B6.129S1-Irf4 $\left.{ }^{\mathrm{tm} 1 \mathrm{Rdf}} / \mathrm{J}\right)$, STAT6-KO (B6.129S2(C)-Stat6 $\left.{ }^{\mathrm{tm} 1 \mathrm{Gru}} / \mathrm{J}\right)$ and zDC-Cre (B6.Cg-

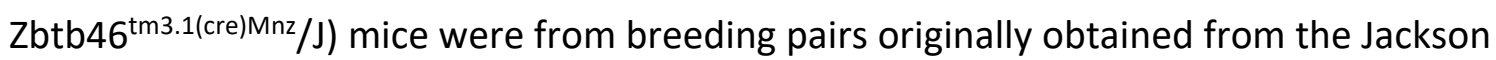
Laboratories, USA. 4C13R reporters ( $\operatorname{Tg}(I 14-A m C y a n, I I 13-D s R e d *) 1 W e p$ (Roediger et al., 2013) and IL-4-KO (II ${ }^{\text {tm1.1Wep }}$ (Hu-Li et al., 2001) mice were from breeding pairs kindly provided by Dr William Paul, NIAID, NIH, Bethesda USA; IL4Ra-KO (II4ra ${ }^{\text {tm1Fbb) }}$ (Mohrs et al., 1999) and IL-4R $\alpha$-flox (II4ra ${ }^{\text {tm2Fbb }}$ ) (Herbert et al., 2004) were from breeding pairs kindly provided by Dr Frank Brombacher, University of Capetown, ZA; MHCII-KO (H2-Aatm1Blt) (Kontgen et al., 1993) were bred from pairs originally provided by Dr Horst Bluethmann, Hoffman-La Roche Ltd, Basel, Switzerland. Itgax-cre ${ }^{+/-}$Irf $4^{\mathrm{fl} / \mathrm{fl} \text { or } \mathrm{fl} /-}\left(\mathrm{IRF} 4^{\Delta \mathrm{CD} 11 \mathrm{c}}\right)$ and $/ \mathrm{rf} 4^{\mathrm{fl} / \mathrm{fl} \text { or } \mathrm{fl} /-}$ $\left(I R F 4{ }^{\mathrm{WT}}\right)$ mice were generated by crossing Itgax-cre to Irf $4^{\mathrm{fl}}$ for two generations. $z B T B 46-$ $\mathrm{cre}^{+/-} \| 4 \mathrm{r}^{\mathrm{fl} /-}\left(\mathrm{IRF} 4^{\Delta \mathrm{ZDC}}\right)$ and $/ / 4 \mathrm{r}^{\mathrm{fl} /-}$ mice were generated by crossing $\mathrm{Zbtb}$-cre females to $/ r f 4^{\mathrm{fl}}$ for two generations.

For some experiments, $\mathrm{C} 57 \mathrm{BL} / 6 \mathrm{Tac}$ and $\mathrm{BALB} / \mathrm{c}$ were obtained from Taconic Biosciences (Rensselaer, NY) while B6.SJL-Ptprc ${ }^{a}$ Pepc $c^{b}$ BoyJ, RAG1-KO on C57BL6/J background, and IL13Ra1-KO on BALB/c background were from the NIAID contract facility at Taconic Biosciences.

Experimental protocols were approved by the Victoria University of Wellington Animal Ethics Committee or the NIAID Animal Care and Use Committee and performed according to institutional guidelines.

\section{Generation of IL-13 KO mice.}

IL-13-KO mice were generated by the Australian Phenomics Network and Monash Genome Modification Platform, Melbourne, Australia, using the strategy illustrated in Figure S3B. CAS9 enzyme, guide RNA (gRNA) 1 targeting intron 1 (5' - AGAGUCUUGGAGCUGAAAGA -3') and gRNA2 targeting intron 3 (5' - CUUAGAGCGUUACAAGUCC- $3^{\prime}$ ) of the murine $/ / 13$ gene were injected into $\mathrm{C} 57 \mathrm{BL} / 6$ fertilized eggs to remove exon 2 and 3 . Mice were screened for exon deletion by PCR using the primers P1 (5'-GAGGCTGGCATGGTGGTTTC-3') and P2 (5'TGGAGACCTGTGAAACGGCA-3'). Of two founder mice carrying exon 2-3 deletions, only one gave viable progeny carrying the mutation when crossed to $\mathrm{C} 57 \mathrm{BL} / 6$. These heterozygous mice were mated to each other to generate IL-13-KO mice carrying a homozygous exon 2-3 deletion.

\section{BM Chimeric Mice}


BM cell suspensions were prepared by flushing cut femurs and tibias with unsupplemented IMDM (Gibco) and filtering through a sterile $70 \mu \mathrm{m}$ cell strainer (Falcon). Recipient mice aged 10-12 weeks were treated with two doses of 5.5 Grey using a Gammacell ${ }^{\circledR} 3000$ Elan irradiator (MDS Nordion) given 3 hours apart. One day after irradiation, mice received 1-8 $\mathrm{x}$ $10^{6} \mathrm{KIf} 4^{f l}$ Vav-iCre or $\mathrm{KIf} 4^{\mathrm{fl}} \mathrm{BM}$ cells (Tussiwand et al., 2015) from gender-matched donor mice as detailed in the text and Figure legends.

For the generation of allogeneic mixed-BM chimeras, BM donor mice were depleted of $\mathrm{T}$ cells by intraperitoneal injection of $200 \mu \mathrm{g}$ InVivoMAb anti-mouse Thy-1 (Clone T24/31, BioXCell) on day -5 and -2 before BM collection. Recipient mice were injected with $100 \mu \mathrm{g}$ anti-mouse Thy-1 i.p. one day after BM transfer. Chimeric mice were housed in individually ventilated cages and supplied with $2 \mathrm{mg} / \mathrm{mL}$ Neomycin trisulfate-supplemented drinking water for the first 3 weeks. For steady-state DC profiling and BMDC generation, chimeric mice were used at least 8 weeks after reconstitution, or immunized after 12 weeks of reconstitution for the characterization of $\mathrm{CD} 4^{+} \mathrm{T}$ cell responses.

\section{Immunizations and in vivo treatments}

Mice were anesthetized with $100 \mu \mathrm{g} / \mathrm{g}$ body weight Ketamine and $3 \mu \mathrm{g} / \mathrm{g}$ body weight Xylazine (both Provet) by intraperitoneal injection and injected with $4 \times 10^{6} \mathrm{CFU}$ heat-killed Ms, $1 \times 10^{7}$ heat-killed Ca or 300 non-viable Nb L3 larvae in $30 \mu \mathrm{l}$ PBS intradermally in the ear pinna as previously described (Hilligan et al., 2020). PBS was injected into the ear pinna of control animals. $60 \mu \mathrm{l}$ of PBS or $20 \mu \mathrm{g} \mathrm{IL-13}$ fusion protein (Absolute Antibody) in the same volume were injected intraperitoneally on day $0,60 \mu$ of PBS or $10 \mu \mathrm{g} \mathrm{IL-13}$ fusion protein were injected on day 2 and 3 and tissues harvested on day 4 .

\section{Preparation of immunogens}

M. smegmatis (Ms, mc2155) was grown in LB broth (Difco LB Lennox - low salt, BD) under agitation at $37^{\circ} \mathrm{C}$ overnight. Bacteria were washed three times in PBS containing $0.05 \%$ Tween 80 and heat-killed at $75^{\circ} \mathrm{C}$ for 1 hour and stored at $-70^{\circ} \mathrm{C}$. N. brasiliensis $(\mathrm{Nb})$ infective L3 larvae were prepared as described (Camberis et al., 2013), washed three time with sterile PBS followed by three washes with antibiotic wash buffer (AWB: PBS supplemented with $100 \mathrm{\mu g} / \mathrm{ml}$ Gentamcycin (Sigma-Aldrich) and $500 \mathrm{U} / \mathrm{ml}$ Penicillin-Streptomycin (Gibco)). Larvae were incubated in AWB twice for 1 hour at room temperature, washed four times with sterile PBS and inactivated by three freeze-thaw cycles. $C$. albicans $(\mathrm{Ca})$ was propagated by inoculating sterile yeast extract-peptone-dextrose broth (Difco YPD, BD) and incubating under agitation at $30^{\circ} \mathrm{C}$ for 72 hours. Yeasts were washed in PBS and heat-killed at $75^{\circ} \mathrm{C}$ for 1 hour. For fluorescent labelling of antigens, non-viable $\mathrm{Nb}$ were incubated in $0.05 \mathrm{M} \mathrm{NaHCO}_{3}$ buffer and $0.1 \mathrm{mg} \mathrm{AF488} \mathrm{NHS} \mathrm{Ester} \mathrm{(Molecular} \mathrm{Probes,} \mathrm{Invitrogen)} \mathrm{for} 15$ minutes and washed with $0.1 \mathrm{M}$ Tris buffer according to the manufacturer's instructions.

\section{Tissue sampling and processing}


To prepare single cell suspensions for DC assessment, auricular or inguinal LNs draining the skin, mediastinal LNs draining the lung or mesenteric LNs specifically draining the small intestine were disrupted with $18 \mathrm{G}$ needles and digested in IMDM containing $100 \mu \mathrm{g} / \mathrm{mL}$ Liberase TL and $100 \mu \mathrm{g} / \mathrm{mL}$ DNase I (both Sigma-Aldrich) for 30 minutes at $37^{\circ} \mathrm{C}$. Cells were collected, filtered through a $70 \mu \mathrm{m}$ cell strainer, washed with FACS buffer (PBS supplemented with 2\% FCS, 2 mM EDTA and 0.01\% Sodium Azide (all Gibco)) and maintained at $4^{\circ} \mathrm{C}$. For skin preparations, ears were collected and split into ventral and dorsal halves, cut into small pieces and digested in $2 \mathrm{ml} \mathrm{HBSS} \mathrm{(Gibco)} \mathrm{containing} 2 \mathrm{mg} / \mathrm{mL}$ Collagenase IV (Sigma-Aldrich) and $100 \mu \mathrm{g} / \mathrm{mL}$ DNase I (Sigma-Aldrich) for 30 minutes at $37^{\circ} \mathrm{C}$ and $250 \mathrm{rpm}$ in a shaking incubator. The suspension was collected, dissociated using a syringe and $18 \mathrm{G}$ needle, filtered through a $70 \mu \mathrm{m}$ cell strainer, washed with FACS buffer and maintained at $4^{\circ} \mathrm{C}$. Lungs were collected after perfusion, cut into small pieces and digested in $1 \mathrm{ml}$ IMDM (Gibco) containing $500 \mu \mathrm{g} / \mathrm{mL}$ Liberase TL and $500 \mu \mathrm{g} / \mathrm{mL}$ DNase I (both Sigma-Aldrich) for 45 minutes at $37^{\circ} \mathrm{C}$ and $150 \mathrm{rpm}$ in a shaking incubator. Cells were collected, filtered through a $70 \mu \mathrm{m}$ cell strainer, washed with FACS buffer and maintained at $4^{\circ} \mathrm{C}$. Small intestine preparations were performed as previously described (Ferrer-Font et al., 2020). Small intestinal segments were excised, Peyer's patches were removed and the intestines were opened longitudinally. Segments were when washed in PBS, cut into $0.5 \mathrm{~cm}$ pieces, collected in cold HBSS and washed twice with HBSS containing 2 mM EDTA (both Gibco) for 15 minutes at $37^{\circ} \mathrm{C}$ and $200 \mathrm{rpm}$ in a shaking incubator to dissociate the epithelium. Segments were then digested in RPMI (Gibco) containing 10\% FCS (Gibco), 1 $\mathrm{mg} / \mathrm{ml}$ Collagenase VIII and $50 \mu \mathrm{g} / \mathrm{ml}$ DNase I (both Sigma-Aldrich) for $15-20$ minutes at $37^{\circ} \mathrm{C}$ and $200 \mathrm{rpm}$ in a shaking incubator. The suspension was then filtered through a $100 \mu \mathrm{m}$ and $40 \mu \mathrm{m}$ cell strainer, washed with FACS buffer and maintained at $4^{\circ} \mathrm{C}$.

Skin-dLN preparations for the assessment of T cell responses were generated by pressing the LNs through a $70 \mu \mathrm{m}$ cell strainer to obtain single cell suspensions, washed with FACS buffer and maintained at $4^{\circ} \mathrm{C}$. For the assessment of intracellular cytokines in T cells, LN single cell suspensions were incubated in TCM containing $50 \mathrm{ng} / \mathrm{mL}$ PMA (Sigma-Aldrich), 1 $\mu \mathrm{g} / \mathrm{mL}$ ionomycin (Sigma-Aldrich) and $1 \mu \mathrm{L} / \mathrm{mL}$ GolgiStop (BD Pharmingen) for 5 hours before proceeding with surface and intracellular flow cytometry staining.

\section{FLT3L bone marrow cultures}

BM cells were suspended in Red Blood Cell Lysing Buffer Hybri-Max ${ }^{\mathrm{TM}}$ (Sigma-Aldrich) for 1 min at RT, washed and resuspended in tissue culture medium (TCM: IMDM supplemented with $5 \%$ fetal calf serum (FCS), 1\% Penicillin-Streptomycin and $55 \mu \mathrm{M}$ 2-Mercaptoethanol (all Gibco) supplemented with 4\% FLT3L supernatant (generated from the cell line CHO flag Flk2.clone 5 kindly provided by Prof Nic Nicola, WEHI). BM cells were cultured in 6-well plates (Corning) at $5 \times 10^{6} /$ well in $5 \mathrm{ml} \mathrm{TCM}$, and incubated at $37^{\circ} \mathrm{C}$ in $95 \%$ humidity and $5 \%$ $\mathrm{CO}_{2}$ for 9 days. Cultures were fed every three days by removing $2 \mathrm{~mL}$ of medium and adding $2 \mathrm{~mL}$ of TCM containing 10\% FLT3L supernatant. Recombinant murine IL-13 (Peprotech) was added at different times and concentrations as indicated in each experiment. 


\section{Phospho-STAT6 Immunofluorescence}

At day 9, unstimulated FLT3L bone marrow DCs (BMDCs) were harvested and one million cells were resuspended in $1 \mathrm{~mL}$ of TCM in 24 wells plates and stimulated with recombinant mouse IL-13 (Peprotech) at a concentration of $100 \mathrm{ng} / \mathrm{mL}$ for $30 \mathrm{~min}$ at $37^{\circ} \mathrm{C} .1 \times 10^{5}$ cells were then cytocentrifuged onto microscope glass slides (Trajan), encircled with a PAP pen (Daido Sangyo), fixed with 4\% paraformaldehyde (ThermoFisher) for $10 \mathrm{~min}$ at room temperature, and permeabilized with $100 \%$ methanol at $4^{\circ} \mathrm{C}$ for $10 \mathrm{~min}$, as previously described (Schmidt et al., 2019). Blocking was performed for 1 hour at room temperature using SuperBlock T20 (ThermoFisher), before incubation with primary antibody (anti-pSTAT6 Tyr641, ThermoFisher) for 1 hour at room temperature at 1:100 dilution in PBS and washed three times with PBS. Slides were then incubated with secondary antibody anti-rabbit Alexa Fluor 647 (ThermoFisher), anti-CD172a Alexa Fluor 594 (Biolegend) and DAPI (0.5 $\mu \mathrm{g} / \mathrm{ml}$, Merck). Slides were mounted with Faramount (Agilent) and covered with a glass coverslip (22x22, Thermofisher). Images were obtained using the FV3000 confocal microscope (Olympus) with a 10x objective, using the 405, 594 and $640 \mathrm{~nm}$ laser lines and $3 \mathrm{GaAsp}$ photomultiplier detectors. Images were acquired with a 16-bit depth, a line average of 3 using sequential scanning and a pixel dimension of 2048x2048 (1 $\mu \mathrm{m}=1.6091$ pixels). Acquired images were first pre-processed with the FIJI imaging processing package (v2.0.0rc-69/1.52i, (Schindelin et al., 2012)). Cell masks were generated using CellProfiler (v4.0.3, (Jones et al., 2008)), while .fcs files using the cell masks and channel images were generated with Histocat (v1.76, (Schapiro et al., 2017). Gating and cell marker statistics were performed using Flowjo software (v10.7, BD), and these statistics were imported into GraphPad Prism 8 (v8.4, GraphPad Software, Inc.) for statistical analysis.

\section{Flow cytometry and cell sorting}

Single cell suspensions were incubated in FACS buffer containing anti-mouse CD16/32 (clone 2.4G2, hybridoma culture supernatant, 1:300 dilution) to block Fc receptors prior to labelling with a mix of fluorescently labeled monoclonal antibodies for 30 minutes at $4^{\circ} \mathrm{C}$. Anti-CD3 (145-2C11), anti-CD4 (RM4-5), anti-CD11b (M1/70), anti-CD11c (HL3), anti-CD326 (G8.8), anti-IA/IE (M5/114.15.2), anti-NK1.1 (PK136), anti-CD45R (RA3-6B2), anti-CD86 (GL1), anti-Ly6A-E (D7), anti-CD44 (IM7), anti-CD103 (M290) and anti-NK1.1 (PK136) were from BD Biosciences. Anti-CD64 (X54-5/7.1), anti-CD8 (2.43), anti-Ly6C (HK1.4), anti-Ly6C (AL-21), anti-CD11C (N418), anti-CD26 (H194-112), anti-CD172a (P84), anti-CD45.1 (A20), anti CD45.2 (104), anti-CD45 (30-F11), anti-CD278 (C398.4A), anti-CD90.2 (30-H12), antiCD90.2 (53-2.1), anti-CD124 (I015F8), anti-CD278 (7E.17G9), anti-CD24 (30-F1), anti-CD25 (3C7), anti-TCR $\delta$ (GL3), anti-CD49b (DX5), anti-Ly6C/Ly6G (RB6-8C5), anti-CD200R3 (Ba13), anti-FceRI $\alpha$ (MAR-1), anti-CD317 (927), anti-CD117 (2B8), anti-CD19 (6D5) and anti-XCR1 (ZET) were from BioLegend. Anti-KLRG1 (2F1), anti-CD19 (ID3), anti-Siglec-F (E50-2440), antiTCR $\beta$ (H57-597), anti-CD127 (A7R34), anti-CD273 (TY25), anti-Ly6G (1A8), anti-CD206 (MR6F3), anti-IL33R (RMST2-33) and anti-CD213a (13MOKA) were from ThermoFisher. 5- 
OP-RU-loaded MR1 tetramer conjugated to BV421 was kindly provided by Dr. Olivier Gasser. Dead cells were excluded from analysis using DAPI (Molecular Probes) or ZOMBIE NIR (Biolegend). For staining of intracellular cytokines, cells were stained with LIVE/DEAD ${ }^{\text {TM }}$ Fixable Blue (Molecular Probes) prior to staining of cell surface molecules. Cells were then fixed and permeabilized using a BD Cytofix/Cytoperm kit (BD Pharmingen) and stained for intracellular cytokines. Anti-IL-4 (11B11) and anti-IFN $\gamma$ (XMG1.2) were from BD Biosciences; anti-IL-13 (eBio13A) and anti-IL-17A (eBio17B7) were from ThermoFisher. For staining of transcription factors, cells were stained with LIVE/DEAD ${ }^{\text {TM }}$ Fixable Blue (ThermoFisher) prior to staining of cell surface molecules. Cells were then fixed and permeabilized using a True-Nuclear ${ }^{\mathrm{TM}}$ Transcription Factor Buffer Set (BioLegend) and stained with anti-ROR $\gamma \mathrm{t}$ (Q31-378) and anti-GATA3 (C50-823), both from BD Biosciences. Single-stain controls were prepared with UltraComp eBeads (ThermoFisher) following the manufacturer's instructions, or cells, if this resulted in a brighter signal, and were used to calculate a compensation matrix. Sample acquisition was performed on a LSR Fortessa or FACSymphony (both BD Biosciences) using BD FACS DIVA software or a 3L or $5 \mathrm{~L}$ Aurora Spectral Flow Cytometer (Cytek Biosciences) using SpectroFlo software v2.2. Final analysis and graphical output were performed using FlowJo software (v10.7, BD), OMIQ (v2020, $\mathrm{OMIQ}$, Inc.).

For sorting of DC subsets for quantitative RT-PCR analysis, cells were stained as described and DC2 subsets from skin dLN were sorted as indicated in Figure S1. Cells were directly sorted into RNA lysis buffer (Zymo Research) using a BD INFLUX Cell sorter (BD Biosciences) and frozen at $-80^{\circ} \mathrm{C}$ until RNA extraction. Three biological replicates were prepared in two separate DC sorting experiments and purity of target cell population was $>95 \%$.

For sorting of DC subsets for RNA sequencing, cells were stained as described and presorted as $\mathrm{MHCII}^{\text {hi }}$ migratory DCs from skin, lung and SI dLNs as indicated in Figure S1 using a BD INFLUX Cell sorter (BD Biosciences). Cells were resuspended in FACS buffer and the respective DC2 subets were sorted directly into QIAzol Lysis Reagent (QIAGEN) and frozen at $-80^{\circ} \mathrm{C}$ until RNA extraction. Three biological replicates were prepared in three separate DC sorting experiments and purity of target cell population was $>98 \%$.

\section{RNA isolation and sequencing}

Total RNA was prepared from frozen cell pellets using the RNeasy Micro Kit (QIAGEN). RNA was quantified using a Quantus Fluorometer (Promega) and RNA integrity was checked using a Fragment Analyser (Agilent). Library preparation and RNA sequencing were contracted out to Otago Genomics, University of Otago, NZ. Samples were spiked with External RNA Controls Consortium mix (Ambion; Life Technologies) and ribosomal RNA was depleted using RiboZero (Illumina) before library preparation. Paired-end stranded RNA sequencing was performed on an Illumina HiSeq 2500 V4 sequencing system using Illumina TruSeq kits. Between 10 and 30 million read pairs were generated per sample. 


\section{Read mapping and differential expression analysis}

Paired-end raw FASTQ files were trimmed using Trimmomatic (v0.36) (Bolger et al., 2014) and aligned using STAR (v2.7.1a) (Dobin et al., 2013) to Mus musculus mm10 M16 (GRCm38.p5) genome downloaded from GENCODE. Aligned reads were quality-checked using MultiQC (v1.7) (Ewels et al., 2016) and counted using the R (v3.4.4) package Rsubread (v1.28.1) (Liao et al., 2013). Differentially expressed genes were identified using the $R$ package DESeq2 (v1.28.1) (Love et al., 2014) and the output was filtered for log2 foldchange $>1$ in either direction and adjusted $p$-values $<0.05$. VSTpk (variance-stabilized reads per thousand base pairs) values were generated from DESeq2 normalised counts using the DESeq2 Variance Stabilizing Transformation with default parameters, then dividing the resulting value by the length of the longest gene isoform in $\mathrm{kb}$. Visualizations were made using the R packages pheatmap (v1.0.12), UpSetR (v1.4.0) (Conway et al., 2017) and Tidyverse (v1.3.0). The cluster-specific gene signatures in Figure 1 and Table S1 were created using DESeq2 and comprised of all the protein-coding genes that were differentially expressed in a certain cluster compared to each of the other clusters, with a Fold Change $>2$ in either direction and adjusted $p$-value $<0.05$. VSTpk z-scores were calculated using the $R$ function base::scale(center = TRUE) on VSTpk values in a column-wise fashion for each gene.

\section{Transcription factor binding site analysis}

For in silico prediction of transcription factor binding sites, the promoter sequences of the genes of interest (Table S1) were analysed using TRANSFAC (Matys et al., 2006). Briefly, sequences from $5000 \mathrm{bp}$ to $100 \mathrm{bp}$ upstream (from $-5000 \mathrm{bp}$ to $+100 \mathrm{bp}$ ) of transcription start site were selected and analysed against a random set of genes of the same size with the Immune cell specific group of matrices ( $p<0.01$, v2020.02).

\section{Quantitative RT-PCR}

RNA was extracted from 1000 cells using the Quick-RNA Microprep Kit (Zymo Research) following the manufacturer's guidelines. cDNA was synthetized from the total amount of extracted RNA using the High capacity RNA-to-cDNA kit (ThermoFisher). qPCR targets were preamplified using SsoAdvanced Preamp supermix (Bio-rad) following the manufacturer's guidelines. As specified by the manufacturer's guidelines, preamplified cDNA was diluted 40 times and RT-qPCR was performed using SYBR Green Master Mix (ThermoFisher) and the forward (FW) and reverse (REV) primers (IDT) FW 5'-TGCTCAGGACCAGACCAATTC-3' and REV 5'-CCTGGAAATCTCTGCAGGTGT-3' for Itgax, FW 5'-CAGATCACACATGCAACAGAGAC-3' and REV 5'-TCAATTTCAAGCCCTTGCTGTG-3' for Cc/9, FW 5'-TTCATGGACCTGCACAGGATG-3' and REV 5'-GAAGTCTGCAGGGTTGTTGTG-3' for Abcg3, FW 5'-GCGAGAAGATTCCGCTGGTA-3' and REV 5'-CCGTTGACAGTCTTCCGACA-3' for Socs3, FW 5'-GCCTGCACACCTCTACATCAT-3' and REV 5'-CTGAGCATCCGTGAGTGGTG-3' for Sned1, FW 5'-GCTGTGGGTGATGGACATGAT-3' and REV 5'-TCCCTCAGAGGATGATCCCG-3' for Khdc1a, FW 5'-GCTACGCCGTCTACTGGAAC-3' and REV 5'-AGTGAGGGCAGAAAACATCCA-3' for Efna5, FW 5'-CTCTCTGGGCGAAATCTGCT-3' and REV 5'-GAGTGCTTTCGCTATGTTGTTCA-3' for CImn and FW 5'- 
TGATGGGTGTGAACCACGAG-3' and REV 5'-GCCGTATTCATTGTCATACCAGG-3' for Gapdh using a QuantStudio 7 (Applied Biosystems). Transcript levels are expressed as the ratio of $2-\Delta C T$ (Transcript of interest)/2- $\Delta C T$ (Gapdh).

All primer pairs were designed in house using Primer3Plus, blasted against mouse transcriptome with Primer-BLAST and pre-tested to assess that amplicons of the corrects size were generated and produced single peaks upon melt curve analysis.

\section{Human DC2 transcriptomic data integration}

Microarray data were downloaded from GEO: human skin and blood DC2 (healthy volunteers) (Haniffa et al., 2012) were downloaded from GEO: GSE35457, microarray data from adult human skin and spleen DC2 (healthy volunteers) (McGovern et al., 2017) were downloaded from GSE85305, and microarray data from human lung and blood DC2 (healthy volunteers) (Yu et al., 2013) were downloaded from GSE43184. Microarray data were annotated using the 'illuminaHumanv4.db' R package (v1.26.0). Single cell RNA-seq data of human skin blister and blood cDC/Monocytes from healthy volunteers (Chen et al., 2020) were obtained from the authors and normalised using the SCTransform function of Seurat v3.2.0 (Stuart et al., 2019). In order to compare with the microarray data, gene expression for each group was summarised as the mean SCT expression across all cells within the group. Human skin blister cells and biopsy from healthy volunteers (Rojahn et al., 2020) were downloaded from GEO (GSE153760) and processed using Seurat v3.2.0 (Stuart et al., 2019), following the v3.2 "Integration and Label Transfer" vignette. Cells were pre-filtered to include only cells with at least 500 features and less than 10000 counts, as well as restricting mitochondrial fraction to between 0.5 and 15 percent. Filtered cells were then transformed within-sample via SCTransform, followed by integration using the 3,000 most variable features. UMAP dimensionality reduction was carried out, using 50 principle components and running for 400 epochs. One cluster exhibiting high variance in the UMAP plot was removed, prior to visualising the UMAP, coloured by both cluster and expression for selected genes. Feature and dot plots were created using tools integrated within Seurat.

\section{Gene Set Enrichment analysis}

GSEA (Subramanian et al., 2005) and the Reactome database (Jassal et al., 2020) were used to identify enriched signalling pathways in human DC2 from published microarray and scRNA-seq studies. Results from GSEA were processed using a custom script to recover cumulative per-marker scores for all ranked genes, so that they could be replotted with identical scales.

\section{Data access}

Raw RNA-seq data as FASTQ files have been deposited in NCBI SRA under bioproject PRJNA668222. All code and associated parameters are available at https://github.com/fronchese/Mayer_et_al_2020. 


\section{Quantification and statistical analyses}

Statistical analyses were performed using Prism 8.0 GraphPad software. Number of samples, independent experiments and statistical tests used are indicated in the figure legends. Data were analysed using ordinary one-way ANOVA when comparing multiple groups, two-way ANOVA with Sidak's multiple comparisons test when comparing two variables, or a two-tailed Student's $t$-test when comparing two groups as indicated in each figure legend. Statistical significance was defined as $p<0.05$. 


\section{REFERENCES}

Alcantara-Hernandez, M., Leylek, R., Wagar, L.E., Engleman, E.G., Keler, T., Marinkovich, M.P., Davis, M.M., Nolan, G.P., and Idoyaga, J. (2017). High-Dimensional Phenotypic Mapping of Human Dendritic Cells Reveals Interindividual Variation and Tissue Specialization. Immunity 47, 1037-1050 e1036.

Allen, J.E., and Wynn, T.A. (2011). Evolution of Th2 immunity: a rapid repair response to tissue destructive pathogens. PLoS Pathog 7, e1002003.

Ansel, K.M., Djuretic, I., Tanasa, B., and Rao, A. (2006). Regulation of Th2 differentiation and II4 locus accessibility. Annu Rev Immunol 24, 607-656.

Bagci, I.S., and Ruzicka, T. (2018). IL-31: A new key player in dermatology and beyond. J Allergy Clin Immunol 141, 858-866.

Bain, C.C., Montgomery, J., Scott, C.L., Kel, J.M., Girard-Madoux, M.J.H., Martens, L., Zangerle-Murray, T.F.P., Ober-Blobaum, J., Lindenbergh-Kortleve, D., Samsom, J.N., et al. (2017). TGFbetaR signalling controls CD103(+)CD11b(+) dendritic cell development in the intestine. Nat Commun 8, 620.

Bajana, S., Turner, S., Paul, J., Ainsua-Enrich, E., and Kovats, S. (2016). IRF4 and IRF8 Act in CD11c+ Cells To Regulate Terminal Differentiation of Lung Tissue Dendritic Cells. J Immunol 196, 1666-1677.

Baurecht, H., Hotze, M., Brand, S., Buning, C., Cormican, P., Corvin, A., Ellinghaus, D., Ellinghaus, E., Esparza-Gordillo, J., Folster-Holst, R., et al. (2015). Genome-wide comparative analysis of atopic dermatitis and psoriasis gives insight into opposing genetic mechanisms. Am J Hum Genet 96, 104-120.

Blecher-Gonen, R., Bost, P., Hilligan, K.L., David, E., Salame, T.M., Roussel, E., Connor, L.M., Mayer, J.U., Bahar Halpern, K., Toth, B., et al. (2019). Single-Cell Analysis of Diverse Pathogen Responses Defines a Molecular Roadmap for Generating Antigen-Specific Immunity. Cell Syst 8, 109-121 e106.

Bolger, A.M., Lohse, M., and Usadel, B. (2014). Trimmomatic: a flexible trimmer for Illumina sequence data. Bioinformatics 30, 2114-2120.

Chen, H., Sun, H., You, F., Sun, W., Zhou, X., Chen, L., Yang, J., Wang, Y., Tang, H., Guan, Y., et al. (2011). Activation of STAT6 by STING is critical for antiviral innate immunity. Cell 147, 436-446.

Chen, Y.L., Gomes, T., Hardman, C.S., Vieira Braga, F.A., Gutowska-Owsiak, D., Salimi, M., Gray, N., Duncan, D.A., Reynolds, G., Johnson, D., et al. (2020). Re-evaluation of human BDCA-2+ DC during acute sterile skin inflammation. J Exp Med 217.

Connor, L., Tang, S., Camberis, M., Le Gros, G., and Ronchese, F. (2014). Helminthconditioned DC prime CD4+ T cells to IL-4 production in vivo. J Immunol 193, 2709-2717.

Connor, L.M., Tang, S.C., Cognard, E., Ochiai, S., Hilligan, K.L., Old, S.I., Pellefigues, C., White, R.F., Patel, D., Smith, A.A., et al. (2017). Th2 responses are primed by skin dendritic cells with distinct transcriptional profiles. J Exp Med 214, 125-142. 
Conway, J.R., Lex, A., and Gehlenborg, N. (2017). UpSetR: an R package for the visualization of intersecting sets and their properties. Bioinformatics 33, 2938-2940.

Dahlgren, M.W., Jones, S.W., Cautivo, K.M., Dubinin, A., Ortiz-Carpena, J.F., Farhat, S., Yu, K.S., Lee, K., Wang, C., Molofsky, A.V., et al. (2019). Adventitial Stromal Cells Define Group 2 Innate Lymphoid Cell Tissue Niches. Immunity 50, 707-722 e706.

Dalessandri, T., Crawford, G., Hayes, M., Castro Seoane, R., and Strid, J. (2016). IL-13 from intraepithelial lymphocytes regulates tissue homeostasis and protects against carcinogenesis in the skin. Nat Commun 7, 12080.

Dobin, A., Davis, C.A., Schlesinger, F., Drenkow, J., Zaleski, C., Jha, S., Batut, P., Chaisson, M., and Gingeras, T.R. (2013). STAR: ultrafast universal RNA-seq aligner. Bioinformatics 29, 1521.

Du Toit, G., Katz, Y., Sasieni, P., Mesher, D., Maleki, S.J., Fisher, H.R., Fox, A.T., Turcanu, V., Amir, T., Zadik-Mnuhin, G., et al. (2008). Early consumption of peanuts in infancy is associated with a low prevalence of peanut allergy. J Allergy Clin Immunol 122, 984-991.

Dudziak, D., Kamphorst, A.O., Heidkamp, G.F., Buchholz, V.R., Trumpfheller, C., Yamazaki, S., Cheong, C., Liu, K., Lee, H.W., Park, C.G., et al. (2007). Differential antigen processing by dendritic cell subsets in vivo. Science $315,107-111$.

Dutertre, C.A., Becht, E., Irac, S.E., Khalilnezhad, A., Narang, V., Khalilnezhad, S., Ng, P.Y., van den Hoogen, L.L., Leong, J.Y., Lee, B., et al. (2019). Single-Cell Analysis of Human Mononuclear Phagocytes Reveals Subset-Defining Markers and Identifies Circulating Inflammatory Dendritic Cells. Immunity 51, 573-589 e578.

Eisenbarth, S.C. (2019). Dendritic cell subsets in T cell programming: location dictates function. Nat Rev Immunol 19, 89-103.

Ewels, P., Magnusson, M., Lundin, S., and Kaller, M. (2016). MultiQC: summarize analysis results for multiple tools and samples in a single report. Bioinformatics 32, 3047-3048.

Ferrer-Font, L., Mehta, P., Harmos, P., Schmidt, A.J., Chappell, S., Price, K.M., Hermans, I.F., Ronchese, F., le Gros, G., and Mayer, J.U. (2020). High-dimensional analysis of intestinal immune cells during helminth infection. Elife 9.

Griffith, J.W., Sokol, C.L., and Luster, A.D. (2014). Chemokines and chemokine receptors: positioning cells for host defense and immunity. Annu Rev Immunol 32, 659-702.

Guerin, A., Kerner, G., Marr, N., Markle, J.G., Fenollar, F., Wong, N., Boughorbel, S., Avery, D.T., Ma, C.S., Bougarn, S., et al. (2018). IRF4 haploinsufficiency in a family with Whipple's disease. Elife 7.

Guilliams, M., Dutertre, C.A., Scott, C.L., McGovern, N., Sichien, D., Chakarov, S., Van Gassen, S., Chen, J., Poidinger, M., De Prijck, S., et al. (2016). Unsupervised HighDimensional Analysis Aligns Dendritic Cells across Tissues and Species. Immunity 45, 669684.

Guilliams, M., Ginhoux, F., Jakubzick, C., Naik, S.H., Onai, N., Schraml, B.U., Segura, E., Tussiwand, R., and Yona, S. (2014). Dendritic cells, monocytes and macrophages: a unified nomenclature based on ontogeny. Nat Rev Immunol 14, 571-578. 
Halim, T.Y., Hwang, Y.Y., Scanlon, S.T., Zaghouani, H., Garbi, N., Fallon, P.G., and McKenzie, A.N. (2016). Group 2 innate lymphoid cells license dendritic cells to potentiate memory TH2 cell responses. Nat Immunol 17, 57-64.

Halim, T.Y., Steer, C.A., Matha, L., Gold, M.J., Martinez-Gonzalez, I., McNagny, K.M., McKenzie, A.N., and Takei, F. (2014). Group 2 innate lymphoid cells are critical for the initiation of adaptive T helper 2 cell-mediated allergic lung inflammation. Immunity 40, 425435.

Halim, T.Y.F., Rana, B.M.J., Walker, J.A., Kerscher, B., Knolle, M.D., Jolin, H.E., Serrao, E.M., Haim-Vilmovsky, L., Teichmann, S.A., Rodewald, H.R., et al. (2018). Tissue-Restricted Adaptive Type 2 Immunity Is Orchestrated by Expression of the Costimulatory Molecule OX40L on Group 2 Innate Lymphoid Cells. Immunity 48, 1195-1207 e1196.

Haniffa, M., Shin, A., Bigley, V., McGovern, N., Teo, P., See, P., Wasan, P.S., Wang, X.N., Malinarich, F., Malleret, B., et al. (2012). Human tissues contain CD141hi cross-presenting dendritic cells with functional homology to mouse CD103+ nonlymphoid dendritic cells. Immunity 37, 60-73.

Harris, N.L., Peach, R.J., and Ronchese, F. (1999). CTLA4-Ig inhibits optimal T helper 2 cell development but not protective immunity or memory response to Nippostrongylus brasiliensis. Eur J Immunol 29, 311-316.

Harrison, O.J., Linehan, J.L., Shih, H.Y., Bouladoux, N., Han, S.J., Smelkinson, M., Sen, S.K., Byrd, A.L., Enamorado, M., Yao, C., et al. (2019). Commensal-specific T cell plasticity promotes rapid tissue adaptation to injury. Science 363.

He, H., Suryawanshi, H., Morozov, P., Gay-Mimbrera, J., Del Duca, E., Kim, H.J., Kameyama, N., Estrada, Y., Der, E., Krueger, J.G., et al. (2020). Single-cell transcriptome analysis of human skin identifies novel fibroblast subpopulation and enrichment of immune subsets in atopic dermatitis. J Allergy Clin Immunol 145, 1615-1628.

Heidkamp, G.F., Sander, J., Lehmann, C.H.K., Heger, L., Eissing, N., Baranska, A., Luhr, J.J., Hoffmann, A., Reimer, K.C., Lux, A., et al. (2016). Human lymphoid organ dendritic cell identity is predominantly dictated by ontogeny, not tissue microenvironment. Sci Immunol 1.

Henri, S., Poulin, L.F., Tamoutounour, S., Ardouin, L., Guilliams, M., de Bovis, B., Devilard, E., Viret, C., Azukizawa, H., Kissenpfennig, A., et al. (2010). CD207+ CD103+ dermal dendritic cells cross-present keratinocyte-derived antigens irrespective of the presence of Langerhans cells. J Exp Med 207, 189-206.

Herbert, R.D., Holscher, C., Mohrs, M., Arendse, B., Schwegmann, A., Radwanska, M., Leeto, M., Kirsch, R., Hall, P., Mossmann, H., et al. (2004). Alternative macrophage activation is essential for survival during schistosomiasis and downmodulates Thelper 1 responses and immunopathology. Immunity 20, 623-635.

Hilligan, K., and Ronchese, F. (2020). Antigen presentation by dendritic cells and their instruction of CD4+ T helper cell responses. Cell Mol Immunol 17, 587-599.

Hilligan, K.L., Tang, S.C., Hyde, E.J., Roussel, E., Mayer, J.U., Yang, J., Wakelin, K.A., Schmidt, A.J., Connor, L.M., Sher, A., et al. (2020). Dermal IRF4+ dendritic cells and monocytes license CD4+ Thelper cells to distinct cytokine profiles. Nat Commun 11, 5637. 
Hu-Li, J., Pannetier, C., Guo, L., Lohning, M., Gu, H., Watson, C., Assenmacher, M., Radbruch, A., and Paul, W.E. (2001). Regulation of expression of IL-4 alleles: analysis using a chimeric GFP/IL-4 gene. Immunity 14, 1-11.

Irvine, A.D., McLean, W.H., and Leung, D.Y. (2011). Filaggrin mutations associated with skin and allergic diseases. N Engl J Med 365, 1315-1327.

Jassal, B., Matthews, L., Viteri, G., Gong, C., Lorente, P., Fabregat, A., Sidiropoulos, K., Cook, J., Gillespie, M., Haw, R., et al. (2020). The reactome pathway knowledgebase. Nucleic Acids Res 48, D498-D503.

Jones, T.R., Kang, I.H., Wheeler, D.B., Lindquist, R.A., Papallo, A., Sabatini, D.M., Golland, P., and Carpenter, A.E. (2008). CellProfiler Analyst: data exploration and analysis software for complex image-based screens. BMC Bioinformatics 9, 482.

Karasuyama, H., Miyake, K., and Yoshikawa, S. (2020). Immunobiology of Acquired Resistance to Ticks. Front Immunol 11, 601504.

Kontgen, F., Suss, G., Stewart, C., Steinmetz, M., and Bluethmann, H. (1993). Targeted disruption of the MHC class II Aa gene in C57BL/6 mice. Int Immunol 5, 957-964.

Laffont, S., Blanquart, E., Savignac, M., Cenac, C., Laverny, G., Metzger, D., Girard, J.P., Belz, G.T., Pelletier, L., Seillet, C., et al. (2017). Androgen signaling negatively controls group 2 innate lymphoid cells. J Exp Med 214, 1581-1592.

Lamiable, O., Mayer, J.U., Munoz-Erazo, L., and Ronchese, F. (2020). Dendritic cells in Th2 immune responses and allergic sensitization. Immunol Cell Biol 98, 807-818.

Leyva-Castillo, J.M., Das, M., Artru, E., Yoon, Y., Galand, C., and Geha, R. (2020). Mast cellderived IL-13 downregulates IL-12 production by skin dendritic cells to inhibit the Th1 response to cutaneous antigen exposure. J Allergy Clin Immunol.

Liao, X., Sharma, N., Kapadia, F., Zhou, G., Lu, Y., Hong, H., Paruchuri, K., Mahabeleshwar, G.H., Dalmas, E., Venteclef, N., et al. (2011). Kruppel-like factor 4 regulates macrophage polarization. J Clin Invest 121, 2736-2749.

Liao, Y., Smyth, G.K., and Shi, W. (2013). The Subread aligner: fast, accurate and scalable read mapping by seed-and-vote. Nucleic Acids Res 41, e108.

Love, M.I., Huber, W., and Anders, S. (2014). Moderated estimation of fold change and dispersion for RNA-seq data with DESeq2. Genome Biol 15, 550.

Matys, V., Kel-Margoulis, O.V., Fricke, E., Liebich, I., Land, S., Barre-Dirrie, A., Reuter, I., Chekmenev, D., Krull, M., Hornischer, K., et al. (2006). TRANSFAC and its module TRANSCompel: transcriptional gene regulation in eukaryotes. Nucleic Acids Res 34, D108110.

Mayer, J.U., Demiri, M., Agace, W.W., MacDonald, A.S., Svensson-Frej, M., and Milling, S.W. (2017). Different populations of $\mathrm{CD} 11 \mathrm{~b}(+)$ dendritic cells drive Th2 responses in the small intestine and colon. Nat Commun 8, 15820.

McAleer, M.A., and Irvine, A.D. (2013). The multifunctional role of filaggrin in allergic skin disease. J Allergy Clin Immunol 131, 280-291.

McCormick, S.M., and Heller, N.M. (2015). Commentary: IL-4 and IL-13 receptors and signaling. Cytokine 75, 38-50. 
McGovern, N., Shin, A., Low, G., Low, D., Duan, K., Yao, L.J., Msallam, R., Low, I., Shadan, N.B., Sumatoh, H.R., et al. (2017). Human fetal dendritic cells promote prenatal T-cell immune suppression through arginase-2. Nature 546, 662-666.

Miller, J.C., Brown, B.D., Shay, T., Gautier, E.L., Jojic, V., Cohain, A., Pandey, G., Leboeuf, M., Elpek, K.G., Helft, J., et al. (2012). Deciphering the transcriptional network of the dendritic cell lineage. Nat Immunol 13, 888-899.

Mohrs, M., Ledermann, B., Kohler, G., Dorfmuller, A., Gessner, A., and Brombacher, F. (1999). Differences between IL-4- and IL-4 receptor alpha-deficient mice in chronic leishmaniasis reveal a protective role for IL-13 receptor signaling. J Immunol 162, 73027308.

Naik, S., Bouladoux, N., Linehan, J.L., Han, S.J., Harrison, O.J., Wilhelm, C., Conlan, S., Himmelfarb, S., Byrd, A.L., Deming, C., et al. (2015). Commensal-dendritic-cell interaction specifies a unique protective skin immune signature. Nature 520, 104-108.

Naik, S.H., Sathe, P., Park, H.Y., Metcalf, D., Proietto, A.I., Dakic, A., Carotta, S., O'Keeffe, M., Bahlo, M., Papenfuss, A., et al. (2007). Development of plasmacytoid and conventional dendritic cell subtypes from single precursor cells derived in vitro and in vivo. Nat Immunol 8, 1217-1226.

Nieuwenhuizen, N.E., Kirstein, F., Hoving, J.C., and Brombacher, F. (2018). House dust mite induced allergic airway disease is attenuated in CD11c(cre)IL-4Ralpha(-/lox) mice. Sci Rep 8, 885.

Obata-Ninomiya, K., Ishiwata, K., Tsutsui, H., Nei, Y., Yoshikawa, S., Kawano, Y., Minegishi, Y., Ohta, N., Watanabe, N., Kanuka, H., et al. (2013). The skin is an important bulwark of acquired immunity against intestinal helminths. J Exp Med 210, 2583-2595.

Ochiai, S., Roediger, B., Abtin, A., Shklovskaya, E., Fazekas de St Groth, B., Yamane, H., Weninger, W., Le Gros, G., and Ronchese, F. (2014). CD326(lo)CD103(lo)CD11b(lo) dermal dendritic cells are activated by thymic stromal lymphopoietin during contact sensitization in mice. J Immunol 193, 2504-2511.

Oliphant, C.J., Hwang, Y.Y., Walker, J.A., Salimi, M., Wong, S.H., Brewer, J.M., Englezakis, A., Barlow, J.L., Hams, E., Scanlon, S.T., et al. (2014). MHCII-mediated dialog between group 2 innate lymphoid cells and CD4(+) T cells potentiates type 2 immunity and promotes parasitic helminth expulsion. Immunity 41, 283-295.

Olomski, F., Fettelschoss, V., Jonsdottir, S., Birkmann, K., Thoms, F., Marti, E., Bachmann, M.F., Kundig, T.M., and Fettelschoss-Gabriel, A. (2020). Interleukin 31 in insect bite hypersensitivity-Alleviating clinical symptoms by active vaccination against itch. Allergy 75 , 862-871.

Pellefigues, C., Tang, S.-C., Schmidt, A., White, R., Lamiable, O., Connor, L., Ruedl, C., Dobrucki, J., Le Gros, G., and Ronchese, F. (2017). TLR4, but not Neutrophil extracellular traps, promote IFN type I expression to enhance Th2 responses to Nippostrongylus brasiliensis. Frontiers in Immunology in press.

Perner, C., Flayer, C.H., Zhu, X., Aderhold, P.A., Dewan, Z.N.A., Voisin, T., Camire, R.B., Chow, O.A., Chiu, I.M., and Sokol, C.L. (2020). Substance P Release by Sensory Neurons Triggers Dendritic Cell Migration and Initiates the Type-2 Immune Response to Allergens. Immunity 53, 1063-1077 e1067. 
Persson, E.K., Uronen-Hansson, H., Semmrich, M., Rivollier, A., Hagerbrand, K., Marsal, J., Gudjonsson, S., Hakansson, U., Reizis, B., Kotarsky, K., et al. (2013). IRF4 transcriptionfactor-dependent CD103(+)CD11b(+) dendritic cells drive mucosal T helper 17 cell differentiation. Immunity 38, 958-969.

Pietrella, D., Bistoni, G., Corbucci, C., Perito, S., and Vecchiarelli, A. (2006). Candida albicans mannoprotein influences the biological function of dendritic cells. Cell Microbiol 8, 602-612.

Prussin, C., and Metcalfe, D.D. (2003). 4. IgE, mast cells, basophils, and eosinophils. J Allergy Clin Immunol 111, S486-494.

Ricardo-Gonzalez, R.R., Van Dyken, S.J., Schneider, C., Lee, J., Nussbaum, J.C., Liang, H.E., Vaka, D., Eckalbar, W.L., Molofsky, A.B., Erle, D.J., et al. (2018). Tissue signals imprint ILC2 identity with anticipatory function. Nat Immunol 19, 1093-1099.

Roediger, B., Kyle, R., Yip, K.H., Sumaria, N., Guy, T.V., Kim, B.S., Mitchell, A.J., Tay, S.S., Jain, R., Forbes-Blom, E., et al. (2013). Cutaneous immunosurveillance and regulation of inflammation by group 2 innate lymphoid cells. Nat Immunol 14, 564-573.

Rojahn, T.B., Vorstandlechner, V., Krausgruber, T., Bauer, W.M., Alkon, N., Bangert, C., Thaler, F.M., Sadeghyar, F., Fortelny, N., Gernedl, V., et al. (2020). Single-cell transcriptomics combined with interstitial fluid proteomics defines cell type-specific immune regulation in atopic dermatitis. J Allergy Clin Immunol.

Ronchese, F., Hilligan, K.L., and Mayer, J.U. (2020). Dendritic cells and the skin environment. Curr Opin Immunol 64, 56-62.

Salimi, M., Barlow, J.L., Saunders, S.P., Xue, L., Gutowska-Owsiak, D., Wang, X., Huang, L.C., Johnson, D., Scanlon, S.T., McKenzie, A.N., et al. (2013). A role for IL-25 and IL-33-driven type-2 innate lymphoid cells in atopic dermatitis. J Exp Med 210, 2939-2950.

Schapiro, D., Jackson, H.W., Raghuraman, S., Fischer, J.R., Zanotelli, V.R.T., Schulz, D., Giesen, C., Catena, R., Varga, Z., and Bodenmiller, B. (2017). histoCAT: analysis of cell phenotypes and interactions in multiplex image cytometry data. Nat Methods 14, 873-876.

Schindelin, J., Arganda-Carreras, I., Frise, E., Kaynig, V., Longair, M., Pietzsch, T., Preibisch, S., Rueden, C., Saalfeld, S., Schmid, B., et al. (2012). Fiji: an open-source platform for biologicalimage analysis. Nat Methods 9, 676-682.

Schlitzer, A., McGovern, N., Teo, P., Zelante, T., Atarashi, K., Low, D., Ho, A.W., See, P., Shin, A., Wasan, P.S., et al. (2013). IRF4 transcription factor-dependent CD11b+ dendritic cells in human and mouse control mucosal IL-17 cytokine responses. Immunity 38, 970-983.

Schlitzer, A., Sivakamasundari, V., Chen, J., Sumatoh, H.R., Schreuder, J., Lum, J., Malleret, B., Zhang, S., Larbi, A., Zolezzi, F., et al. (2015). Identification of cDC1- and cDC2-committed DC progenitors reveals early lineage priming at the common DC progenitor stage in the bone marrow. Nat Immunol 16, 718-728.

Schmidt, A.J., Mayer, J.U., Wallace, P.K., Ronchese, F., and Price, K.M. (2019). Simultaneous Polychromatic Immunofluorescent Staining of Tissue Sections and Consecutive Imaging of up to Seven Parameters by Standard Confocal Microscopy. Curr Protoc Cytom 91, e64.

Segura, E., Valladeau-Guilemond, J., Donnadieu, M.H., Sastre-Garau, X., Soumelis, V., and Amigorena, S. (2012). Characterization of resident and migratory dendritic cells in human lymph nodes. J Exp Med 209, 653-660. 
Shklovskaya, E., O'Sullivan, B.J., Ng, L.G., Roediger, B., Thomas, R., Weninger, W., and Fazekas de St Groth, B. (2011). Langerhans cells are precommitted to immune tolerance induction. Proc Natl Acad Sci U S A 108, 18049-18054.

Sichien, D., Lambrecht, B.N., Guilliams, M., and Scott, C.L. (2017). Development of conventional dendritic cells: from common bone marrow progenitors to multiple subsets in peripheral tissues. Mucosal Immunol 10, 831-844.

Strid, J., McLean, W.H.I., and Irvine, A.D. (2016). Too Much, Too Little or Just Enough: A Goldilocks Effect for IL-13 and Skin Barrier Regulation? J Invest Dermatol 136, 561-564.

Stuart, T., Butler, A., Hoffman, P., Hafemeister, C., Papalexi, E., Mauck, W.M., 3rd, Hao, Y., Stoeckius, M., Smibert, P., and Satija, R. (2019). Comprehensive Integration of Single-Cell Data. Cell 177, 1888-1902 e1821.

Subramanian, A., Tamayo, P., Mootha, V.K., Mukherjee, S., Ebert, B.L., Gillette, M.A., Paulovich, A., Pomeroy, S.L., Golub, T.R., Lander, E.S., et al. (2005). Gene set enrichment analysis: a knowledge-based approach for interpreting genome-wide expression profiles. Proc Natl Acad Sci U S A 102, 15545-15550.

Teunissen, M.B.M., Munneke, J.M., Bernink, J.H., Spuls, P.I., Res, P.C.M., Te Velde, A., Cheuk, S., Brouwer, M.W.D., Menting, S.P., Eidsmo, L., et al. (2014). Composition of innate lymphoid cell subsets in the human skin: enrichment of NCR(+) ILC3 in lesional skin and blood of psoriasis patients. J Invest Dermatol 134, 2351-2360.

Tordesillas, L., Lozano-Ojalvo, D., Dunkin, D., Mondoulet, L., Agudo, J., Merad, M., Sampson, H.A., and Berin, M.C. (2018). PDL2(+) CD11b(+) dermal dendritic cells capture topical antigen through hair follicles to prime LAP(+) Tregs. Nat Commun 9, 5238.

Tussiwand, R., Everts, B., Grajales-Reyes, G.E., Kretzer, N.M., Iwata, A., Bagaitkar, J., Wu, X., Wong, R., Anderson, D.A., Murphy, T.L., et al. (2015). Klf4 expression in conventional dendritic cells is required for T helper 2 cell responses. Immunity 42, 916-928.

Wills-Karp, M., and Finkelman, F.D. (2008). Untangling the complex web of IL-4- and IL-13mediated signaling pathways. Sci Signal 1, pe55.

Wojno, E.D., Monticelli, L.A., Tran, S.V., Alenghat, T., Osborne, L.C., Thome, J.J., Willis, C., Budelsky, A., Farber, D.L., and Artis, D. (2015). The prostaglandin D(2) receptor CRTH2 regulates accumulation of group 2 innate lymphoid cells in the inflamed lung. Mucosal Immunol 8, 1313-1323.

Wynn, T.A. (2003). IL-13 effector functions. Annu Rev Immunol 21, 425-456.

Yu, C.I., Becker, C., Wang, Y., Marches, F., Helft, J., Leboeuf, M., Anguiano, E., Pourpe, S., Goller, K., Pascual, V., et al. (2013). Human CD1c+ dendritic cells drive the differentiation of CD103+ CD8+ mucosal effector T cells via the cytokine TGF-beta. Immunity 38, 818-830.

Zeng, R., Bscheider, M., Lahl, K., Lee, M., and Butcher, E.C. (2016). Generation and transcriptional programming of intestinal dendritic cells: essential role of retinoic acid. Mucosal Immunol 9, 183-193.

Ziegler, S.F. (2012). Thymic stromal lymphopoietin and allergic disease. J Allergy Clin Immunol 130, 845-852. 
Figures \& Legends

A

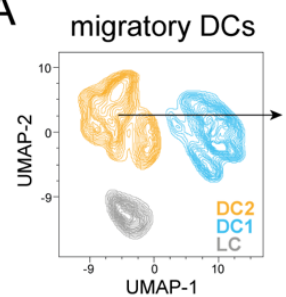

C

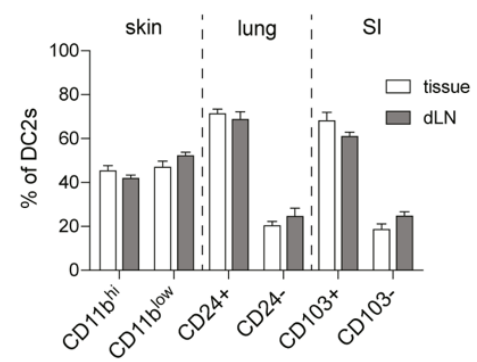

$\mathrm{F}$
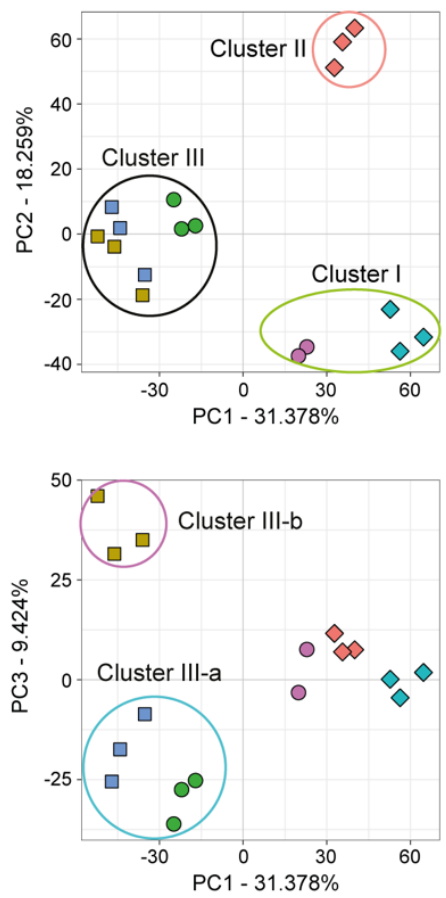

DC2 subsets

Skin-dLN Lung-dLN SI-dLN

$\square \mathrm{CD} 11 \mathrm{~b}^{\mathrm{hi}}$ OCD24+ $\triangle \mathrm{CD} 103+$

$\square$ CD11blow $O$ CD24- $\triangle$ CD103-
B

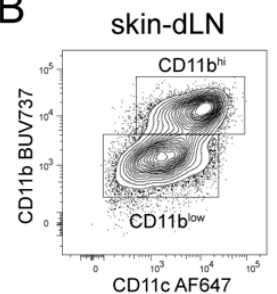

D

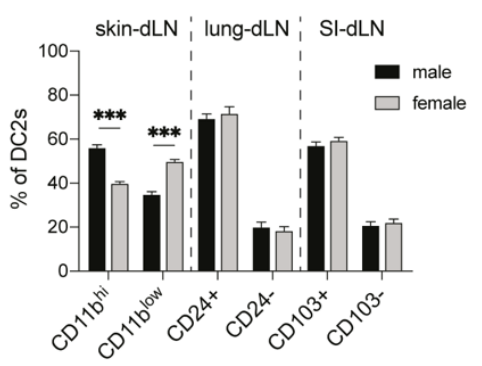

SI-dLN

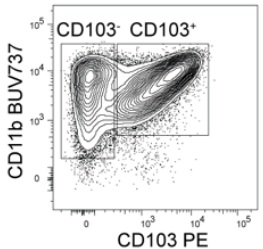

E

G
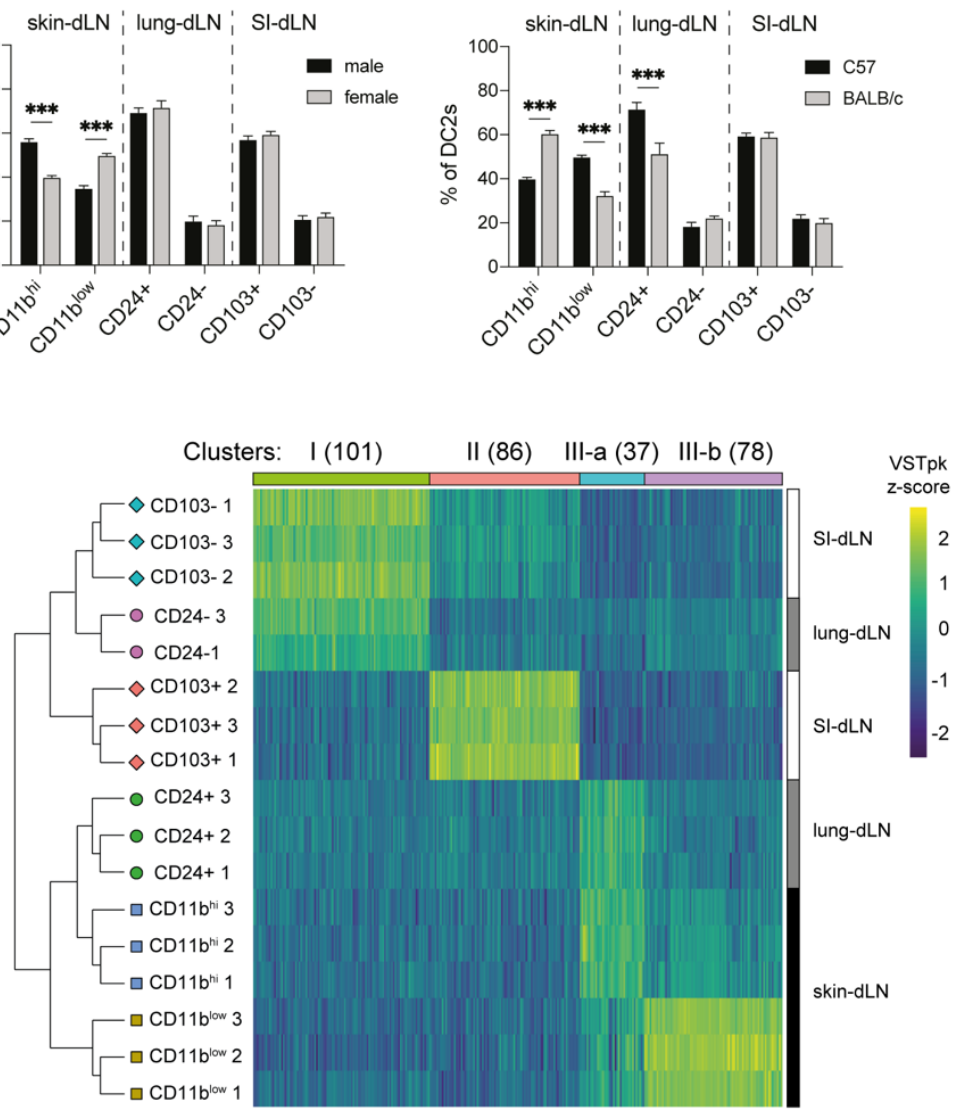

$\mathrm{H}$

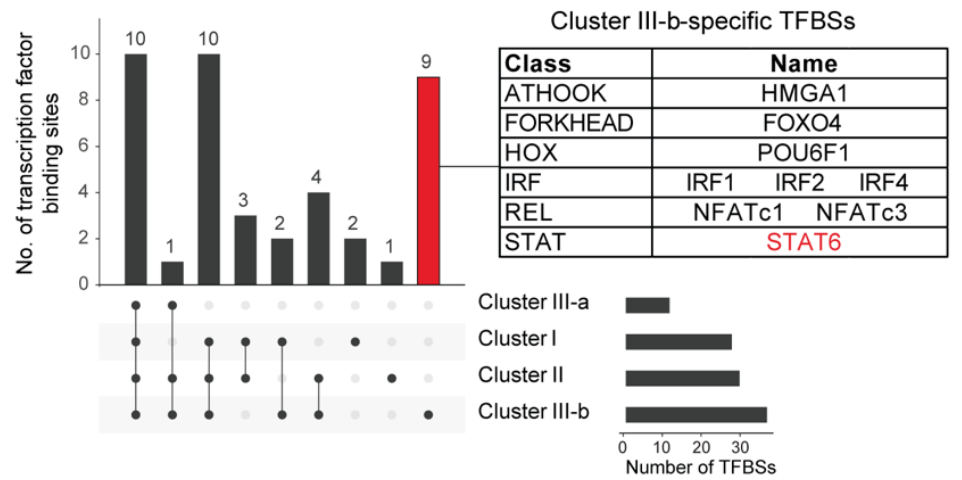

Figure 1. DC2s display tissue and subset-specific heterogeneity at steady state.

(A) UMAP visualization of concatenated migratory dendritic cells (DCs) and DC2s from the skin, lung and small intestine (SI)-draining lymph nodes (dLN) of naïve C57BL/6 (C57) mice. 
DCs were gated as shown in Figure S1. UMAP was performed on 150,000 events $(10,000$ events/tissue/mouse, 5 mice per group) using 7 parameters (XCR1, CD326, Sirp $\alpha$, CD11c, CD11b, CD24 and CD103) with default FlowJo settings of nearest neighbors $=15$ and minimum distance $=0.5$ using the Euclidean distance function .

(B) Representative contour plots displaying DC2 subsets in the skin, lung and SI-dLN.

(C) Relative proportions of DC2 subsets within skin, lung and SI of naïve C57 mice compared to their respective dLNs. Bar graphs show mean \pm SEM; each bar refers to 8-10 mice pooled from 2-3 independent experiments.

(D) Relative proportion of DC2 subsets in the skin, lung and SI-dLN of naïve male and female C57 mice. Bar graphs show mean \pm SEM; each bar refers to 6-8 mice pooled from 2 independent experiments.

(E) Relative proportion of DC2 subsets in the skin, lung and SI-dLN of naïve $\mathrm{C} 57$ and $\mathrm{BALB} / \mathrm{cByJ}(\mathrm{BALB} / \mathrm{c})$ female mice. Bar graphs show mean $\pm \mathrm{SEM}$; each bar refers to 6-8 mice pooled from 2 independent experiments.

(C-E) $P$ values were determined using a two-tailed Student's t-test. ${ }^{* * *} \mathrm{p}<0.001$; only significant comparisons are indicated.

(F) Principal component (PC) analysis of all expressed genes in sorted DC2 subsets from the skin, lung and SI-dLNs of naïve C57 mice reveals three main clusters in PC1 vs. PC2 and two subclusters in PC1 vs. PC3.

(G) Heatmap of differentially expressed genes for each of the clusters and subclusters identified in (F). The number of genes in each cluster and subcluster is shown in parentheses at the top. Inclusion criteria were: protein coding genes; mean normalized RNA count > 20; fold-change greater than 2 and $p<0.05$ as compared to each of the other clusters. VSTpk: Variance-stabilized transformed count per kbp; z-Scores for each gene were calculated using R.

(H) UpSet plot showing the numbers of transcription factor binding sites (TFBSs) that were identified by TRANSFAC promoter analysis in each of the gene clusters in $(G)$. The numbers of TFBSs shared across several clusters (left) or unique to individual clusters (right) are shown. The number of TFBSs that are uniquely enriched in Cluster III-b-specific genes are indicated by the red bar with the TFBSs listed in the adjacent table. 
A
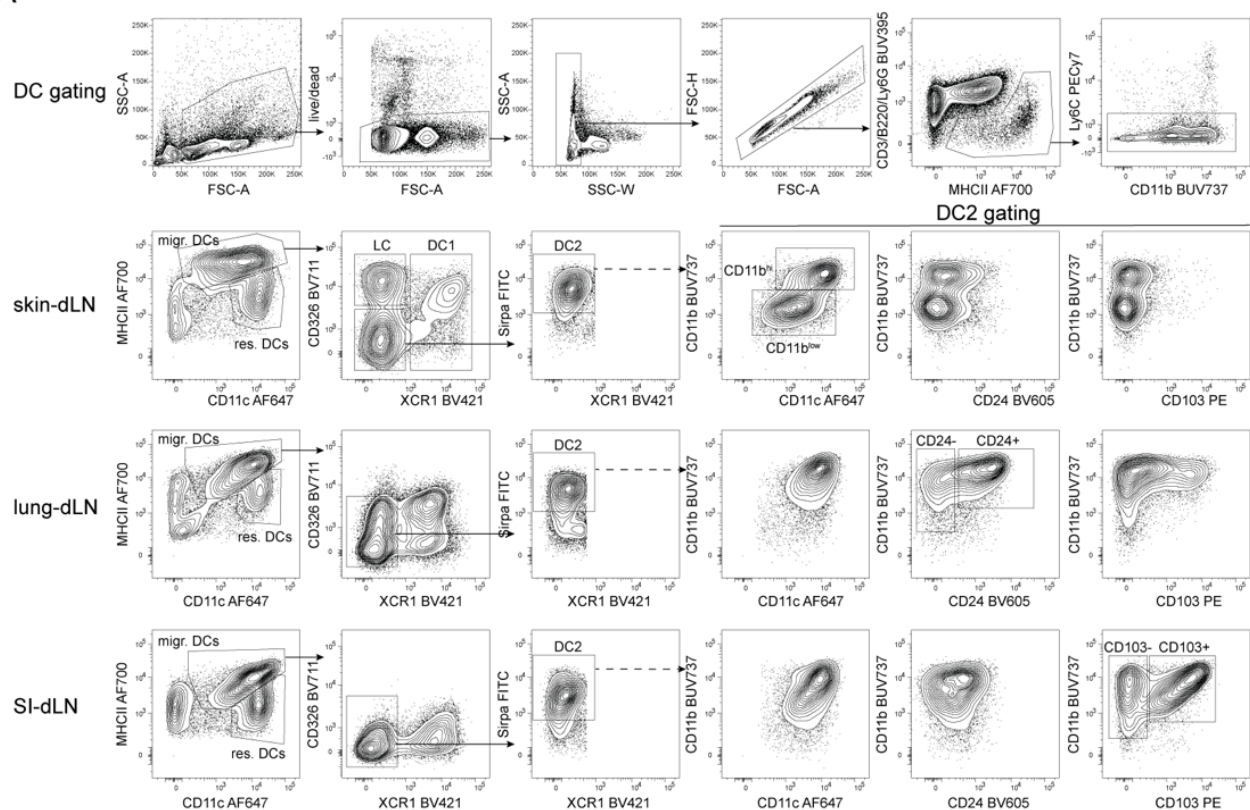

B
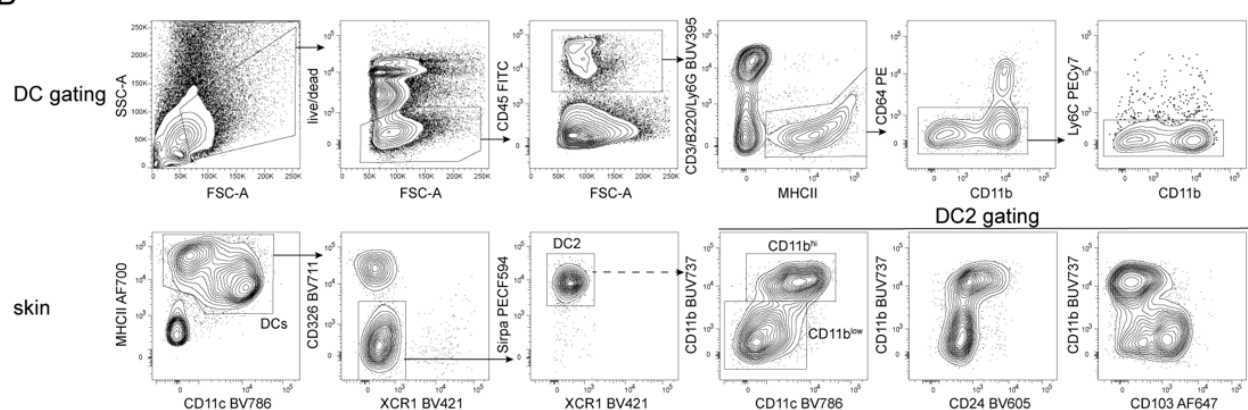

ung
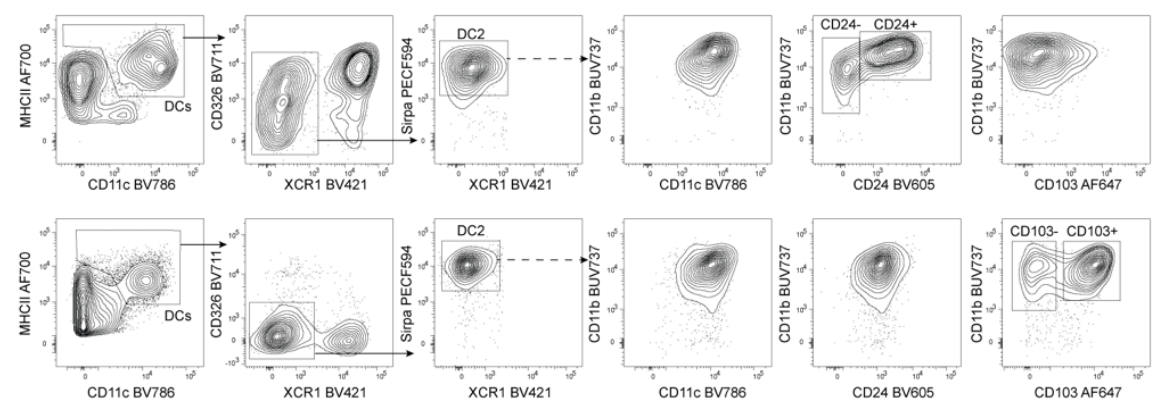

C

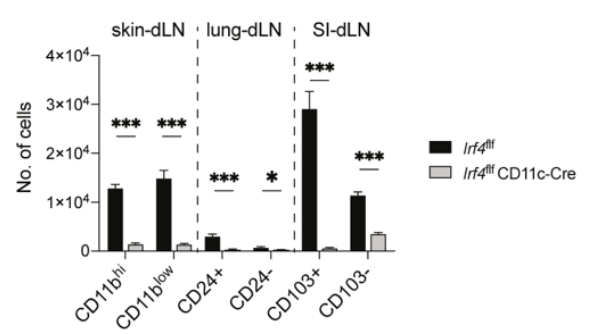

Figure S1. Related to Figure 1: Gating strategy for DC and DC2 populations in the skin, lung and small intestine (SI) of naïve mice and their corresponding draining lymph nodes (dLNs).

(A) Gating strategy for migratory DC2 subsets in dLNs. Generic gating on live single CD3B220- Ly6G $^{-}$Ly6C $^{-}$cells was performed for all samples before gating on dLN-specific DC2 subsets. 
(B) Gating strategy for DC2 subsets in tissues. Generic gating on live single CD45 ${ }^{+} \mathrm{CD}^{-}{ }^{-} \mathrm{B}^{-2} \mathrm{C}^{-}$

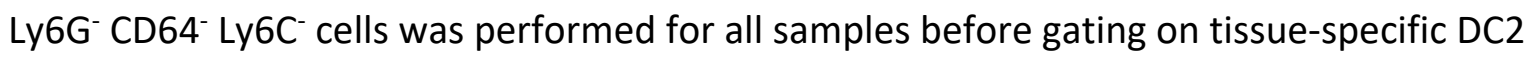
subsets.

(C) Numbers of DC2s in the skin, lung and SI-dLN of Irff ${ }^{f / f}$ and $/ r f 4^{f / f}$ CD11c-Cre mice. Bar graphs show mean \pm SEM, each bar refers to 5-8 mice pooled from 2 independent experiments. $P$ values were determined using a two-tailed Student's t-test. ${ }^{*} p<0.05 ; * * * p$ $<0.001$; only significant comparisons are indicated. 
A bone marrow DC2 precursors
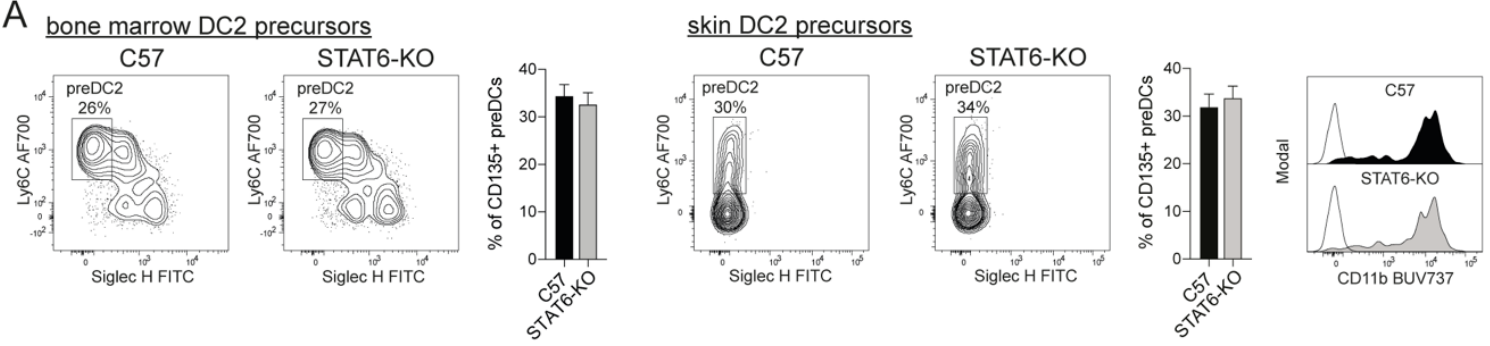

B $\underline{\mathrm{C} 57}$

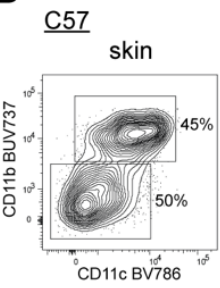

Tissues
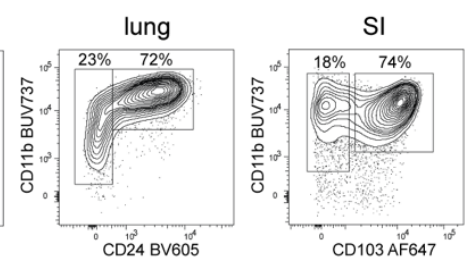

$\mathrm{D}$

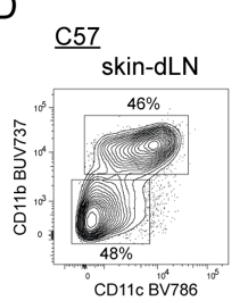

Lymph nodes
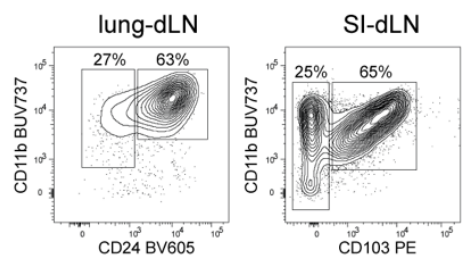

$\underline{\text { STAT6-KO }}$
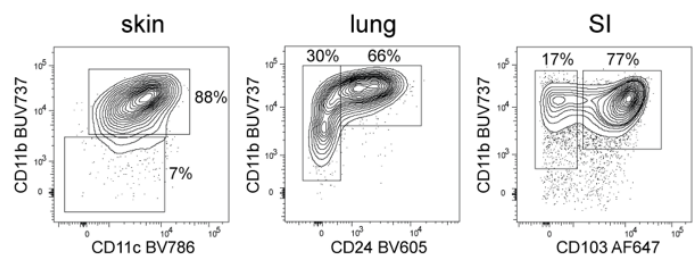

STAT6-KO
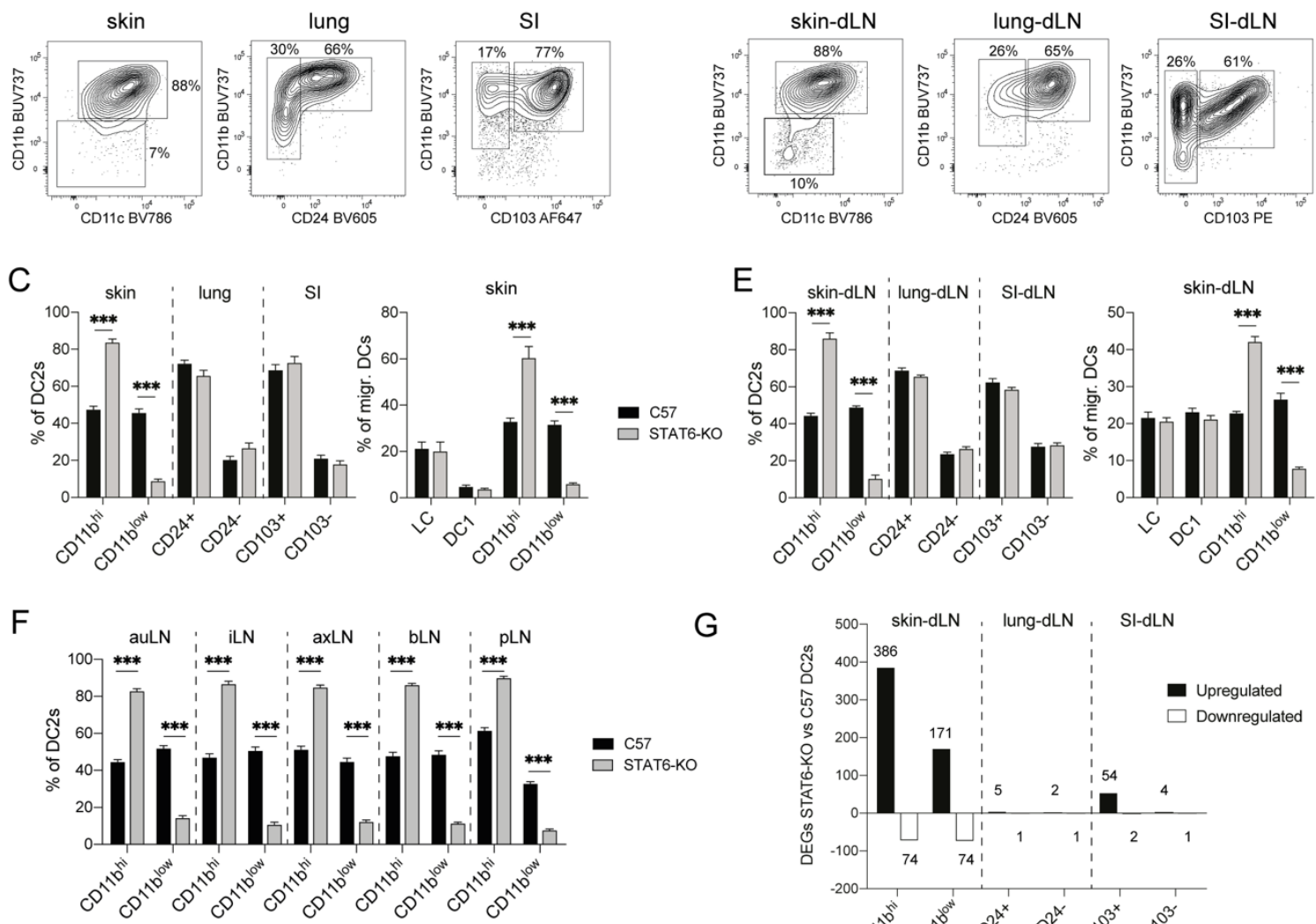

G

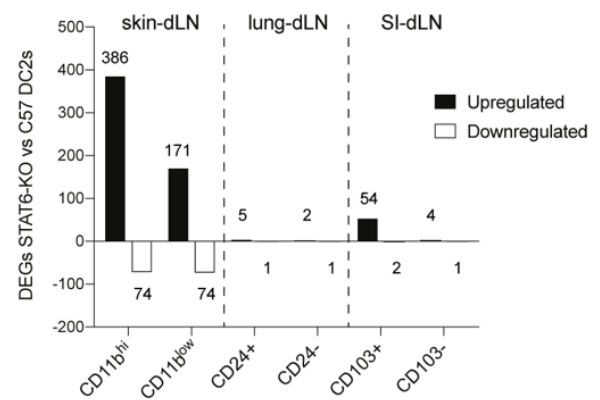

Figure 2. STAT6-dependent signalling controls DC2 development in the skin at steady state.

(A) Representative contour plots showing the phenotype and frequency of preDC populations in the bone marrow and skin of naïve C57 and STAT6-KO mice, as gated in Figure S2A; preDC2 are identified by the gates and are $\mathrm{Ly} 6 \mathrm{C}+\mathrm{Siglec} \mathrm{H}^{\text {low }} \mathrm{B} 220^{\text {low }}$. Histograms on the right show CD11b expression on skin preDC2s from C57 and STAT6-KO mice (filled histograms) compared to FMO (empty histograms). Representative contour plots and histograms show concatenated data from 4-5 mice. Bar graphs show mean \pm SEM; each bar refers to 6 mice (BM) or 9-10 mice (skin) pooled from 2 independent experiments. $P=0.62$ (BM) and 0.61 (ear) were determined using a two-tailed Student's t-test. 
(B) Representative contour plots showing the phenotype and relative frequencies of DC2 subsets in the skin, lung and small intestine (SI) of naïve C57 and STAT6-KO mice.

(C) Relative frequencies of DC2 subsets in the skin, lung and SI (left) and frequencies of DC subsets in the skin (right) of naïve C57 and STAT6-KO mice. Bar graphs show mean \pm SEM, each bar refers to 7-11 mice pooled from 2-3 independent experiments.

(D) Representative contour plots showing the phenotype of DC2 subsets in the skin, lung and SI-draining lymph nodes (dLNs) of naïve C57 and STAT6-KO mice.

(E) Relative frequencies of migratory DC2 subsets in the skin, lung and SI-dLNs (left) and frequencies of migratory DC subsets in the skin-dLNs (right) of naïve C57 and STAT6-KO mice. Bar graphs show mean \pm SEM, each bar refers to 4-5 mice (DC2 graph) or 9-10 mice (DC graph) pooled from 2 independent experiments.

(F) Relative frequencies of CD11 $b^{\text {hi }}$ and CD11 $b^{\text {low }}$ DC2 subsets in the auricular (auLN), inguinal (iLN), axillary (axLN), brachial (bLN) and popliteal (pLN) skin-dLNs of naïve C57 and STAT6-KO mice. Bar graphs show mean \pm SEM, each bar refers to 5-8 mice pooled from 2 independent experiments.

(C, E, F) $P$ values were determined using a two-tailed Student's t-test. ${ }^{* *} \mathrm{p}<0.001$; only significant comparisons are indicated.

(G) Number of up- or down-regulated differentially expressed genes (DEGs) in sorted DC2 subsets from skin, lung and SI-dLNs of naïve STAT6-KO vs C57 mice. Protein-coding genes with a mean normalized count $>20$, a fold-change greater than 2 in either direction and a $p$ value $<0.05$ were considered differentially expressed. 
A

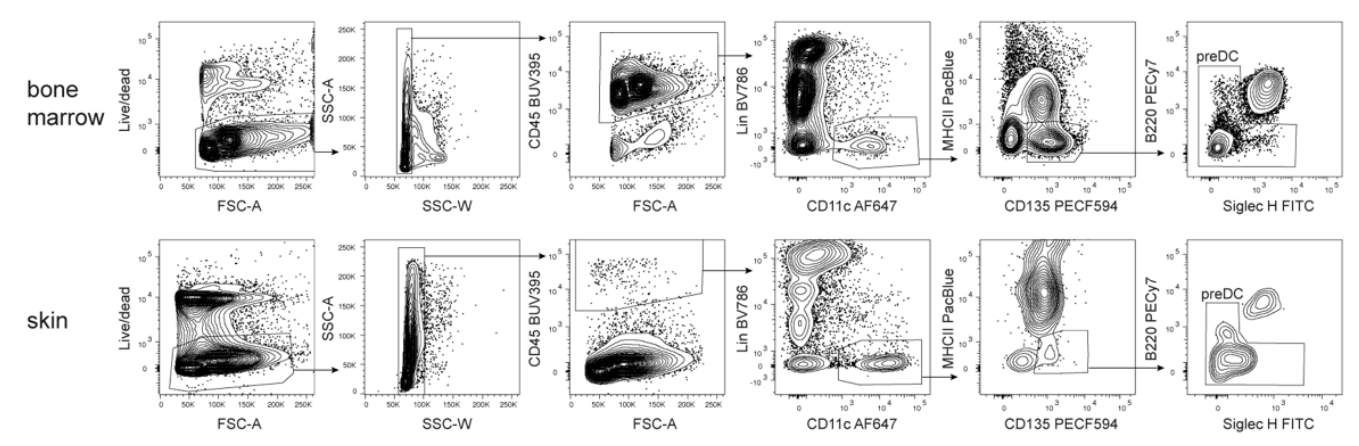

B
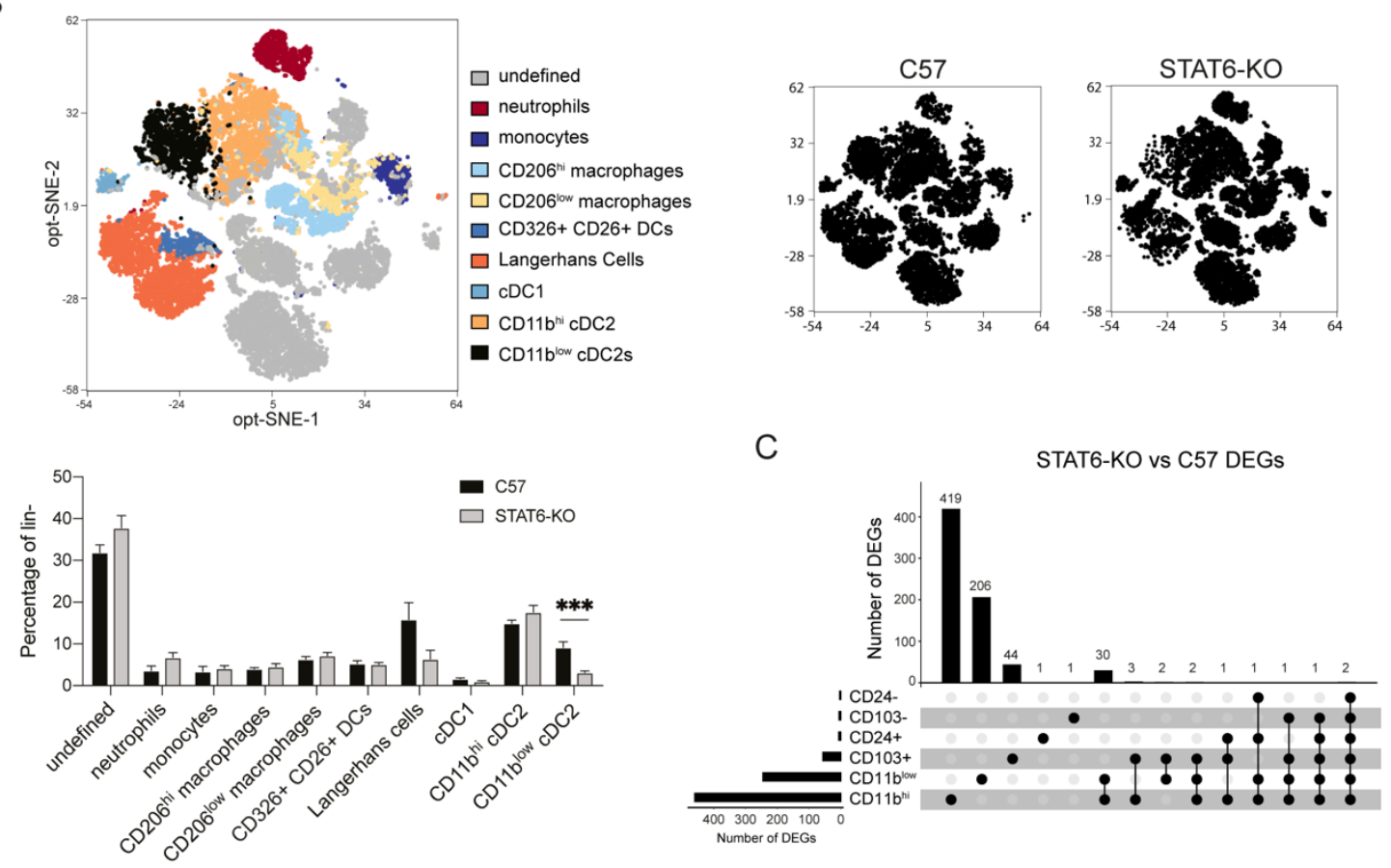

D
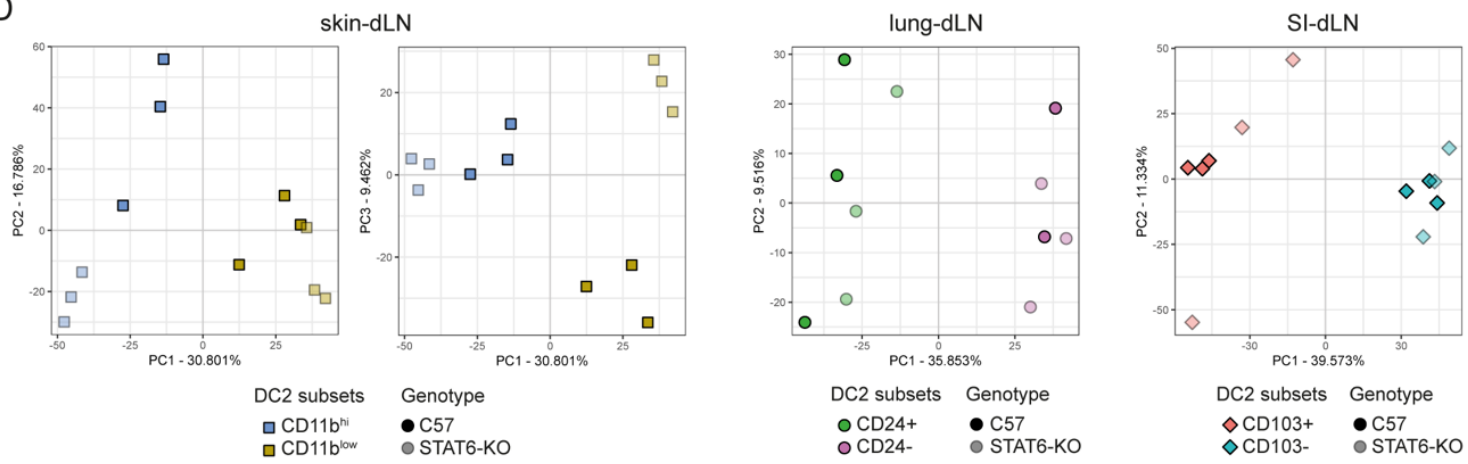

Figure S2. Relating to Figure 2: STAT6 signalling controls a distinct transcriptional program in CD11b ${ }^{\text {hi }}$ and CD11b ${ }^{\text {low }}$ DC2s.

(A) Gating strategy for preDC2s in the bone marrow (upper panels) and skin (lower panels). 
(B) Representative tsne visualization of concatenated live single $\mathrm{CD}_{4}{ }^{+} \mathrm{CD} 3^{-} \mathrm{CD} 19^{-}$myeloid cells from the skin of naïve C57BL/6 (C57) and STAT6-KO male mice; combined data from both strains are shown in the color image.

Opt-SNE was performed on 16648 events (2081 events/mouse, 4 mice per genoype) using 12 parameters (XCR1, CD206, CD11b, Ly6c, CD11c, Ly6G, SIRP $\alpha$, CD326, CD26, CD64, BST2, MHC-II) with default OMIQ settings and a perplexity of 30 with 1000 iterations. The bar graph shows mean \pm SEM, each bar refers to 9 mice pooled from 2 independent experiments. $P$ values were determined using a two-tailed Student's t-test. ${ }^{* * *} p<0.001$; only significant comparisons are indicated.

(C) UpSet plot showing the number of unique and shared differentially expressed genes (DEGs, including up- and down-regulated) in the indicated DC2 subsets sorted from the skin, lung and SI-draining lymph nodes (dLNs) of STAT6-KO and C57 mice using the gating in S1A.

(D) Principal component (PC) analyses of all expressed genes in sorted DC2 subsets from the skin (upper panels), lung and SI (lower panels) -dLNs of naïve C57 and STAT6-KO mice. 
A

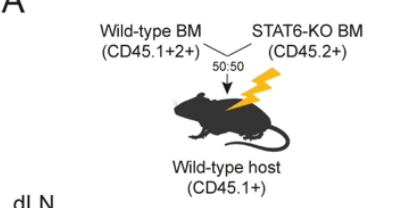

dLN

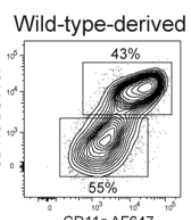

STAT6-KO-derived

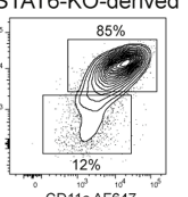

D

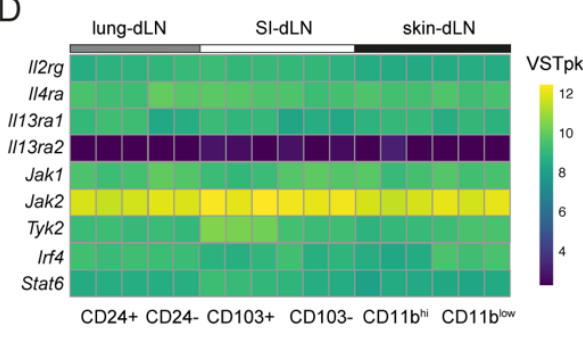

$\mathrm{F}$
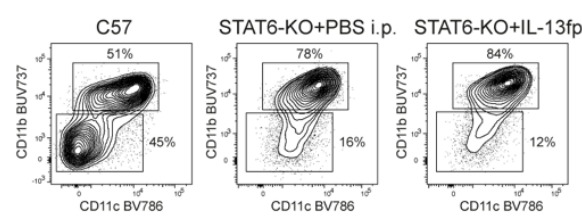

G

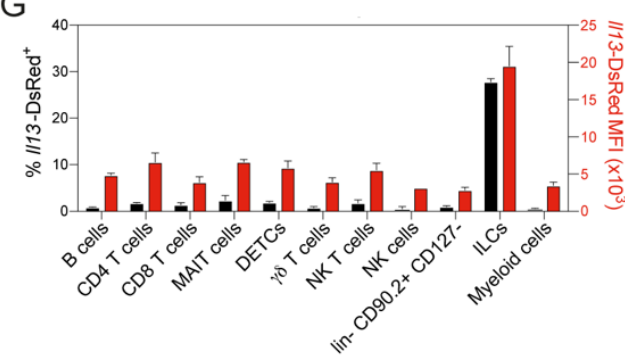

$\mathrm{J}$
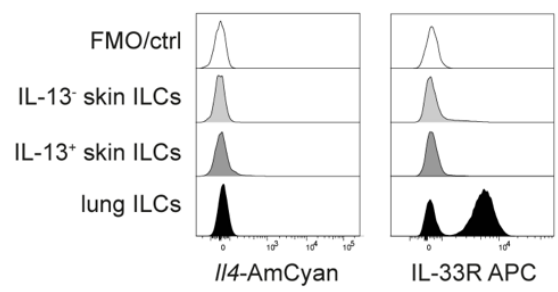

IL-33R APC
B
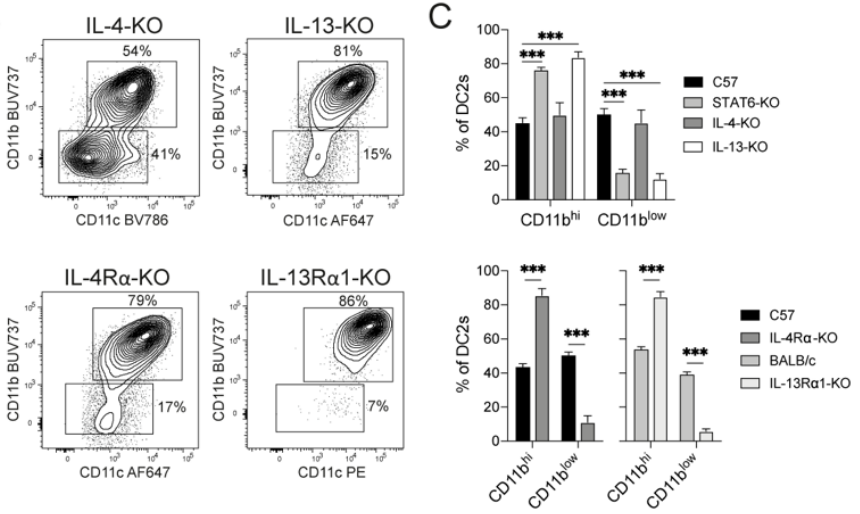

E

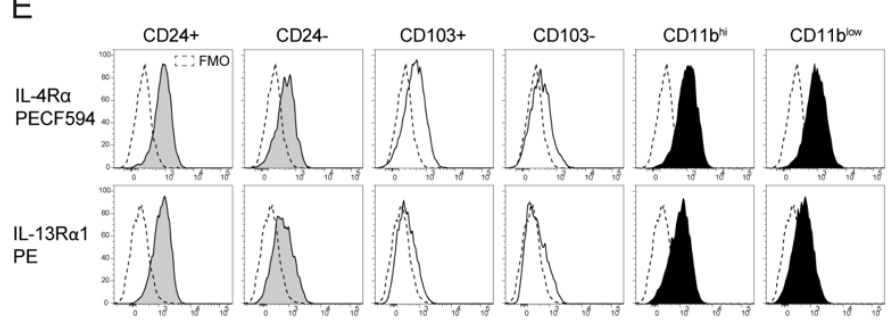

Figure 3. IL-13 is necessary and sufficient for the development of dermal CD11b ${ }^{\text {low }}$ DC2s and is expressed by skin innate cells at steady state.

(A) Experimental set-up of mixed wild-type and STAT6-KO bone marrow (BM) chimeras. Representative contour plots show the phenotype and relative frequencies of skin-draining lymph node (dLN) DC2s in each donor BM. The bar graph shows mean \pm SEM, each bar refers to 8 mice pooled from 2 independent experiments. $P$ values were determined using a two-tailed Student's t-test. ${ }^{* * *} \mathrm{p}<0.001$. 
(B) Representative contour plots showing the phenotype and relative frequencies of skin$\mathrm{dLN}$ DC2 subsets in naïve mice of the indicated strain. All KO strains were on a C57BL/6 (C57) background except for the IL-13R $\alpha 1-K O$ which was on a BALB/c background.

(C) Relative frequencies of skin-dLN DC2s in naïve mice of the indicated strains. Bar graphs show mean \pm SEM, each bar refers to 6-10 mice pooled from 2 independent experiments. $P$ values were determined using two-way ANOVA with Sidak correction. ${ }^{* * *} p<0.001$; only significant comparisons are indicated.

(D) Heatmap showing Variance-stabilized and transformed read counts/kbp (VSTpk) for transcripts associated with the IL-4 and IL-13 signaling pathway as assessed in sorted DC2 subsets from the lung, skin and SI-dLNs of C57 mice.

(E) IL-4R $\alpha$ and IL-13R $\alpha 1$ expression on DC2 subsets from the lung, skin and SI-dLNs of C57 mice as determined by fluorescent staining and flow cytometry. Dashed lines show FMOs. Histograms show concatenated data from 4 mice in one of two independent experiments that gave similar results.

(F) Representative contour plots showing the phenotype and relative frequencies of skindLN DC2s in C57 controls and in mutant mice of the indicated strains that were treated with either PBS or IL-13 fusion protein (IL-13fp) for 4 days before analysis. The bar graph shows mean \pm SEM, each bar refers to 3-6 mice pooled from 1-2 independent experiments. $P$ values were determined using two-way ANOVA with Sidak correction. $* * * p<0.001$; only relevant significant comparisons are indicated.

(G) Frequencies of //13-DsRed ${ }^{+}$cells and //13-DsRed Median Fluorescence Intensities (MFI) within different populations of innate and adaptive immune cells in the ear skin of naïve 4 C13R reporter mice. Bar graph shows mean \pm SEM, each bar refers to 6 pools of 3 mice from 2 independent experiments. $P$ values were determined using one-way ANOVA with Sidak correction. ${ }^{* * *} \mathrm{p}<0.001$; only significant comparisons are indicated.

(H) Pie chart showing the proportion of different immune cell populations within the /I13DsRed $^{+}$population in the ear skin of naïve 4C13R reporter mice. Data are from (G).

(I) Frequencies of II13-DsRed ${ }^{+}$cells within the innate lymphoid cell (ILC) population in skin, lung and SI of naïve 4C13R reporter mice. Bar graph shows mean \pm SEM, each bar refers to 6-7 mice pooled from 2 independent experiments. $P$ values were determined using an ordinary one-way ANOVA with Sidak correction. ${ }^{* * *} p<0.001$; only significant comparisons are indicated.

(J) Expression of //4-AmCyan and ILC surface markers in I/13-DsRed-negative or -positive ILCs from the skin or lung of naïve 4C13R reporter mice. A non-reporter mice was used as II4AmCyan fluorescence-minus-one (FMO) control. Histograms show concatenated data from 4 mice in one of 4 independent experiments that gave similar results. 
A

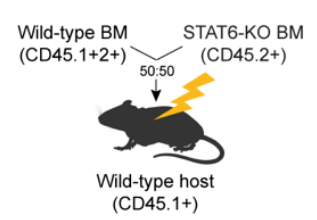

B

Genomic locus of murine $/ 113$

WT allele

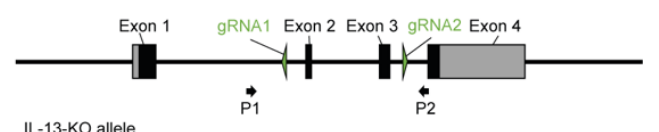

IL-13-KO allele

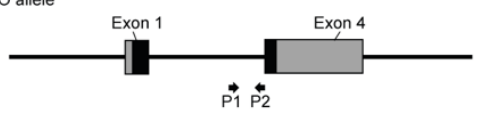

E Gating of lymphoid populations
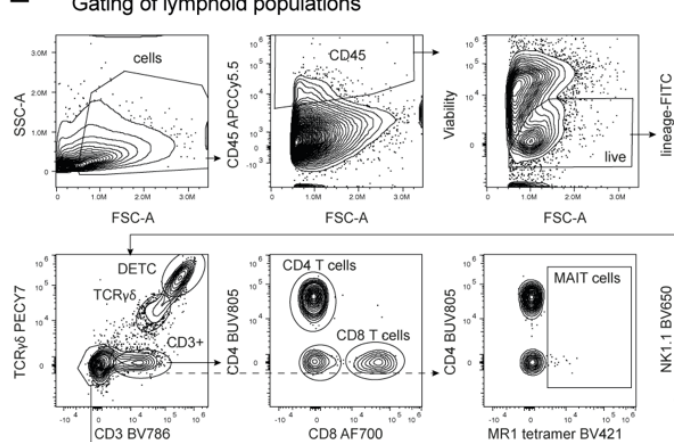

CD3 BV786

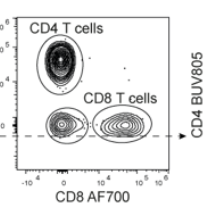

CD8 AFF00
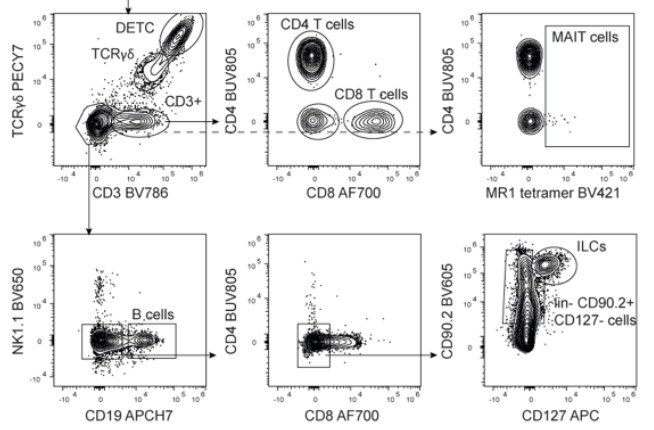

MR1 tetramer BV421

G
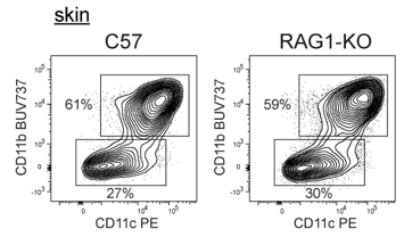

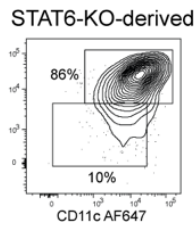

C
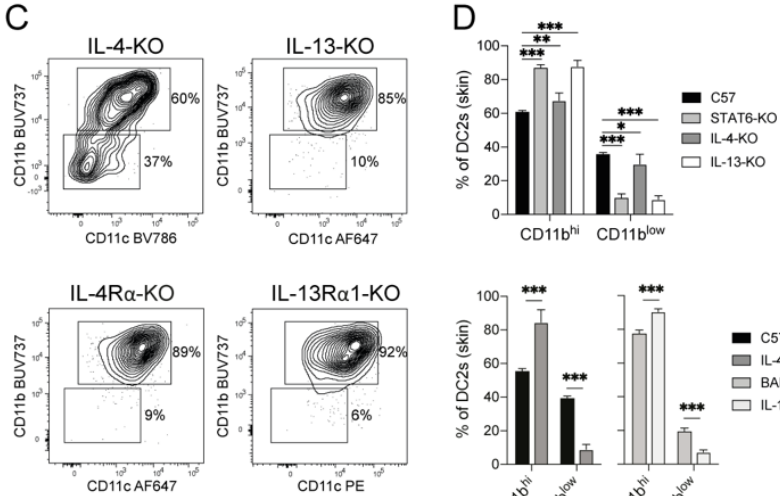

F ILC gating (skin)

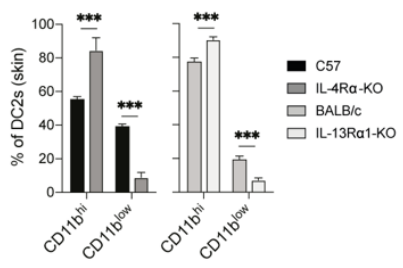

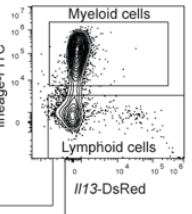
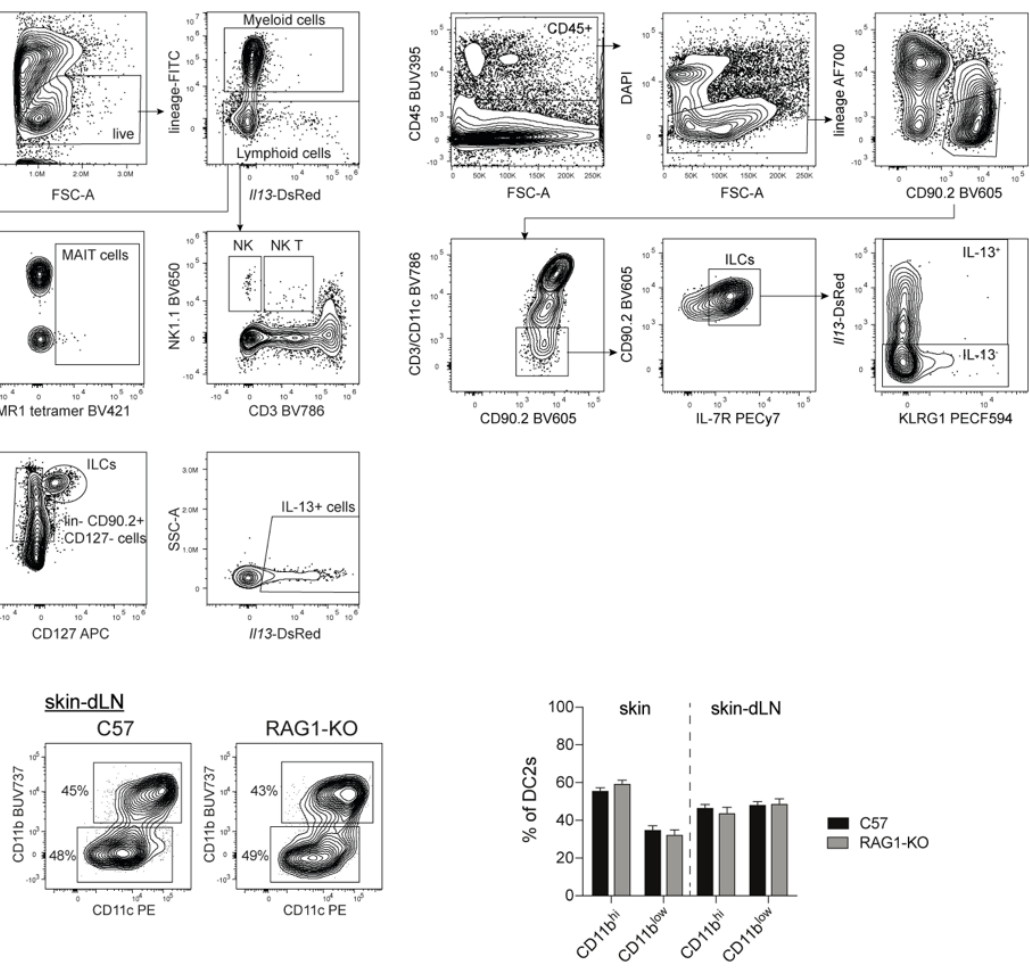

Figure S3. Related to Figure 3: IL-13 signalling is necessary for the development of CD11 $b^{\text {low }}$ DC2s in skin and is independent of adaptive or KLRG1+ cell popoulations

(A) Experimental set-up of the mixed wild-type and STAT6-KO BM chimeras in Figure 3A-C. Representative contour plots show the phenotype and relative frequencies of skin DC2s from each donor BM. The bar graph shows mean \pm SEM, each bar refers to 8 mice pooled from 2 independent experiments. $P$ values were determined using a two-tailed Student's ttest. $* * * p<0.001$.

(B) Schematic of the genomic I/13 locus of IL-13-KO mice illustrating the deletion of exons 2 and 3. 
(C) Representative contour plots showing the phenotype and relative frequencies of skin DC2s in naïve mice of the indicated strains. All KO strains were on a C57BL/6 background except for the IL-13R $\alpha 1-K O$ which were on a BALB/c background.

(D) Relative frequencies of skin DC2 subsets in naïve mice of the indicated strains. Bar graphs show mean \pm SEM, each bar refers to 5-15 mice pooled from 2-3 independent experiments. $P$ values were determined using two-way ANOVA with Sidak correction. ${ }^{*} p<$ $0.05 ; * *<0.01 ; * * p<0.001$.

(E) Gating strategy to identify lymphoid cell populations in the ear skin of naïve 4C13R reporter mice. To define cells of lymphoid origin, cells were pre-gated on single, live, $C D 45^{+}$,

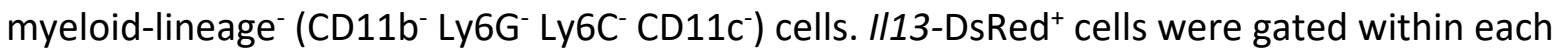
cell population, or in the total $C D 45^{+}$population as shown in the lower right panel.

(F) Gating strategy to identify innate lymphoid cells (ILCS) in the skin of naïve 4C13R reporter mice. A similar gating strategy was used to identify ILCs in lung and small intestine.

(G) Representative contour plots showing the phenotype and relative frequencies of skin and skin-draining lymph node (dLN) DC2 subsets in naïve C57BL/6 (C57) and RAG1-KO mice. Bar graph shows mean \pm SEM, each bar refers to 8-10 mice pooled from 2 independent experiments. $P$ values were determined using a two-tailed Student's t-test. 
A

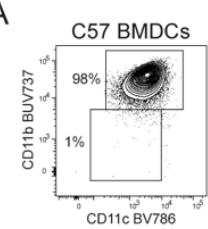

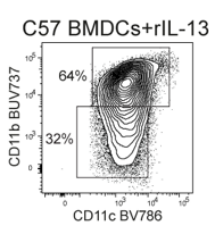
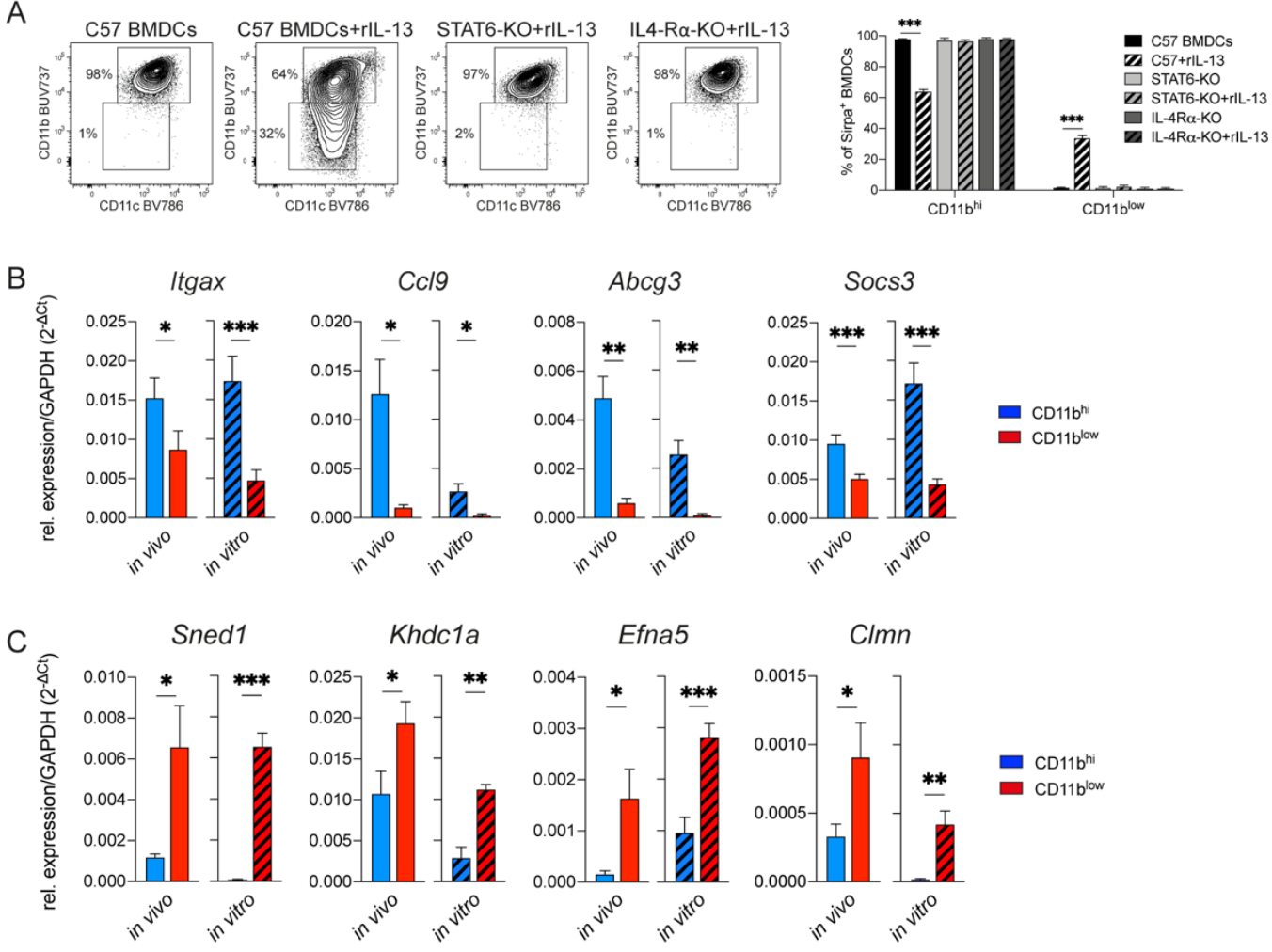

$\mathrm{D}$
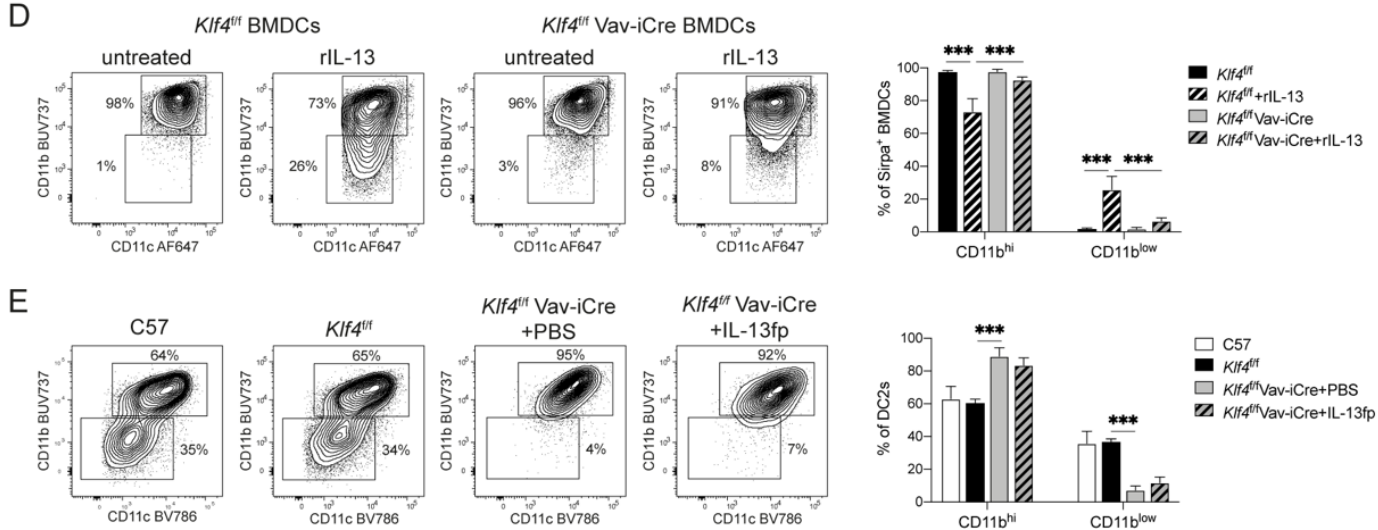

Figure 4. IL-13 signalling drives the development of CD11 b ${ }^{\text {low }}$ DC2 in vitro and is dependent on KLF4 expression in vitro and in vivo.

(A) Representative contour plots showing the phenotype and relative frequencies of CD11 $b^{\text {hi }}$ and CD11b ${ }^{\text {low }}$ Sirpa ${ }^{+}$cells in FLT3L bone marrow (BM)-DC cultures from C57BL/6 (C57) mice or mice of the indicated mouse strains. Cultures were untreated or supplemented with $10 \mathrm{ng} / \mathrm{ml}$ recombinant mouse (r)IL-13 for the last $72 \mathrm{~h}$ of culture. Bar graphs show mean \pm SEM, each bar refers to 7-8 culture wells pooled from 2 independent experiments. $P$ values were determined using a two-way ANOVA with Sidak correction. ${ }^{* * *} p$ $<0.001$; only relevant significant comparisons are indicated.

$(B, C)$ Relative expression of $C D 11 b^{\text {hi }}(B)$ and $C D 11 b^{\text {low }}(C)$ DC2 signature transcripts in sorted DC2 populations from C57 skin-draining lymph nodes (dLN) (in vivo) or C57 FLT3L BMDC cultures that were supplemented with $10 \mathrm{ng} / \mathrm{ml} \mathrm{rlL-13}$ for the last $72 \mathrm{~h}$ (in vitro). Transcript levels were measured by RT-qPCR. Bar graphs show mean \pm SEM, each bar refers to 6 mice 
(in vivo) or 8 cultures (in vitro) pooled from 2 independent experiments. $P$ values were determined using a paired, two-tailed Student's t-test. ${ }^{*} p<0.05 ;{ }^{* *} p<0.01 ; * * p<0.001$.

(D) Representative contour plots showing phenotype and relative frequencies of CD11 $\mathrm{b}^{\text {hi }}$ and CD11 bow Sirpa ${ }^{+}$FLT3L BMDCs in cultures of BM cells from $\mathrm{Klfl}^{\mathrm{f} / \mathrm{f}} \rightarrow \mathrm{C} 57 \mathrm{BL} / 6$ or Klf4 $4^{\mathrm{f} / \mathrm{f}}$ Vav-iCre $\rightarrow$ C57BL/6 male chimeric mice. Cultured cells were untreated or supplemented with $10 \mathrm{ng} / \mathrm{ml} \mathrm{rlL}-13$ for the last $72 \mathrm{~h}$ of culture. Bar graphs show mean $\pm \mathrm{SEM}$, each bar refers to 4 cultures pooled from 3 independent experiments. $P$ values were determined using two-way ANOVA with Sidak correction. ${ }^{* * *} p<0.001$; only relevant significant comparisons are indicated.

(E) Representative contour plots showing the phenotype and relative frequencies of DC2 subsets in the skin-dLNs of intact C57 mice or chimeric Klf4 $4^{f / f} \rightarrow C 57 B L / 6$ or Klf4 ${ }^{f / f}$ Vav-iCre $\rightarrow$ C57BL/6 mice. Mice were treated with PBS or IL-13 fusion protein (IL-13fp) for 4 days as indicated. Bar graphs show mean \pm SEM, each bar refers to 4-9 mice pooled from 2-3 independent experiments. $P$ values were determined using two-way ANOVA with Sidak correction. ${ }^{* * *} p<0.001$; only relevant significant comparisons are indicated. 


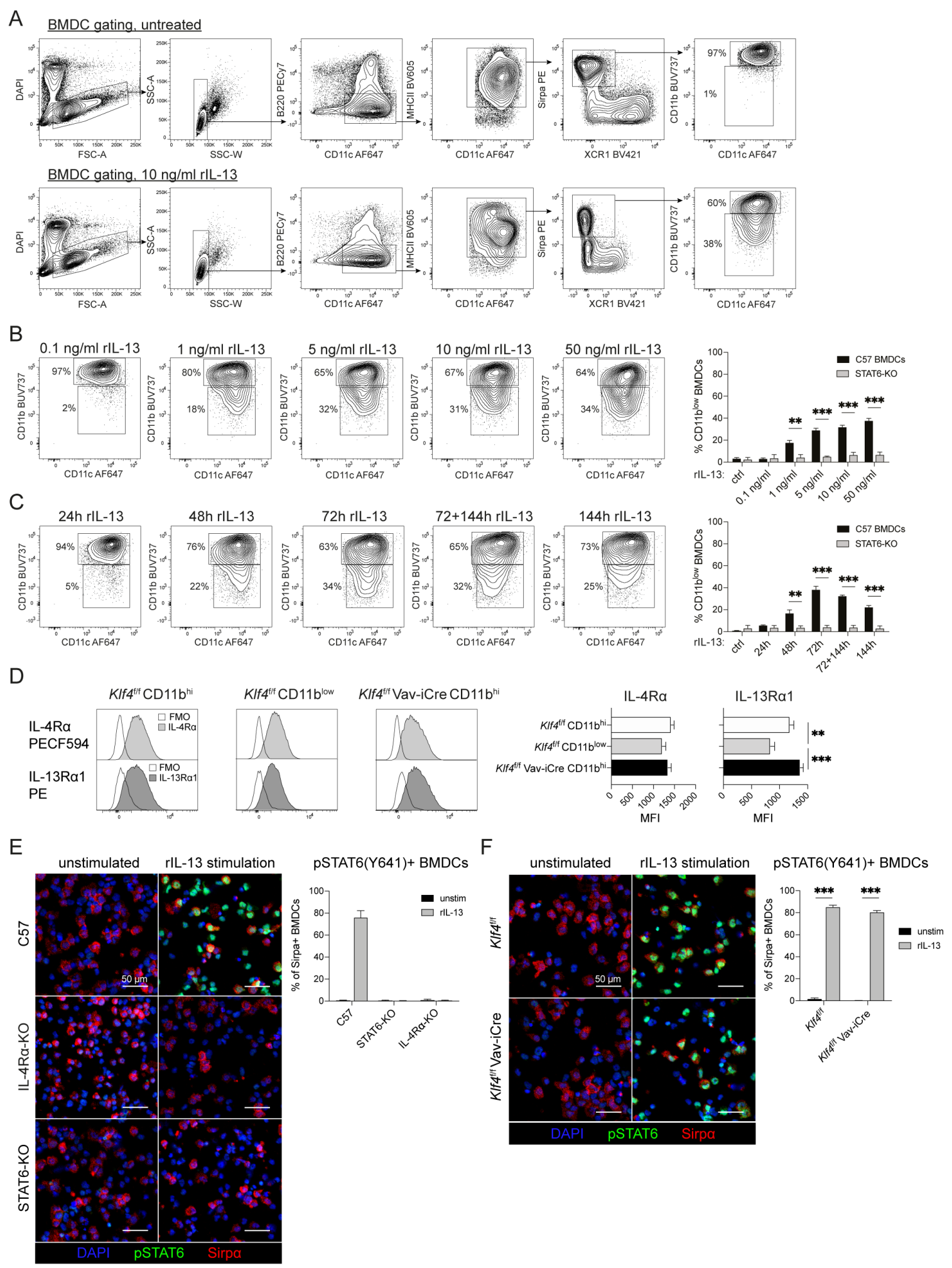

Figure S4. Relating to Figure 4: IL-13 signalling drives the development of CD11b ${ }^{\text {low }}$ DC2 in vitro in a dose- and time-dependent manner.

(A) Gating strategy to identify Sirpa+ DC2 in FLT3L BMDC cultures established from C57BL/6 (C57) BM. Cultures were untreated (upper panels) or supplemented with $10 \mathrm{ng} / \mathrm{ml} \mathrm{rIL-13} \mathrm{for}$ the last $72 \mathrm{~h}$ of culture (lower panels). 
(B) Representative contour plots showing the phenotype and relative frequencies of Sirpa ${ }^{+}$ DC2 subsets in FLT3L BMDCs cultures from C57 BM. Cells were cultured with the indicated concentrations of rlL-13 for the last $72 \mathrm{~h}$ of culture. The bar graph shows mean \pm SEM, each bar refers to 4 cultures pooled from 2 independent experiments. $P$ values were determined using a two-tailed Student's t-test. ${ }^{* *} p<0.01 ;{ }^{* *} p<0.001$; only significant comparisons are indicated.

(C) Representative contour plots showing the phenotype and relative frequencies of Sirpa ${ }^{+}$ DC2 subsets in FLT3L BMDC cultures from C57 BM. Cell cultures were supplemented with $10 \mathrm{ng} / \mathrm{ml} \mathrm{rIL-13}$ at the indicated times before harvest. The bar graph shows mean \pm SEM, each bar refers to 4 cultures pooled from 2 independent experiments. $P$ values were determined using a two-tailed Student's t-test. ${ }^{* *} p<0.01 ; * * p<0.001$; only significant comparisons are indicated.

(D) Representative histograms showing IL-4R $\alpha$ and IL-13R $\alpha 1$ expression on DC2 subsets from the skin-draining lymph nodes of $K I f 4^{f / f} \rightarrow \mathrm{C} 57$ and $K I f 4^{f / f}$ Vav1-iCre $\rightarrow$ C57 chimeric mice as determined by fluorescent staining and flow cytometry. Empty histograms refer to FMOs. Histograms show concatenated data from 3 mice. Bar graphs show mean \pm SEM for 10-11 mice pooled from 3 independent experiments. $P$ values were determined using an ordinary one-way ANOVA with Sidak correction. ${ }^{* *} p<0.01 ;{ }^{* * *} p<0.001$; only significant comparisons are indicated.

(E, F) Representative images of male C57, IL-4R $\alpha-K O$, STAT6-KO (E) Klff ${ }^{\mathrm{f} / \mathrm{f}} \rightarrow \mathrm{C} 57$ and $\mathrm{Klf} 4^{\mathrm{f} / \mathrm{f}}$ Vav1-iCre $\rightarrow$ C57 (F) FLT3L BMDCs that were either unstimulated or treated with $100 \mathrm{ng} / \mathrm{ml}$ rIL-13 for 30 minutes, centrifuged on a glass slide, fixed and stained with anti-Sirpa, antiphospho-STAT6 (Y641) and DAPI. Scale bars correspond to $50 \mu \mathrm{m}$. An average of 2200 Sirp $\alpha+$ cells/sample were assessed. Bar graphs show the mean frequency of pSTAT6+ cells in the Sirp $\alpha+B M D C$ population \pm range $(E)$ or \pm SEM $(F)$, each bar refers to $2(E)$ or 5-6 (F) cultures pooled from 2 separate experiments. $P$ values were determined using multiple $t$ tests. ${ }^{* *} \mathrm{p}<0.001$; only significant comparisons are indicated. 
A

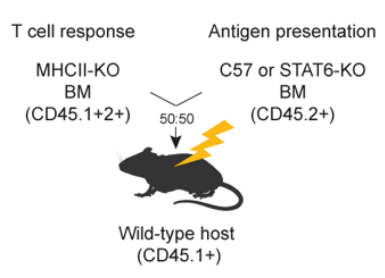

B

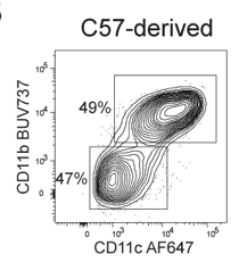

STAT6-KO-derived

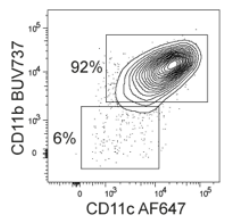

C

D
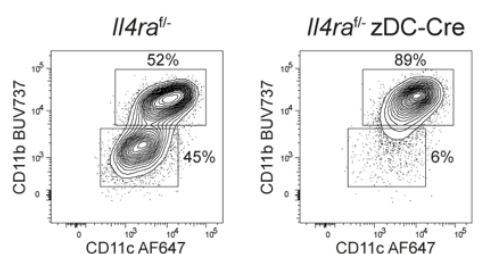

$\mathrm{F}$

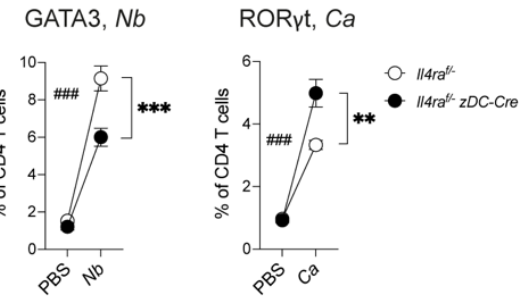

G

total $\mathrm{Nb}-\mathrm{AF} 488$
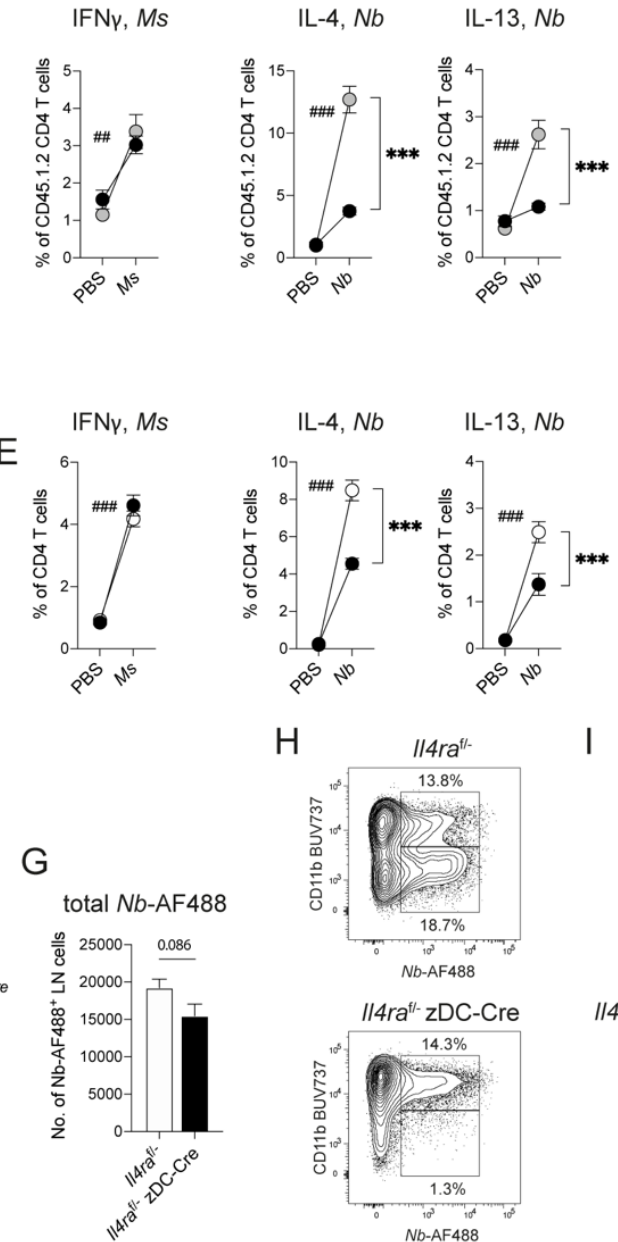

IL-17, Ca
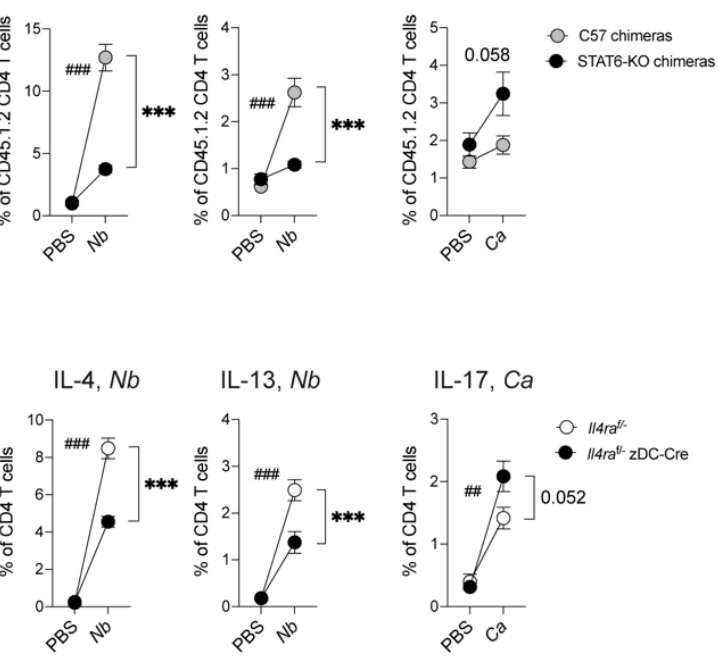

$\mathrm{H}$

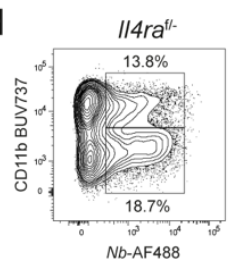

I $\quad 114 \mathrm{ra}^{\mathrm{f} /}, \mathrm{Nb}-\mathrm{AF} 488^{+}$

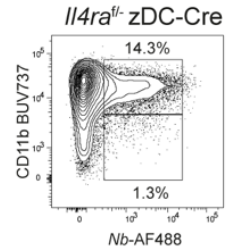

$4 \mathrm{ra}^{\mathrm{f} / \mathrm{z}} \mathrm{zC}-\mathrm{Cre}, \mathrm{Nb}-\mathrm{AF} 488^{+}$

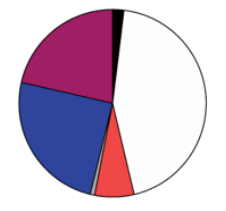

$\mathrm{K}$

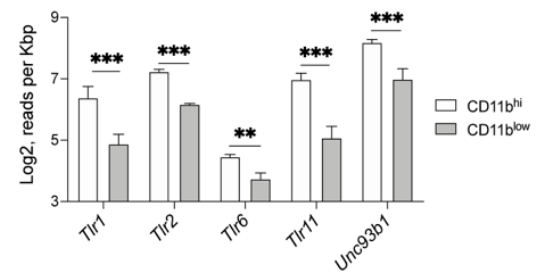

Figure 5. IL-13 signalling in DCs is required for optimal IL-4 ${ }^{+}$and IL-13+ $\mathrm{CD} 4 \mathrm{~T}$ cell responses in skin-draining lymph node (dLN).

(A) Experimental set-up of mixed bone marrow (BM) chimeras in which the antigenpresenting function of $\mathrm{MHClI}^{+} \mathrm{CD} 45.2^{+}$conventional DCs from either $\mathrm{C} 57$ or STAT6-KO BM was assessed by measuring the response of STAT6-sufficient CD45. $1^{+} 2^{+} \mathrm{T}$ cells from $\mathrm{MHCll}-$ KO BM.

(B) Representative contour plots showing phenotype and relative frequencies of C57- or STAT6-KO-derived DC2 subset in the skin-dLN of naïve C57 or STAT6-KO BM chimeras.

(C) Frequency of cytokine-expressing CD45.1 $1^{+} 2^{+}$CD4 T cells in skin-dLNs of C57 or STAT6-KO mixed BM chimeras 5 days after intradermal immunization with either Mycobacterium smegmatis (Ms), Nippostrongylus brasiliensis L3 larvae (Nb), Candida albicans (Ca), or PBS as 
a control. Intracelluar cytokine staining was performed after 5h PMA+ionomycin stimulation. Symbols show mean \pm SEM and refer to 4-6 mice/group pooled from 2 independent experiments. $P$ values were determined using two-way ANOVA with Sidak correction.

(D) Representative contour plots showing phenotype and relative frequencies of DC2 subsets in the skin-dLN of naïve $\| / 4 \mathrm{ra}^{\mathrm{f} / \text { - }}$ or zDC-Cre $/ / 4 \mathrm{ra}^{\mathrm{f} /-}$ mice.

(E) Frequency of cytokine-expressing CD4 T cells in the skin-dLN cells of $1 / 4 \mathrm{ra}^{\mathrm{f} /-}$ or $/ 14 \mathrm{ra}{ }^{\mathrm{f} /-} \mathrm{zDC}-$ Cre mice 5 days after intradermal immunization with $\mathrm{Ms}, \mathrm{Nb}, \mathrm{Ca}$ or PBS as a control. Intracelluar cytokine staining was performed after 5h PMA+ionomycin stimulation. Symbols show mean \pm SEM and refer to 8-11 mice/group pooled from 2-3 independent experiments. $P$ values were determined using a two-way ANOVA with Sidak correction.

(F) Frequency of GATA3 and RORyt-expressing CD4 T cells in the skin-dLN cells of $1 / 4 \mathrm{ra}^{\mathrm{f} / \text { - or }}$ $\| 4 \mathrm{ra}^{\mathrm{f} /-} \mathrm{zDC}$-Cre male mice 5 days after intradermal immunization with $\mathrm{Nb}, \mathrm{Ca}$, or PBS as a control. Intracelluar transcription factor staining was without PMA+ionomycin stimulation. Symbols show mean \pm SEM and refer to 6-8 mice/group from 2 independent experiments. $P$ values were determined using two-way ANOVA with Sidak correction.

$(\mathbf{C}, \mathbf{E}, \mathbf{F})$ * refer to the comparisons between $\mathrm{C57}$ and STAT6-KO chimeras, \# refer to the comparison between PBS and immunized. ${ }^{*} p<0.05 ;{ }^{* *}, \# p<0.01 ; * * * \# \#<0.001$; only significant comparisons are indicated.

(G) Total number of AF488 cells in the skin-dLN of $\| 4 \mathrm{ra}^{\mathrm{f} /-}$ or $/ 14 \mathrm{ra}^{\mathrm{f} /-} \mathrm{zDC}$-Cre mice $48 \mathrm{~h}$ after intradermal immunization with AF488-labelled $\mathrm{Nb}$ ( $\mathrm{Nb}$-AF488). Bar graph shows mean \pm SEM, each bar refers to 7-9 mice pooled from 2 independent experiments. $P$ value was determined using a two-tailed Student's t-test.

(H) Representative contour plots showing $\mathrm{Nb}$-AF488 $8^{+}$uptake by DC2 subsets in the skin-dLN of $\| 4 \mathrm{ra}^{\mathrm{f} /-}$ or $\| 4 \mathrm{ra}^{\mathrm{f} /-} \mathrm{zDC}$-Cre mice 48 hours after intradermal immunization with $\mathrm{Nb}$-AF488.

(I) Pie charts showing the cellular composition of the AF488 ${ }^{+}$population in the skin-dLN of $\| 4 \mathrm{ra}^{\mathrm{f} /-}$ or $\| 4 \mathrm{ra}^{\mathrm{f} /-}$ zDC-Cre mice 48 hours after intradermal immunization with $\mathrm{Nb}$-AF488. Data refer to the experiments in (G).

(J) Histograms showing the expression of costimulatory molecules and Interferon-induced surface markers on $\mathrm{Nb}$-AF488 and $\mathrm{Nb}$-AF488 ${ }^{+}$DC2 populations from $/ 14 \mathrm{ra}^{\mathrm{f} /-}$ or $/ 14 \mathrm{ra}{ }^{\mathrm{f} /-}$ zDCCre mice 48 hours after intradermal immunization with $\mathrm{Nb}$-AF488. Data are concatenated from 3 mice in one of two independent experiments that gave similar results.

(K) Expression of selected T/r and Unc93b1 transcripts in DC2 subsets from the skin-dLN of naive C57BL/ 6 mice as determined by RNA sequencing. Bar graph shows mean $\pm S D$, each bar refers to 3 samples each comprising the pooled LNs of 3 mice. $P$ values were calculated by DESeq2; ${ }^{* *} \mathrm{p}<0.01 ;{ }^{* * *} \mathrm{p}<0.001$. 
A

C

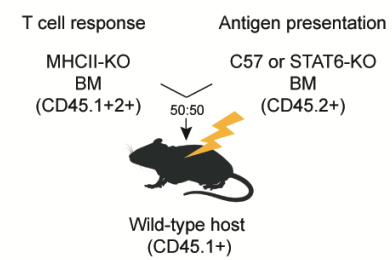

C57 chimera, Nb

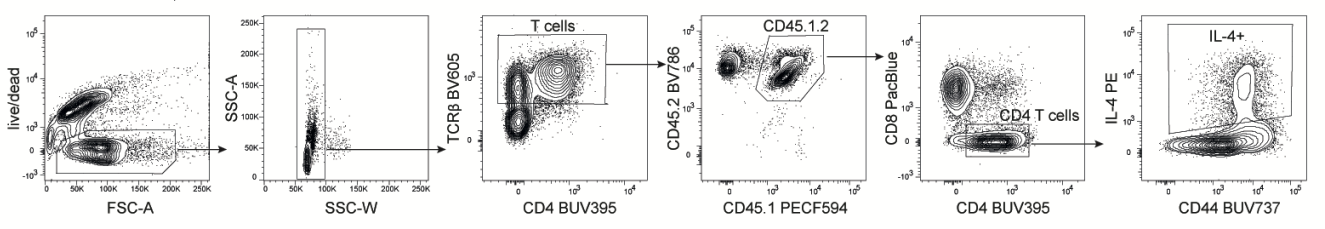

STAT6-KO chimera, $N b$

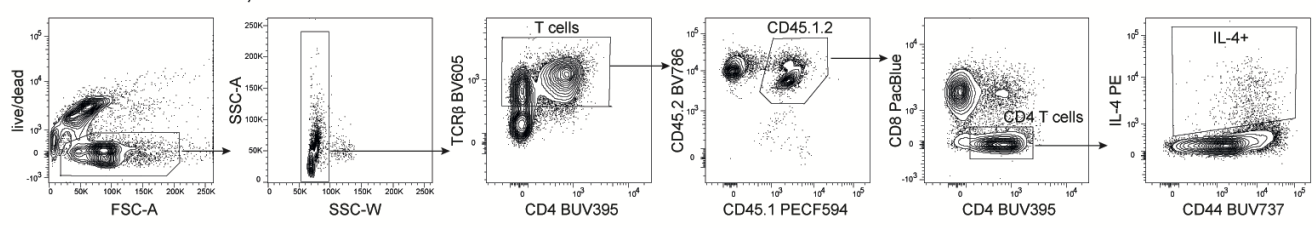

D

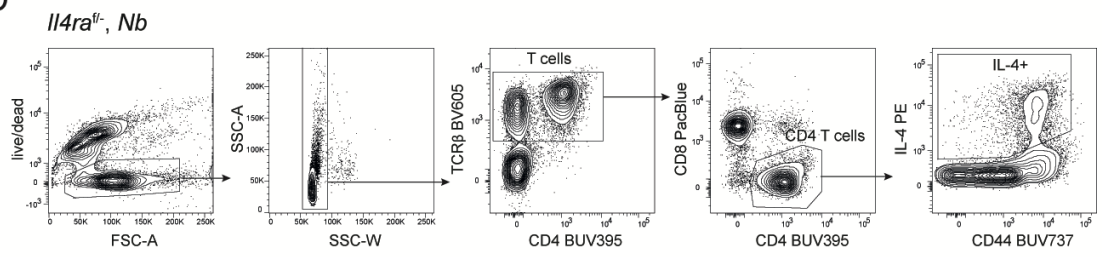

$\| 4 r^{a t}$ zDC-cre, $N b$

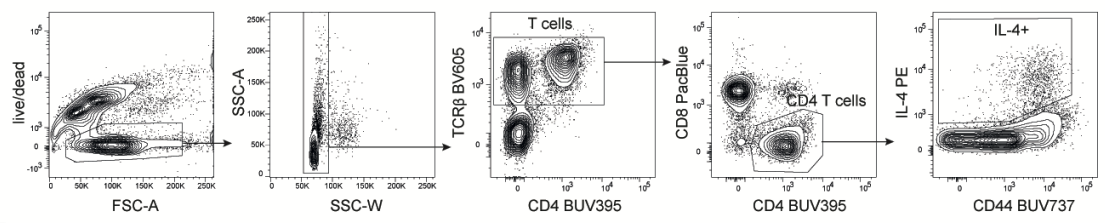

E

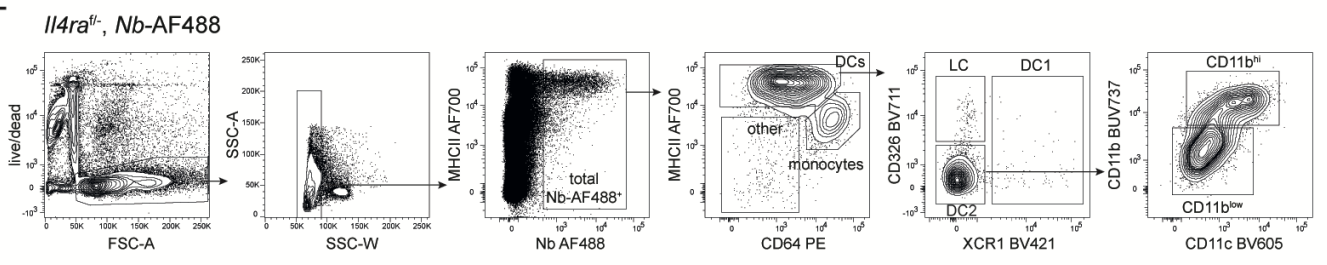

$14 r a^{f /-}$ zDC-cre, Nb-AF488
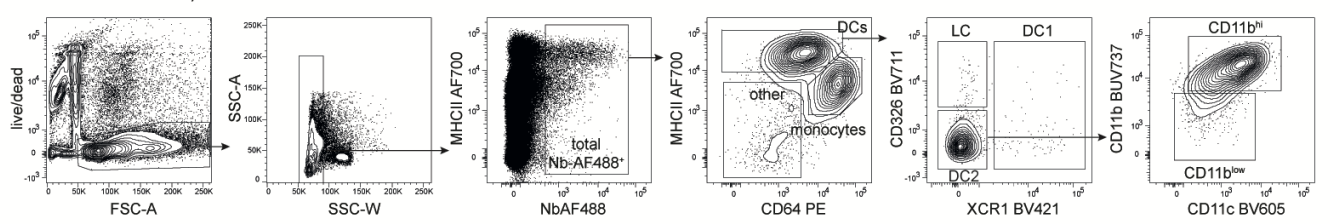

F

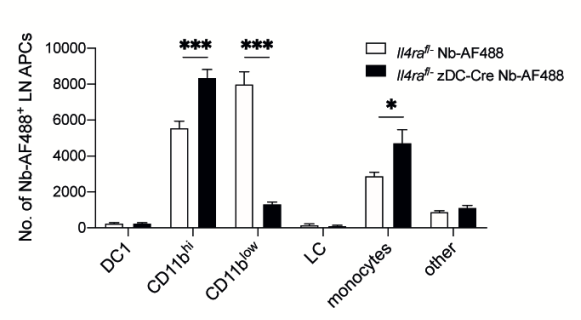

G

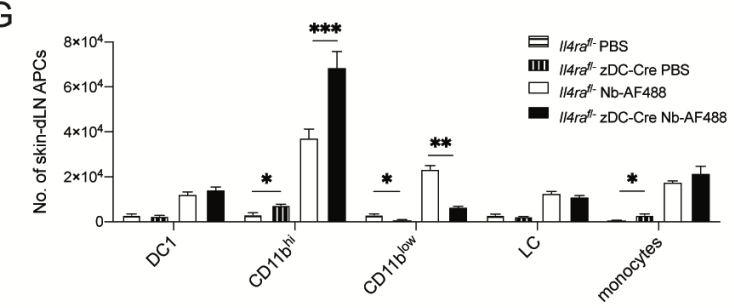

Figure S5. Relating to Figure 5: IL-13 signalling in DC2s is required for optimal IL-4 ${ }^{+}$Th responses in skin-draining lymph node (dLN). 
(A) Experimental set-up of mixed bone marrow (BM) chimeras in which the antigenpresenting function of $\mathrm{MHCII}^{+} \mathrm{CD} 45.2^{+}$conventional DCs from either C57 or STAT6-KO BM was assessed by measuring the response of STAT6-sufficient CD45.1 $1^{+} 2^{+} \mathrm{T}$ cells from MHCllKO BM.

(B) Relative frequencies of $\mathrm{MHClI}{ }^{+} \mathrm{CD} 45.2^{+}$and $\mathrm{MHCll}-\mathrm{KO} \mathrm{CD} 45.1^{+} 2^{+} \mathrm{DC} 2$ subsets in the skindLN of naïve C57 or STAT6-KO mixed BM chimeras. Bar graphs show mean \pm SEM, each bar refers to 8 mice pooled from 2 independent experiments. $P$ values were determined using a two-tailed Student's t-test. ${ }^{* *} \mathrm{p}<0.01 ;{ }^{* * *} \mathrm{p}<0.001$.

(C) Gating strategy for cytokine-positive CD45.1 $1^{+} 2^{+} \mathrm{CD} 4 \mathrm{~T}$ cells from the skin-dLN of $\mathrm{C57}$ or STAT6-KO mixed BM chimeras 5 days after intradermal immunization. Intracelluar cytokine staining was performed after 5h PMA+ionomycin stimulation. Gating for IL-4+ CD4 T cells after $\mathrm{Nb}$ immunization in C57 (upper panels) or STAT6-KO (lower panels) mixed BM chimeras is shown, other cytokines were gated in a similar manner.

(D) Gating strategy for cytokine-positive CD4 T cells from the skin-dLN of $/ / 4 \mathrm{ra}^{\mathrm{f} /-}$ or $/ 14 \mathrm{ra}^{\mathrm{f} /-}$ zDC-Cre mice 5 days after intradermal immunization. Intracelluar cytokine staining was performed after $5 \mathrm{~h}$ PMA+ionomycin stimulation. Gating for IL-4+ CD4 T cells after $\mathrm{Nb}$ immunization in $/ / 4 r a^{f /-}$ (upper panels) or $/ 14 r a^{f /-}$ zDC-Cre (lower panels) mice is shown, other cytokines were gated in a similar manner.

(E) Gating strategy for AF488 ${ }^{+}$cells in the skin-dLN of $/ 14 \mathrm{ra}^{\mathrm{f} /-}$ (upper panels) or $/ 14 \mathrm{ra}{ }^{\mathrm{f} /-} \mathrm{zDC}-\mathrm{Cre}$ (lower panels) mice 48 hours after intradermal injection of AF488-labelled $\mathrm{Nb}$ ( $\mathrm{Nb}$-AF488).

(F) Number of AF488 cells in the skin-dLN of $/ 14 \mathrm{ra}^{\mathrm{f} /-}$ or $/ 14 \mathrm{ra}^{\mathrm{f} /-}$ zDC-Cre mice 48 hours after intradermal injection of AF488-labelled $\mathrm{Nb}$. Bar graphs show mean $\pm \mathrm{SEM}$, each bar refers to 7 mice pooled from 2 independent experiments. $P$ values were determined using a twotailed Student's t-test. ${ }^{*} p<0.05 ; * * p<0.001$; only significant comparisons are indicated.

(G) Number of antigen-presenting cells (APCs) by population in the skin-dLN of $/ 14 \mathrm{ra}^{\mathrm{f} / \text { - or }}$ $1 / 4 \mathrm{ra}^{\mathrm{f} /-}$ zDC-Cre mice 48 hours after intradermal injection of AF488-labelled $\mathrm{Nb}$ ( $\mathrm{Nb}$-AF488) or PBS. Bar graphs show mean \pm SEM, each bar refers to 3-9 mice from 2 independent experiments. $P$ values were determined using a two-tailed Student's t-test to compare similarly treated groups of different genotype. ${ }^{*} p<0.05 ;{ }^{* *} p<0.01 ;{ }^{* *} p<0.001$; only significant comparisons are indicated. 

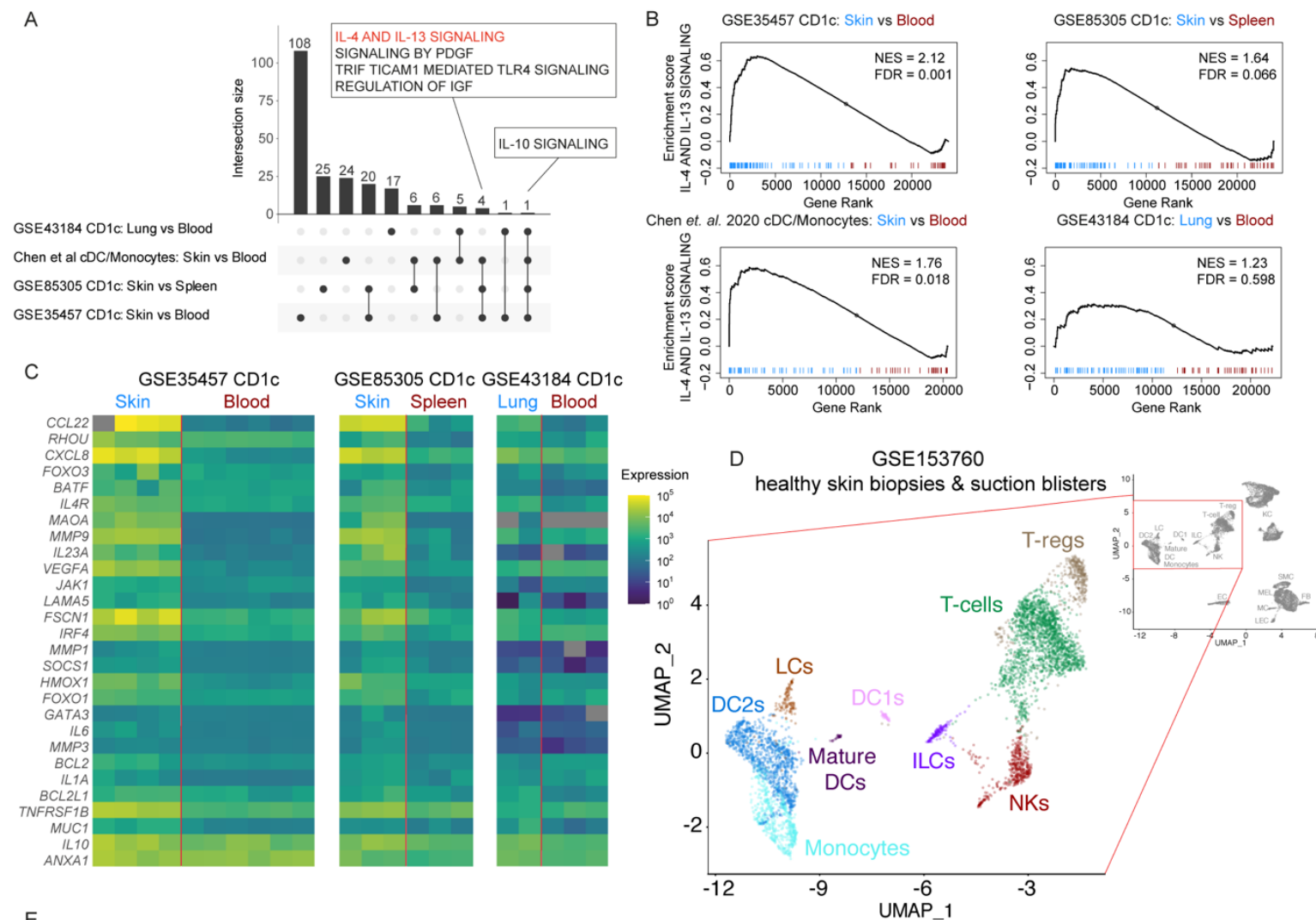

E
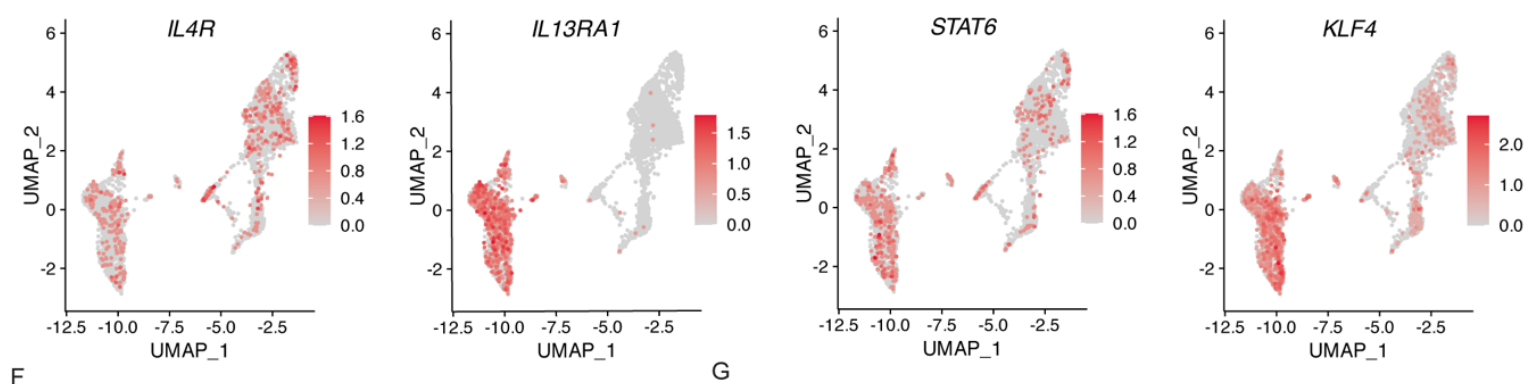

$\mathrm{F}$
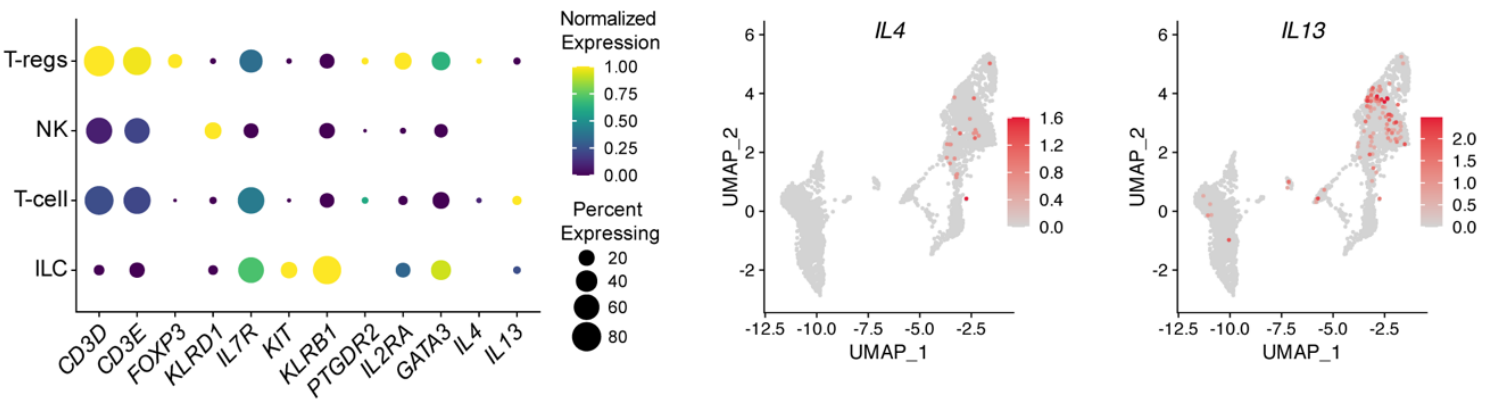

Figure 6. The transcriptome of DC2s from human skin, but not lung, is enriched in IL-4/IL13 signature genes.

(A) UpSet plot showing the numbers of Reactome pathways that are enriched in the transcriptome of sorted CD1c+ DC2s from lung vs blood (GSE43184), cDC/monocytes from from skin vs blood (Chen et al, 2020), CD1c+ DC2s from skin vs spleen (GSE85305) and CD1c+ DC2s from skin vs blood (GSE35457) as determined using Gene Set Enrichment 
Analysis (GSEA). All samples were from healthy donors. Pathways that are enriched only in skin samples (4 pathways) or in all comparisons (1 pathway) are listed.

(B) GSEA enrichment plots of IL-4 and IL-13 signaling pathway genes in human tissue DC2s compared to blood or spleen as described in (A).

(C) Heatmaps showing expression of GSEA core-enrichment genes of the IL-4 and IL-13 Reactome pathway in sorted CD1c+ DC2 from healthy human skin or lung compared to blood or spleen in the indicated microarray studies.

(D) Detail of a UMAP plot showing lymphocyte and myeloid cell clusters from scRNA-seq of skin biopsies and suction blisters of healthy controls from the published dataset GSE153760. DC1: conventional DC1s; DC2: conventional DC2s, ILC: Innate lymphoid cells; LCs: Langerhans cells; NK: Natural killer cells. The full UMAP plot in gray includes all the clusters and can be found in Fig S6B.

(E) Feature plots of the UMAP clusters in (D) showing the expression levels of IL4R, IL13RA1, STAT6 and KLF4 transcripts. Color intensity represents the level of normalized gene expression.

(F) Dot plot showing the expression of discriminatory markers for the different lymphocyte populations shown in (D).

(G) Feature plots of the UMAP clusters shown in (D) showing the expression levels of IL4 and IL13 transcripts. Color intensity represents the level of normalized gene expression. 
A
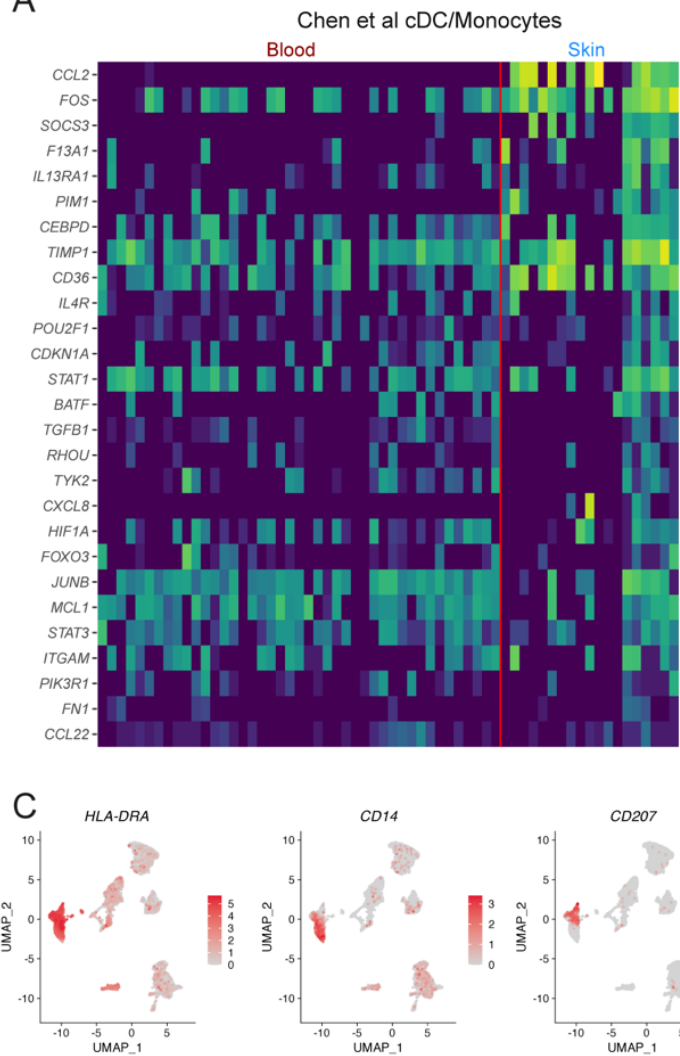

$C D 3 D$

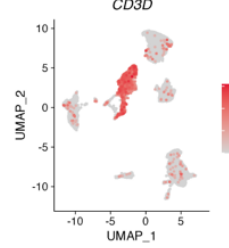

KRT5
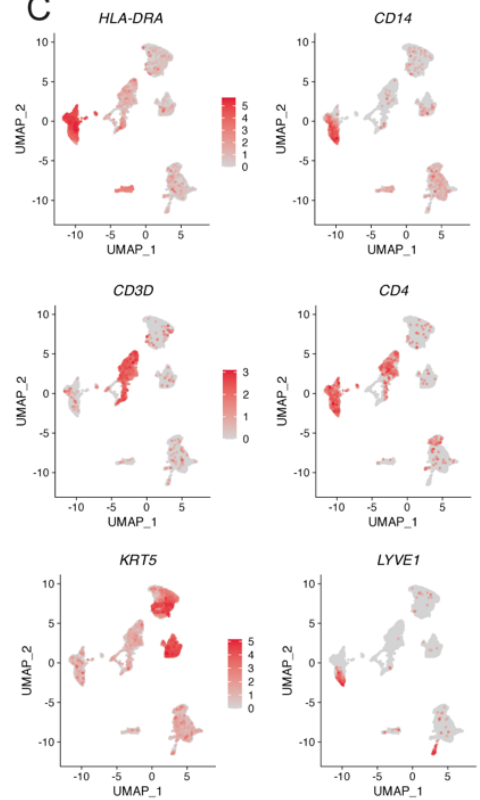

$C D 4$

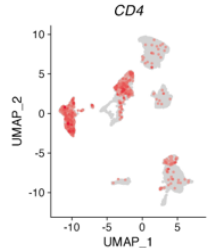

LYVE1
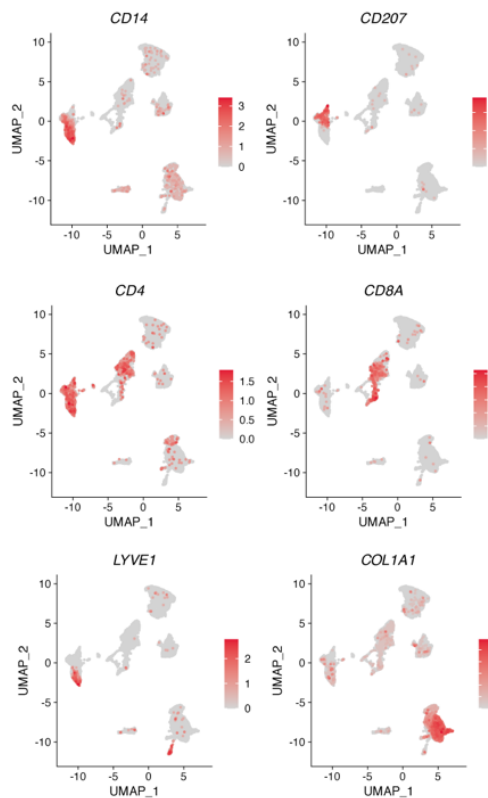

CD8A

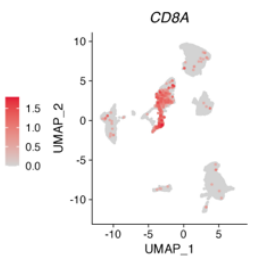

COLIA1
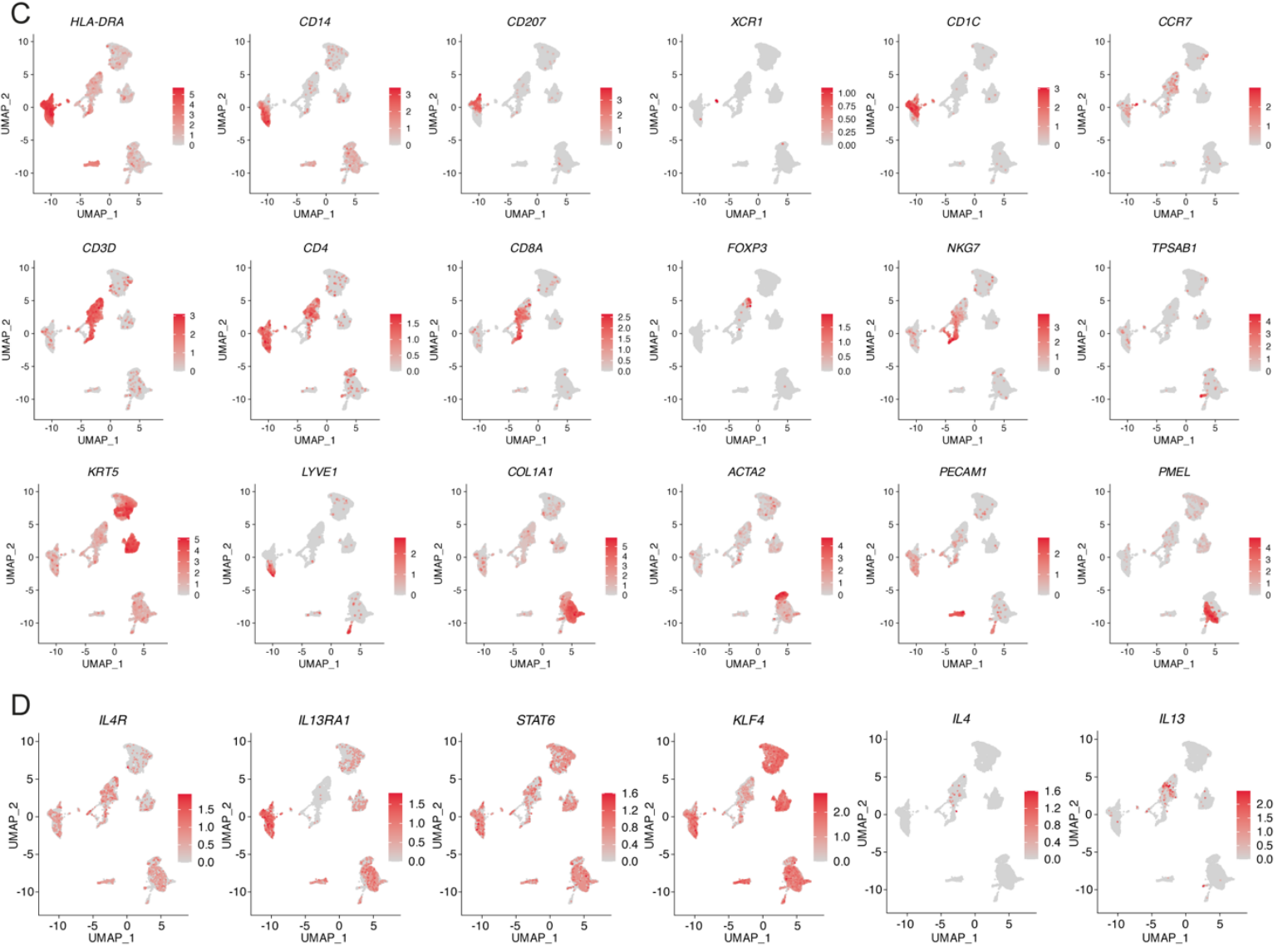

Figure S6. Relating to Figure 6: Enrichment of IL-4/IL-13 signature genes in DC2s from healthy human skin

(A) Heatmap showing the expression of GSEA core-enrichment genes of the IL-4 and IL-13 Reactome pathway in healthy control CDCs and monocytes from blood vs skin as determined by scRNAseq (Chen et al, 2020).

(B) UMAP plot showing ScRNA-seq subclusters of cells from skin biopsies and suction blisters of healthy controls from the published dataset GSE153760. EC: Endothelial cells, FB_1-2: 
Fibroblast clusters 1\&2, ILC: Innate lymphoid cells, LCs: Langerhans cells, LEC:

Lymphendothelial cells, MC: Mast cells, MEL: Melanocytes, NK: Natural killer cells, KC_1-7: Keratinocyte clusters 1-7, SMC: Smooth muscle cells, T-regs: Regulatory T cells.

(C) Feature plots of the UMAP clusters in B showing the expression level of cluster-specific transcripts used for cluster identification. Color intensity represents the level of normalized gene expression.

(D) Feature plots of the UMAP clusters in B showing the expression levels of IL4R, IL13RA1, STAT6, KLF4, IL4 and IL13 transcripts. Color intensity represents the level of normalized gene expression. 